


\title{
Functional neuroimaging of pathophysiological mesolimbic dopamine system and aberrant motivational salience in schizophrenia
}

\author{
Dissertation \\ zur Erlangung des mathematisch-naturwissenschaftlichen Doktorgrades \\ „Doctor rerum naturalium“ \\ der Georg-August-Universität Göttingen \\ im Promotionsprogramm ,Behavior and Cognition“ \\ der Georg-August University School of Science (GAUSS)
}

vorgelegt von

Anja Richter

aus Halle (Saale)

Göttingen, 2017 


\section{Betreuungsausschuss}

1. Betreuer: Prof. Dr. Oliver Gruber, Sektion für Experimentelle Psychopathologie und Bildgebung, Klinik für Allgemeine Psychiatrie, Universitätsklinikum Heidelberg

2. Betreuer: Dr. Igor Kagan, Kognitive Neurowissenschaften, Deutsches Primatenzentrum, Göttingen

3. Betreuer: Prof. Dr. Andreas Glöckner, Allgemeine Psychologie - Urteilen, Entscheiden, Handeln, FernUniversität in Hagen

\section{Mitglieder der Prüfungskommission}

Referent: Prof. Dr. Oliver Gruber, Sektion für Experimentelle Psychopathologie und Bildgebung, Klinik für Allgemeine Psychiatrie, Universitätsklinikum Heidelberg

Korreferent: Dr. Igor Kagan, Kognitive Neurowissenschaften, Deutsches Primatenzentrum, Göttingen

\section{Weitere Mitglieder der Prüfungskommission:}

Prof. Dr. Andreas Glöckner, Allgemeine Psychologie - Urteilen, Entscheiden, Handeln, FernUniversität in Hagen

PD Dr. Peter Dechent, MR Forschung in der Neurologie und Psychiatrie, Universitätsmedizin Göttingen

Dr. Roberto Goya-Maldonado, Labor für Systemische Neurowissenschaften und Bildgebung in der Psychiatrie, Klinik für Psychiatrie und Psychotherapie, Universitätsmedizin Göttingen

Prof. Dr. Annekathrin Schacht, Experimentelle Psycholinguistik, Courant Forschungszentrum Textstrukturen, Georg-August-Universität Göttingen

Tag der mündlichen Prüfung: 03.04.2017 


\section{Table of contents}

I Summary ................................................................................................................................... i

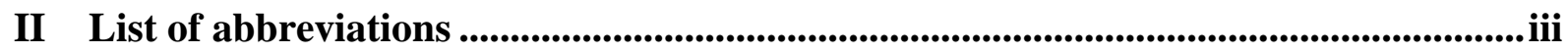

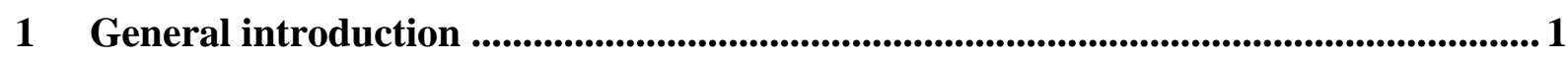

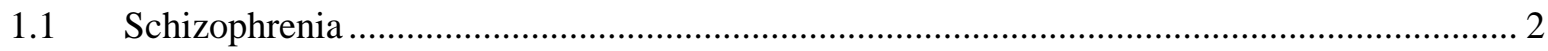

1.1.1 Symptoms, etiology, genetic and environmental risk factors ...................................................... 2

1.1.2 Pathophysiology: prevalent neurochemical hypotheses ........................................................... 4

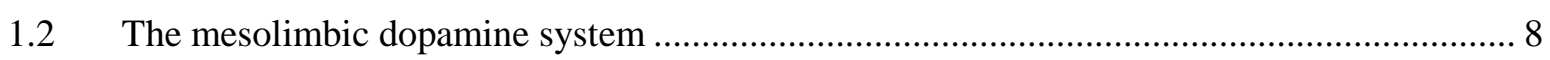

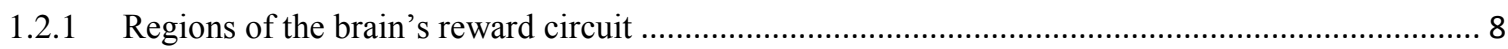

1.2.2 Neural mechanisms of reward processing and motivated behavior: evidence from animal and

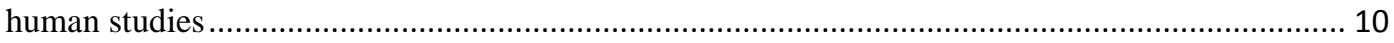

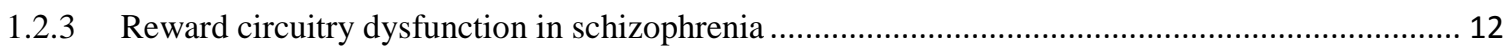

1.3 The concept of salience and the role of the mesolimbic dopamine system in processing salient events in general - Beyond the context of reward processing .................................. 13

1.4 Experimental methods used in the reported studies ......................................................... 15

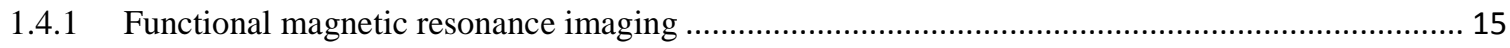

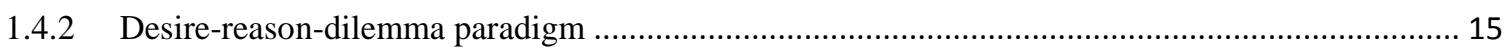

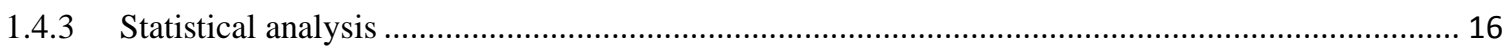

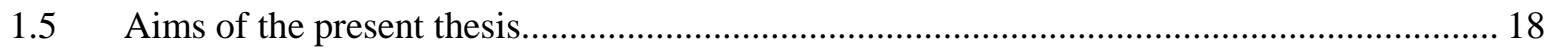

2 Hyperresponsivity and impaired prefrontal control of the mesolimbic reward system in schizophrenia ..................................................................................................21

2.1 Appendix: Supplementary data .......................................ehler! Textmarke nicht definiert.

3 Influence of ventral tegmental area input on cortico-subcortical networks underlying action control and decision making .............................................................. 23

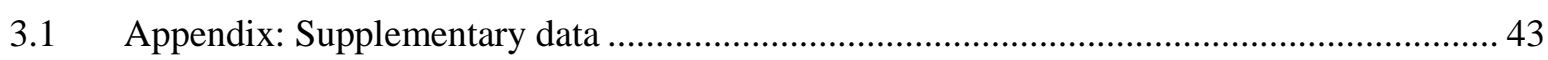

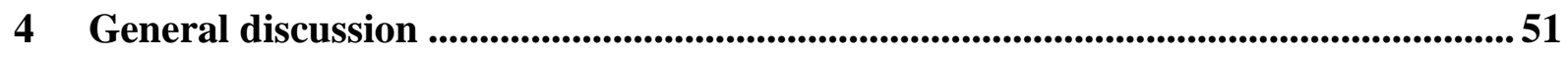

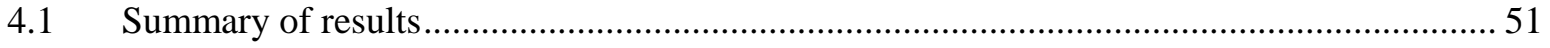

4.2 Observed findings of reward circuitry dysfunctions in schizophrenia in the present thesis

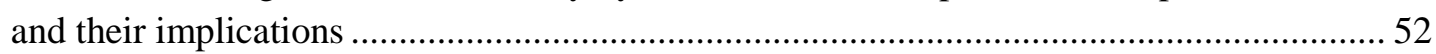

4.3 The mesolimbic dopamine system and its role in processing different forms of biologically

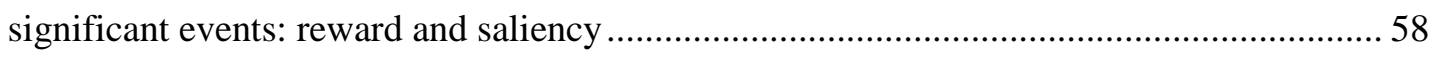

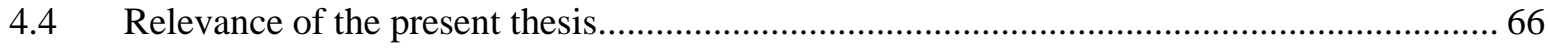




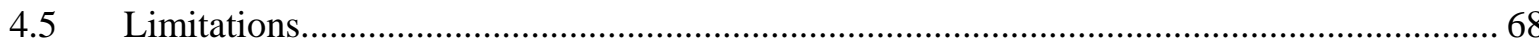

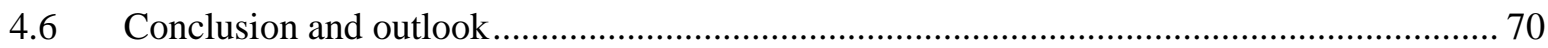

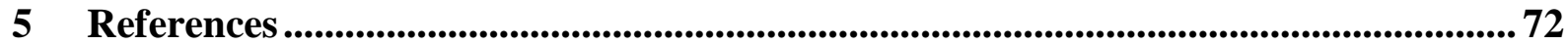

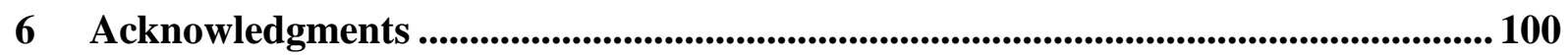




\section{Summary}

Schizophrenia is a common and devastating psychiatric illness with prominent variability regarding its process, symptomatology and treatment response. The disorder is characterized by three broad types of symptoms including positive symptoms such as hallucinations, delusions and disorganized thinking, negative symptoms involving blunted affect and anhedonia as well as cognitive impairments affecting working memory and attentional processes, learning and executive functions. Despite extensive research in the past, the etiology of the disorder remains still undetermined. It is hypothesized that its onset, progression and symptoms are influenced by an interaction of various susceptibility genes and environmental risk factors.

Several neurochemical models have been established to explain the emergence and development of the diverse symptoms in schizophrenia. Its pathophysiology involves dysregulation of multiple pathways with strong evidence for dysfunctional neurotransmitter systems involving dopamine, glutamate, gamma-aminobutyric acid (GABA) and choline. Thereby, dopaminergic dysfunction has been proven to play a major role in the pathogenesis of schizophrenia leading to detrimental consequences in reward processing and decision making. Previous studies provided evidence that dopamine metabolites were not elevated in the whole brain but rather that there is a regionally specific prefrontal hypodopaminergic state and a subcortical hyperdopaminergic state in schizophrenia. However, the question of how an increase in striatal dopamine synthesis and release capacity causes the symptoms in schizophrenia remains unresolved.

The first aim of the present thesis was to investigate how pathophysiological changes in patients with schizophrenia alter the functional activity and connectivity within the mesocorticolimbic dopamine system during reward-related decision making. In the previously introduced aberrant salience hypothesis it is postulated that in schizophrenic patients dysregulated dopamine transmission leads to an exaggerated release of dopamine and gives rise to the aberrant assignment of inappropriate salience and motivational significance to external objects and internal representations independent of the context. To account for this aspect, the second aim of the present thesis was to examine how the manipulation of salience through relative frequency of neutral and rewarding events affects the neural mechanisms and functional interactions of the mesolimbic dopamine system during decision making and action control. I addressed these questions by the use of functional magnetic resonance imaging (fMRI) and different versions of a reward-based decision making paradigm.

In the first study a group of 16 schizophrenic patients were matched to a group of 16 healthy control subjects regarding age, gender and years of education. All subjects had to follow specific 
task requirements to maximize their profit in the long run. In the desire context (DC), prior conditioned reward stimuli were allowed to obtain, whereas in the reason context (RC) these stimuli had to be rejected in favor of a superordinate long term goal. Compared to healthy controls, schizophrenic patients showed increased ventral striatal activation in response to the previously conditioned reward stimuli. Furthermore, they exhibited an attenuated suppression of reward signals in the ventral striatum (vStr) and ventral tegmental area (VTA) when they had to resist the rewards. This reduced suppression was associated with an impaired functional interaction between the vStr and both the anteroventral prefrontal cortex (avPFC) and ventromedial prefrontal cortex (VMPFC).

Overall, the increased bottom-up activation of the vStr may result from an intensified recruitment of this region during exaggerated assignment of salience to the conditioned rewards irrespective of the context. Moreover, the finding of disturbed cortico-striatal functional interaction in schizophrenic patients is in line with the dysconnection hypothesis of schizophrenia.

In the second study saliency was implemented by the experimental manipulation of both rewarding and neutral stimuli through relative frequency. Infrequent stimuli with long intervals between consecutive events are unexpected and hence more salient. Behavioral findings confirmed the successful experimental implementation of saliency by both increased error rates and reaction times in response to salient events. Moreover, infrequent neutral and goal-irrelevant events led to an increase of vStr and VTA activation and further cortical brain regions including the orbitofrontal cortex (OFC), inferior frontal gyrus (IFG) and anterior cingulate cortex (ACC). This demonstrates that the mesolimbic reward system was activated in response to saliency per se and provided direct evidence for the role of the mesolimbic dopamine system in processing salient events in general. An increase of activation in the vStr and VTA has been further demonstrated in response to infrequently presented rewards in situations where actions required a restraint from immediate rewards, indicating a boosting of activation in both brain regions, probably caused by the salient and rewarding attributes of the stimuli. This boosting of activation was accompanied by increased functional interactions between each other and further cortical brain regions such as the OFC, IFG and ACC.

Taken together, these subcortical and cortical brain regions working in collaboration may form a network to enable prioritized processing of salient stimulus attributes leading to adaptive and successful decision making.

Altogether, the reported findings addressed the main aims of the present thesis in extending the knowledge about how pathophysiological changes in schizophrenia affect behavior, functional activity and connectivity during reward processing and furthermore, how the manipulation of salience modulates neural mechanisms involved in action control and decision making. 


\section{List of abbreviations}

ACC

ANOVA

avPFC

BA

BOLD

CGI

CR

DC

DLPFC

DRD

DSM-IV

EPI

F

FDR

FEF

fMRI

FWE

GABA

GLM

hrf

ICD

IFG

IFJ

IFS

L

M

MADRS

MCC

mPFC

MFG

MNI

NAcc
Anterior cingulate cortex

Analysis of variance

Anteroventral prefrontal cortex

Brodmann area

Blood oxygenation level dependent

Clinical global impression

Conditioned reward

Desire context (study 1) and desire contrast (study 2), respectively

Dorsolateral prefrontal cortex

Desire-reason dilemma

Diagnostic and statistical manual of mental disorders

Echo-planar imaging

Female

False discovery rate

Frontal eye field

Functional magnetic resonance imaging

Family-wise error

Gamma-aminobutyric acid

General linear model

Hemodynamic response function

International statistical classification of disease

Inferior frontal gyrus

Inferior frontal junction

Inferior frontal sulcus

Left

Male

Montgomery-asberg depression rating scale

Middle cingulate cortex

Medial prefrontal cortex

Middle frontal gyurs

Montreal neurological institute

Nucleus accumbens 
NMDA $N$-methyl-D-aspartate

NT

Non-target (stimulus)

n.s.

Not significant

OFC

Orbitofrontal cortex

PANSS

Positive and negative syndrome scale

PCC

Posterior cingulate cortex

PCP

Phencyclidine

PET

Positron emission tomography

PFC

Prefrontal cortex

PPI

Psychophysiological interaction

$\mathrm{R}$

Right

$\mathrm{RC}$

Reason context (study 1) and reason contrast (study 2), respectively

ROI

Region of interest

$\mathrm{SC}$

Saliency contrast

$\mathrm{SCR}$

Salient conditioned reward

SD

Standard deviation

$\mathrm{SDC}$

Desire saliency contrast

SEM

Standard error of the mean

SFG

Superior frontal gyrus

SPECT

Single photon emission computerized tomography

SPM

Statistical parametric mapping

sRC

Reason saliency contrast

SVC

Small volume correction

$\mathrm{T}$

Target (stimulus)

TE

Echo time

TR

Repetition time

VMPFC

Ventromedial prefrontal cortex

VOI

Volume of interest

vStr

Ventral striatum

VTA

Ventral tegmental area 


\section{General introduction}

The ability to appropriately react to unexpected environmental changes and to integrate rewardand decision-related information provided by the environment is crucial for life time success and individual well-being. Disturbances of these functions may lead to detrimental consequences in general information processing and decision making. Schizophrenia is a severely disabling disorder associated with enormous clinical and socioeconomic impact, affecting essential cognitive processes such as learning, working memory and attention resulting in impaired work, self-care and interpersonal relationships. Considering that there is accumulating evidence demonstrating detrimental deficits in decision making and reward processing in schizophrenic patients, the major aim of this thesis is to investigate the neural correlates underlying these cognitive processes in a reward-based decision making task in patients with schizophrenia and healthy subjects using fMRI. Thereby, this thesis concentrates on the functioning of the mesocorticolimbic dopaminergic system in schizophrenia and in the healthy brain in order to investigate how pathophysiological changes in schizophrenic patients may alter the functional activity and connectivity during reward processing. One prevalent hypothesis in schizophrenia suggested disturbances in the attribution of salience and motivational significance to external objects and internal representations due to dysregulated dopamine system (Kapur, 2003). To address this assumption, I further examined the impact of different salient events on neural mechanisms and cortico-subcortical functional interactions underlying action control and decision making. By investigating the healthy brain and relevant pathomechanisms in schizophrenia, the findings of this thesis may contribute to a broader knowledge about the principles of this disorder, and further the development of more specific treatment approaches in the future.

In this general introduction I give an overview on the disorder schizophrenia, including symptoms as well as genetic and environmental risk factors contributing to the emergence of the disease, I summarize genetic and neuroimaging findings and present prevalent hypotheses and explanatory models. I then report on the functioning of the mesolimbic dopamine system, the brain regions forming the reward circuit and the neural correlates of reward processing. Afterwards, I provide a basic understanding of the term salience and delineate the neural mechanisms underlying saliency processing in general. Then, I give an overview about the experimental methods used in the present studies, before describing the major goals and hypotheses of this thesis. 


\subsection{Schizophrenia}

Schizophrenia is a complex and severe mental disorder with patients exhibiting a variety of symptoms and functional outcomes. About $1 \%$ of the worldwide population is affected by schizophrenia (Perälä et al., 2007) which as a disorder is characterized by prominent variability and different gradients regarding the influence of key variables like sex, migrant status, urbanicity and economic status on incidence, prevalence and mortality of this disorder (for review see McGrath et al., 2008). The illness predominantly emerges in late adolescence and early adulthood and mostly persists throughout the patient's lifetime (American Psychiatric Association, 1994). Schizophrenia is heterogeneous in nearly all aspects like symptomatology, treatment response, prognosis, outcome and stability of diagnosis (Jablensky, 2001). In recent years, schizophrenia has been considered as neurodevelopmental disorder, where a complex interaction between genes and environmental factors induce the symptoms of the disease. In the following section I give an overview about these factors with the attempt to provide an understanding of the determinants of the disorder and to introduce schizophrenia in a more useful and clear perspective to the reader. However, one major goal of the present thesis was to explicitly investigate the behavioral and neural correlates of reward-based decision making in schizophrenia.

\subsubsection{Symptoms, etiology, genetic and environmental risk factors}

Schizophrenia is characterized by three broad types of symptoms, namely positive symptoms, negative symptoms and cognitive impairments. Positive symptoms involve the loss of contact with reality, including hallucinations, delusions and disorganized thinking. Negative symptoms represent diminished or absent emotional and behavioral processes, such as blunted affect, social withdrawal, alogia, avolition and anhedonia (Crow, 1980; Andreasen et al., 1995; Kirkpatrick et al., 2006). In addition, cognitive impairments in schizophrenia involve deficits in working memory, attention, learning as well as executive dysfunctions and are detectable years before the onset of symptoms (Cannon et al., 2002; Barnett et al., 2012; Genzel et al., 2015). These impairments result in deteriorated functioning in work, school, parenting, self-care, independent living, interpersonal relationships and leisure time (American Psychiatric Association, 1994; Green, 1996).

Despite the intense research which has taken place in recent decades the etiology of schizophrenia remains unknown. Schizophrenia is a highly heritable (around $80 \%$ ) psychiatric disorder (Owen et al., 2003) and it was found that the phenotype expresses the combined influence and interaction of multiple genes (Stefansson et al., 2009; Greenwood et al., 2011; Ayalew et al., 2012). 
Moreover, current research suggests a network of genetic, environmental, behavioral and neural factors to be responsible for its development and course. Recent genetic association and genomewide association studies identified several risk genes of schizophrenia. Due to intense research in this field (see Schizophrenia Working Group of the Psychiatric Genomics Consortium, 2014), I present only a selection of the most prominent schizophrenia risk genes, such as catechol-omethyl transferase (COMT; Chen et al., 2004; Shifman et al., 2004), dystrobrevin-binding protein 1 (DTNBP1; Straub et al., 2002; Maher et al., 2010), neuregulin1 (NRG1; Stefansson et al., 2002; Munafò et al., 2008), disrupted in schizophrenia 1 protein (DISC1; Pletnikov et al., 2008; Schumacher et al., 2009) and zinc finger protein 804A (ZNF804A) whose risk variant was associated with reduced cortical gray matter thickness in several brain regions in schizophrenia (Kirov et al., 2005; O’Donovan and Owen, 2011; Voineskos et al., 2011). These schizophrenia susceptibility genes affect diverse regulatory and signaling pathways, e.g. dopaminergic, glutamatergic and GABAergic pathways, as well as specific neural mechanisms, like those involving synapses, gray matter development and neural plasticity, among others (for review see Karam et al., 2010; Bennett, 2011; Schizophrenia Working Group of the Psychiatric Genomics Consortium, 2014; Hall et al., 2015). Moreover, most cases of schizophrenia are thought to be the consequence of a synergistic interplay of various susceptibility genes and several environmental factors (for review see Harrison and Weinberger, 2005). Selected environmental factors associated with schizophrenia are urbanicity (Pedersen and Mortensen, 2001), prenatal infection, season of birth, drug abuse (McGrath and Murray, 2011) and obstetric complications with hypoxia (Sommer et al., 2010). In turn, this complex gene-environmental interplay influences onset and progression of the disease and may contain epigenetic alterations like DNA methylation and histone acetylation (Sananbenesi and Fischer, 2009; van Os et al., 2008).

Recent attempts for exploring the etiology of schizophrenia and other psychiatric disorders made use of an endophenotype-based approach. So called "endophenotypes" represents quantifiable brain measures or other biomarkers, for example cognitive functions which were thought to be intermediate between genotype and the disease, and segregates with disease in families (for review see Gottesman and Gould, 2003; Cannon and Keller, 2006; Kendler and Neale, 2010). Examples of prominent neurocognitive candidate endophenotypes in schizophrenia are working memory function (Goldman-Rakic, 1999; Perlstein et al., 2003), sensory motor gating (Braff et al., 2001; Braff and Freedman, 2002; Freedman, 2003) and oculomotor function (Calkins and Iacono, 2000; Lee and Williams, 2000; Braff and Freedman, 2002) to name but a few. Deficits in working memory have consistently been uncovered in patients with schizophrenia (Gur et al., 2007; Toulopoulou et al., 2010) as well as in unaffected family members (Barrantes-Vidal et al., 2007; Giakoumaki et al., 2011). These findings suggest that the observed deficits in healthy 
relatives may be caused by common risk variants for schizophrenia (Toulopoulou et al., 2007; Owens et al., 2011a, b; Goldberg et al., 2012).

Overall, genetic, epigenetic and environmental factors may lead to developmentally mediated alterations in neuroplasticity which appear in diverse neurotransmitter and circuit dysfunctions and impaired connectivity (for review see Haller et al., 2014).

\subsubsection{Pathophysiology: prevalent neurochemical hypotheses}

Evidence is accumulating to support specific structural brain measures as candidate endophenotypes. Enlargement of the ventricular system, specifically the lateral and third ventricles has frequently been demonstrated in schizophrenia (for a meta-analysis see Wright et al., 2000). Additionally, in large cooperative analyses it was found that schizophrenic patients compared to healthy controls have significantly smaller hippocampus, amygdala, thalamus and nucleus accumbens and significantly larger pallidum and lateral ventricle (Hajima et al., 2013; van Erp et al., 2016). Furthermore, gray matter reduction was associated with longer duration of illness and higher dose of antipsychotic treatment (Hajima et al., 2013). Reduced brain volume was already found in people at high genetic risk of developing schizophrenia (Lawrie et al., 1999). As cortical and subcortical brain alterations have also been detected in other disorders such as major depressive disorder (Schmaal et al., 2016a, b) and bipolar disorder (Hibar et al., 2016), functional imaging methods seemed to be more promising in detecting disorder-related alterations because of increased sensitivity and specificity. Different imaging techniques such as positron emission tomography (PET) and fMRI have been used to identify activity in specific brain regions and potentially dysfunctional neural circuitry while patients perform cognitive tasks. Using PET, abnormalities in cerebral blood flow and metabolic rate have been proven in frontal and temporal regions, thalamus and cerebellum when schizophrenic patients performing tasks involving memory, executive functions and sustained attention (Buchsbaum et al., 1996; Ragland et al., 1998). Additionally, fMRI findings demonstrated abnormalities in neural activity in frontal and parietal areas (executive function and working memory domain: Riehemann et al., 2001; Callicott et al., 2000; Walter et al., 2003), temporal cortex (language production and processing: Kircher et al., 2002) and the hippocampus (episodic memory domain: Leube et al., 2003), among others. Moreover, fMRI studies suggest that symptoms in schizophrenia result from disturbances in the interaction of different neural networks rather than localized dysfunctions in single brain regions. Reports of fMRI activation during reward processing in schizophrenia patients will be highlighted in a separate section of this introduction (see subsection 1.2.3 Reward circuitry dysfunction in schizophrenia). 
As mentioned earlier, schizophrenia pathophysiology involves dysregulation of multiple pathways with strong evidence to support roles for dopaminergic, glutamatergic, GABAergic and cholinergic neurotransmitter systems and their interactions (Benes, 2009; Karam et al., 2010; Gibbons et al., 2013). Furthermore, a variety of susceptibility factors for schizophrenia including DISC1, NRG1 and its receptor ErbB4, COMT and the brain-derived neurotrophic factor (BDNF) along with their related pathways interact closely with dopaminergic, glutamatergic and GABAergic neurotransmitter systems (Karam et al., 2010).

So far, it still remains an open debate about how these neurotransmitter systems and their interactions contribute to the emergence of psychotic and negative symptoms in schizophrenia. For the sake of clarity, in the following I focus on the most prominent neurochemical models of schizophrenia and give an overview of the most widely considered dopamine hypothesis, the aberrant salience hypothesis and the glutamate hypothesis.

Dopamine was the first neurotransmitter system suggested to be strongly involved in schizophrenia and dysfunctional dopaminergic mechanisms are supposed to be central to the disorder. First evidence came from Carlsson and Lindqvist (1963), who demonstrated that administration of antipsychotic drugs like chlorpromazine and haloperidol influenced the metabolism of dopamine in animals. Furthermore, it has been shown that amphetamine can induce psychotic symptoms by increasing synaptic monoamine levels (Bell, 1973). Finally, in the 1970s studies have provided evidence that antipsychotic drugs block dopamine receptors which, in turn, directly correlated with its clinical effectiveness to treat the psychosis (Seeman and Lee, 1975; Creese et al., 1976; Seeman et al., 1976; Matthysse, 1973; Snyder, 1976). New insights from postmortem, metabolite, imaging and animal data extend the knowledge that dopamine metabolites were not increased in the whole brain by demonstrating a regionally specific prefrontal hypodopaminergic state resulting in a subcortical hyperdopaminergic state in schizophrenia (for reviews see Davis et al., 1991; Howes and Kapur, 2009). Previous PET- and SPECT-studies have actually provided evidence for increased presynaptic striatal dopamine synthesis capacity in acutely psychotic patients (Hietala et al., 1995; Howes et al., 2009), heightened level of dopamine release (Abi-Dargham et al., 1998; Laruelle and Abi-Dargham, 1999; for a meta-analysis see Kestler et al., 2001) and increased baseline occupancy of D2 receptors, the receptor most widely expressed in the striatum (for a meta-analysis see Laruelle, 1998; Abi-Dargham et al., 2000). It was further hypothesized that excessive dopamine activity in mesolimbic dopamine neurons may lead to positive symptoms, whereas abnormally low prefrontal dopamine activity may cause negative symptoms in schizophrenic patients. Previous studies revealed a direct link between D1 receptor density in PFC and severity of negative symptoms in schizophrenia (Abi-Dargham et al., 2002; for review see Abi-Dargham and Moore, 
2003; Guo et al., 2003). The dopamine hypothesis of schizophrenia was further endorsed by genetic studies suggesting that multiple interacting dopaminergic polymorphisms may increase risk for schizophrenia (Talkowski et al., 2008). In addition, presynaptic striatal dopaminergic alterations have already been proven in first-degree relatives of schizophrenic patients (Huttunen et al., 2008). Moreover, interplay between genetic factors influencing dopamine function and environmental risk factors may result in dopaminergic dysfunction.

In summary, molecular imaging studies show the regionally specific dopamine distribution in schizophrenia and that dopamine D2 receptors continue to be the dominant key docking sites for all currently licensed antipsychotic drugs leading to improvement of symptoms. However, the question remains to be answered how overactivity of dopaminergic synapses in the mesolimbic system may produce the positive symptoms of schizophrenia and how low dopamine activity in prefrontal brain regions could lead to the emergence of negative symptoms, respectively.

Based on incentive salience models (for reviews see Berridge and Robinson, 1998; Berridge, 2007), Kapur (2003) provided a framework which attempts to link neurochemical dysfunction to clinical expression of positive symptoms in schizophrenia by using concepts of salience and reward. Usually, dopamine is released in response to specific stimuli, for example those previously related to a reward. This context-driven activity of the dopamine system mediates the experience of novelty and the acquisition and expression of appropriate motivational salience in response to the subject's experiences (for review see Shizgal, 1997; Berridge and Robinson, 1998). In contrast, it is proposed that in patients with schizophrenia dysregulated dopamine transmission leads to an elevated release of dopamine, independent of the context. This neurochemical disturbance usurps the normal process of stimulus- and context-driven salience attribution and leads to aberrant assignment of inappropriate salience and motivational significance to external objects and internal representations. Thus, the dopamine system which under normal conditions is a mediator of context-driven salience becomes a creator of aberrant novelty and salience in the psychotic state (see Kapur, 2003 for an overview). Already in prodromal patients undergoing aberrant salience in the absence of sustaining stimuli results in subtly altered experiences of the world without a clear reason or explanation for them. As a consequence thereof, it is hypothesized that delusions and hallucinations appear over time as the individual's own explanation of the experience of aberrant salience in an effort to make sense of them. In fact, these individually constructed delusions are inspired by the psychodynamic themes relevant to the patient and are influenced by their cultural context. This may explain how the same neurochemical dysregulation lead to variable clinical manifestations in different individuals and different cultures (Kapur, 2003; Kapur et al., 2005). Further data suggest that the aberrant attribution of salience may also be the reason for the emergence of negative symptoms. It is 
argued that due to the dopamine dysregulation in psychosis, it is possible that a reward-related stimulus fails to cause a sufficiently large phasic dopamine response. In turn, these misguided signals may decrease the value of motivationally salient stimuli (Roiser et al., 2009). Overall, dysregulated dopamine transmission may increase the noise in the system resulting in "drowning out" dopaminergic signals linked to stimuli which indicate reward (Grace, 1991; Roiser et al., 2009; Howes and Kapur, 2009). As a consequence of the caused reduced motivational drive, negative symptoms may appear over time such as blunted affect and anhedonia.

Although dopaminergic D2 receptor antagonists such as antipsychotic medication are mainly used to treat the symptoms of schizophrenia, accumulative evidence suggests that changes in multiple neurotransmitter systems, particularly the glutamate system, may be abnormal and underlie the symptoms of schizophrenia. A potential role for glutamatergic mechanisms in general and $\mathrm{N}$ methyl-D-aspartate (NMDA) receptors in particular was first proposed about 20 years ago. The also called NMDA receptor hypofunction hypothesis (Olney and Farber, 1995; Olney et al., 1999) based on the observation in which drugs such as phencyclidine (PCP) and ketamine, both noncompetitive antagonists of the NMDA receptor, are blocking neurotransmission at these receptors. This blocking immediately lead to psychotic symptoms and neurocognitive disturbances similar to those seen in schizophrenia, including hallucinations, thought disorder and negative symptoms (Krystal et al., 1994). Furthermore, a majority of susceptibility genes and environmental risk factors for schizophrenia appear to converge on the NMDA-mediated glutamatergic system and entail NMDA receptor hypofunction in the limbic system (for reviews see Collier and Li, 2003; Craddock et al., 2005; Harrison and Weinberger, 2005). Based on these findings, it has been assumed that glutamatergic disturbances may involve hypofunctioning of NMDA receptors on GABA interneurons in the thalamus and basal forebrain (Olney and Farber, 1995; Olney et al., 1999; Lindsley et al., 2006; Lisman et al., 2008). Activity and output in this corticolimbothalamic network is heavily suppressed and coordinated by local GABAergic interneurons. Impairment of these cells results in disinhibition of glutamatergic transmission throughout the circuit and to a subsequent cascade of excitotoxic events. This disruption of the functional integrity of the corticolimbic circuit may then result in negative symptoms and cognitive impairments (for review see Snyder and Gao, 2013).

Clinical challenge studies indicate that NMDA dysfunction may give rise to secondary dopaminergic dysregulation in striatal and prefrontal brain regions (see Javitt, 2007 for review). In fact, firing of dopaminergic neurons are regulated by glutamatergic inputs either directly or via GABAergic interneurons (Vollenweider et al., 2000; Aalto et al., 2005). Conversely, dopamine modulates glutamate projection neurons in the hippocampus and cortex (Hatzipetros and Yamamoto, 2006). It is hypothesized that hypoglutamatergic-mediated impairment of GABA 
interneurons could induce an enhanced dopaminergic activity in subcortical brain regions (Miller and Abercrombie, 1996). Yet, it remains an open debate whether abnormalities in neurotransmission of dopamine or glutamate are primary changes and lead to downstream effects at the respective other neurotransmitter system in the development of schizophrenia (see Stone et al., 2007 for review).

\subsection{The mesolimbic dopamine system}

The dopamine system is of special significance for humans and animals as it has been shown to play a significant role in several different aspects of brain function, including motor behavior, cognition and emotion. Moreover, converging evidence suggests a specific role for the mesolimbic reward system and its major dopaminergic input in coding rewards and rewardassociated events (e.g. Olds and Milner, 1954; Schultz et al., 1997; for review see Haber and Knutson, 2010). The evaluation of reward and punishment is central to the interaction with the environment. Reward is of major importance for driving incentive-based learning and for the development of adaptive goal-directed behavior, as this requires evaluation of rewards, associative learning and the ability to inhibit inappropriate choices in favor of developing appropriate actions (Haber and Knutson, 2010). The integration of reward-related information is faciliated by distinct cortical and subcortical brain regions forming the reward circuit (see O’Doherty, 2004 for review).

It has been shown that the dopamine system is the last monoamine system to be laid down in the rat brain during ontogeny (Lauder and Bloom, 1974), suggesting that this system may have a significant stabilizing and integrative influence on brain circuits. Pathological changes may destabilize several of these circuits in functionally important ways which may lead to a number of neurological and psychiatric conditions (Grace, 2016).

\subsubsection{Regions of the brain's reward circuit}

Olds and Milner (1954) have demonstrated that electrical stimulation of specific regions of rat brain produced positive reinforcement and rewarding experiences. Moreover, activation of dopaminergic neurons of the midbrain VTA by electrical stimulation of the medial forebrain bundle produces the most stable rewarding effects (Bozarth, 1994). During the past decades, considerable knowledge has been gained regarding the anatomical basis of these brain regions involved in distinct pathways. 
The neurotransmitter dopamine is released from neurons in the dopaminergic midbrain (substantia nigra pars compacta and VTA) which have widespread projections to brain regions known to be involved in reward processes and guiding goal-directed behavior (for reviews see Wise, 2004; Grace et al., 2007; Ikemoto, 2007; Sesack and Grace, 2010). Cells in the medial part of the VTA innervate most strongly the nucleus accumbens (NAcc), particularly the shell. Thereby, the VTA and the vStr, including the NAcc, possess central roles within the reward circuitry since the NAcc integrates richly excitatory, inhibitory and modulatory afferents from cortical and limbic systems which are under the modulatory influence of dopamine. The NAcc and numerous of its inputs are also involved in regulating the activity states of dopaminergic neurons in the VTA either directly or indirectly (Sesack and Grace, 2010). Besides, dopaminergic neurons of the VTA project also to other limbic-related regions including the septum, amygdala and hippocampus. Furthermore, they innervate the medial prefrontal cortex ( $\mathrm{mPFC}$ ), cingulate and perirhinal cortex, OFC, the insular cortex and the hypothalamus (see Kringelbach and Rolls, 2004 for review; Wise, 2004; McClure et al., 2004; O'Doherty, 2004) which are considered as key components that regulate the reward circuit. Together, these overlapping projections form a complex and interacting neural network and are collectively referred to as the mesocorticolimbic dopamine system mediating different aspects of reward processing. Each of these key regions contributes with its individual functions to the overall implementation of motivational behavior, e.g. by identifying and evaluating the actual reward (Kringelbach and Rolls, 2004). In promoting and selecting goal-directed behavior, the ventral hippocampus supplies the NAcc with contextual and spatial information, therewith functionally gating the information flow in this region. Additionally, the basolateral amygdala is involved in expression of emotion, in learned emotional behaviors and codes and conveys information regarding conditioned associations as well as stimulus arousal. The PFC provides an integrative guidance on goal-directed behavior by supplying executive control (O’Donnell and Grace, 1995; Ambroggi et al., 2008; Ishikawa et al., 2008; Ito et al., 2008; Sesack and Grace, 2010).

For the present work the key regions of the reward system, namely the vStr, VTA and the PFC as well as the projections between them are of particular importance (see Figure 1). 


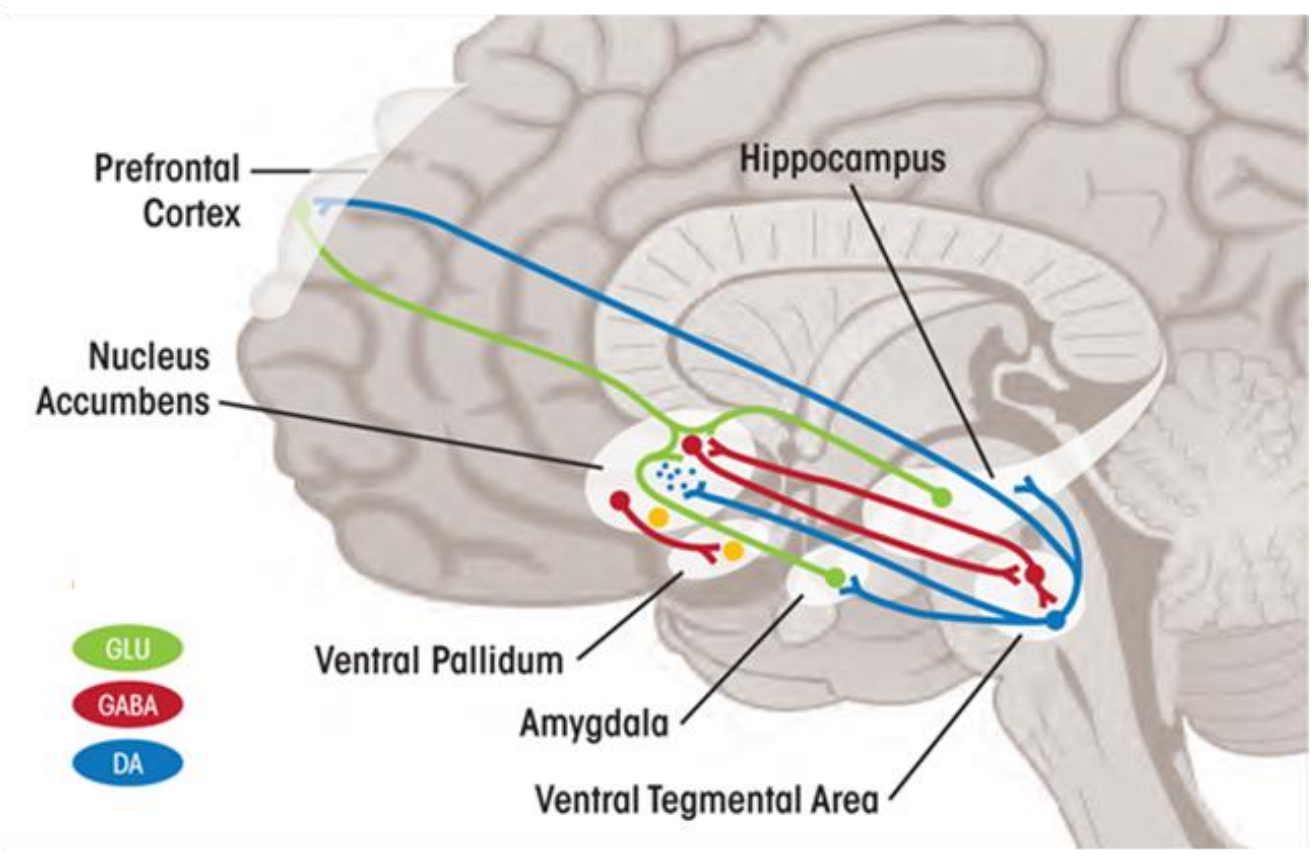

Figure 1. Brain regions of the mesocorticolimbic reward system.

Key regions of the reward circuitry and its dopaminergic, glutamatergic and GABAergic projections (figure is adapted with permission from Alim et al., 2012).

\subsubsection{Neural mechanisms of reward processing and motivated behavior: evidence from animal and human studies}

Reward processing can be divided into several phases including anticipation of rewards, reward outcome and reward-related learning which are mediated by the mesolimbic dopamine system.

In non-human primates, dopamine neurons in the substantia nigra and VTA have been shown to respond phasically to rewards and over time these neurons learn to fire in response to cues predicting rewards (Schultz et al., 1997). This firing pattern also applies to vStr neurons (Cromwell and Schultz, 2003) and prefrontal cortical regions, as shown by enhanced activation of these neurons during anticipation of reward (Watanabe, 1996; Schultz et al., 2000). Human studies using fMRI could replicate these findings by reports of increased neural responses in the striatum to cues that predict potential primary rewards including pleasant liquids (O'Doherty et al., 2002) or odors (Gottfried et al., 2002), and secondary rewards such as money (Knutson et al., 2001a, b). Like in animal studies, distinct contributions of subsections of the striatum in humans have been highlighted with the dorsal striatum (comprising nucleus caudatus and putamen) being activated when subjects pressed a button in response to a reward-predicting cue (O'Doherty et al., 2004). In contrast, the ventral striatum was activated during passively viewing responses to 
conditioned stimuli (Chumbley et al., 2014). This is in line with a reinforcement learning model, the so called actor-critic-model (Sutton and Barto, 1998) suggesting that the dorsal striatum may serve as an "actor" that preferable chooses actions associated with greater long-term reward, whereas the ventral striatum has the function of the "critic" that updates successive predictions of future reward and guides prospective reward receipt (O’Doherty et al., 2004).

Anticipated reward depends on distinct dimensions like magnitude, probability, uncertainty, delay and effort. It has been demonstrated that NAcc activation increases proportional to the magnitude of anticipated monetary reward (Knutson et al., 2001a). Thereby, a previous meta-analysis study has revealed that the NAcc was specifically activated during anticipation of monetary gains but not during anticipation of losses (Knutson and Greer, 2008). During reward anticipation, vStr activation increased with stimulus uncertainty (Cooper and Knutson, 2008). In a previous fMRI study of expected value, it has been shown that regions of the mesial PFC preferentially respond to rewarding outcomes (Knutson et al., 2003). Moreover, activation in this brain region correlated with both the anticipated magnitude and the anticipated probability of rewards (Knutson et al., 2005). Reward outcomes do also influence activation of the ventral striatum. Previous findings indicate that omission of expected reward led to a decrease of vStr activation (Berns et al., 2001). The observations of the striatum responding to events that predict rewarding outcomes support a prominent role for the striatum and its dopaminergic afferents in reward-based learning. Actually, it is assumed that midbrain dopamine neurons may track a reward prediction error, that is the difference between expected and obtained rewards (Schultz et al., 1997). In this hypothesis, the occurrence of an unexpected reward elicits an increase in phasic firing of dopamine neurons, generating a positive prediction error. In turn, when an expected reward fails to occur, dopaminergic firing is suppressed and a negative prediction error is recorded (Schultz et al., 1997). Human neuroimaging studies could support this assumption (e.g. Berns et al., 2001; Tobler et al., 2006). In fact, Abler et al. (2006) provided evidence that vStr activity reflects signaling of a reward prediction error implemented by reward probability. It was further assumed that the ventral striatum is involved in both Pavlovian and instrumental conditioning (O'Doherty et al., 2004). Furthermore, it has been shown that the vStr is activated when previously conditioned rewards were presented, reflecting an automatic bottom-up response (Diekhof and Gruber, 2010). In turn, the dorsal striatum has been reported to mediate the instrumental component of motivated behavior (O’Doherty, 2004; Elliott et al., 2004). 


\subsubsection{Reward circuitry dysfunction in schizophrenia}

Disturbances of the reward circuitry entail pathophysiological mechanisms that are common in distinct neurodevelopmental and psychiatric disorders. Reward-processing deficits have been proven in affective disorders, substance-use as well as obsessive-compulsive disorders, attention deficit/hyperactivity disorder and above all schizophrenia.

Internal representations of previous reward and motivational goals are used to drive current and future goal-directed behavior in a way to obtain desired outcomes. It has been shown that while the subjective experience of evoked positive emotions ("liking") is normal in individuals with schizophrenia, their ability to experience anticipatory pleasure ("wanting" component) and thus to set meaningful goals and initiate goal-seeking behavior is affected (for review see Barch and Dowd, 2010). This deficit in motivation and drive underlying impaired decision making represents a core symptom in schizophrenia. On the behavioral level, studies of reward learning have shown that sensitivity to reward is intact in schizophrenia. However, patients exhibited impairments in rapid reward learning based on trial-to-trial feedback, such as probabilistic reversal learning, during reward-related decision-making and also delay discounting (Waltz and Gold, 2007; Koch et al., 2010; Waltz et al., 2011; Avsar et al., 2013). These findings provide evidence that patients with schizophrenia have indeed intact hedonic responses but show overt motivational deficits and exhibit a reduced ability to pursue meaningful goals to reach desired outcomes (Barch and Dowd, 2010).

On the neural level, previous neuroimaging studies suggested reduced NAcc response to monetary-reward anticipation (Juckel et al., 2006a; Waltz et al., 2009) which was further correlated with the severity of negative symptoms in schizophrenic patients (Juckel et al., 2006b). This finding was extended by reports of already reduced vStr activation in response to rewards in healthy first-degree relatives of schizophrenia (Grimm et al., 2014). Besides, previous studies have shown suppressed brain activation in response to reward-predicting stimuli in unmedicated schizophrenic patients (Juckel et al., 2006a) as well as in patients with relatively high doses of typically antipsychotics that block dopamine D2 receptors in the striatum (Juckel et al., 2006b), whereas a lower dose of atypically neuroleptics restored activation of the ventral striatum in response to reward-predicting stimuli (Schlagenhauf et al., 2008). There is also evidence that the observed effects may be mediated by the certainty of rewards, since schizophrenic patients showed reduced vStr activity to unexpected rewards, but increased responses of midbrain and vStr to expected rewarding outcomes (Morris et al., 2012). Furthermore, a series of fMRI studies elucidated the neural correlates of learning dysfunction, demonstrating enhanced activation of the reward system in response to neutral stimuli during appetitive and aversive conditioning in schizophrenia (Romaniuk et al., 2010) and in individuals at risk for psychosis (Roiser et al., 
2013), as well as reduced brain activation for reward-associated prediction errors compared with neutral prediction errors (Murray et al., 2008). Overall, these observations highlight the complexity of response patterns to rewards in patients with schizophrenia.

\subsection{The concept of salience and the role of the mesolimbic dopamine system in processing salient events in general - Beyond the context of reward processing}

Animals and humans survival depends on the ability to detect and appropriately react to environmental changes, and in particular to preferentially process significant information. Thereby, significance processing underlies the motivation to maximize pleasure or reward and to minimize danger or threat. At first it involves early pre-attentive processing of sensory information (e.g. salience processing) and in a second step conscious goal-directed processing of salient input. Detecting unexpected, infrequent or novel events have been shown to cause a reflexive neural response in healthy subjects occurring pre-attentively (Kiehl et al., 2005). Even before a salient visual stimulus has been perceived in the fovea, midbrain dopamine neurons have been reported to already respond to such a stimulus (Redgrave et al., 1999). These neurons are activated by salient changes in the environment irrespective of whether the environmental change has relevance for the organism or not at the time the VTA dopamine neurons respond (Horvitz, 2002).

In the present thesis, the meaning of the term "salience" may be best characterized as remarkable feature of a stimulus that automatically captures the attention of an organism and involuntarily causes a switch in attentional and behavioral resources (Redgrave et al., 1999). Thereby, the salience of a given stimulus can either be context-dependent, influenced by behavioral context, or stimulus-inherent, depending on factors such as stimulus intensity, frequency of occurrence or novelty (Downar et al., 2002). In addition, higher order cognitive processes may also be involved in the processing of salient environmental changes (Horvitz, 2002). In a situation where salient as well as behaviorally relevant stimuli occur (e.g. when they are associated with a rewarding or punishing outcome), which require behavioral adjustment (e.g. a change in initial behavior to introduce approach or avoidance behavior) it is assumed that different attentional processes interact with each other. For example, infrequent behaviorally relevant events are salient due to their rarity and automatically provoke a bottom-up stimulus-driven orienting reflex. Additionally, when subjects are instructed to detect infrequent target stimuli to acquire an experimental goal, the achievement of this goal requires a voluntary adjustment of attentional and behavioral processes, or in other words a top-down processing (for review see Corbetta and Shulman, 2002). 
Rewards (e.g. money) are coincidently salient and behaviorally relevant to the organism as they are directly associated with the motivation to maximize reward and minimize punishment. This leads to the interruption of ongoing behavior and an adjustment of attentional resources in order to obtain the reward (Redgrave et al., 1999).

As already described in a former subsection of this thesis, numerous studies have proven that the mesolimbic system and its major dopaminergic input possess a specific role in reward processing, since dopamine neurons are phasically activated by reward, reward-predicting stimuli and code a reward-prediction error. The incentive salience hypothesis draws another line of evidence clarifying the role of dopamine in reward. In this theory, it is argued that the dopamine system is not needed either to mediate the hedonic impact of rewarding events or to mediate predictive associations involved in reward learning. It is further suggested that the process of reward is dissociable into a "wanting" and "liking" component, and that dopamine may mediate the "wanting" of rewards by attributing incentive salience with its perceptual as well as motivational features to a stimulus (Aosaki et al., 1995). According to the hypothesis, the brain's neural representations of a conditioned stimulus will be transformed by converting a stimulus from a neutral representation (only informative) into an attractive and "wanted" incentive that further can be attention-grabbing and may drive goal-directed behavior (Berridge and Robinson, 1998). In this way, dopamine modulates the motivational value of rewards in a manner separable from "liking" mechanisms and reward learning (for more information see Berridge and Robinson, 1998; Berridge, 2007).

Converging evidence suggests that dopamine-driven signaling may not be selective for rewards but instead may code all salient events in general, including and extending beyond rewards. Actually, midbrain dopamine neurons have been observed to respond to many different salient environmental events, including appetitive stimuli (e.g. Young et al., 1992; Mirenowicz and Schultz, 1996), but also aversive (e.g. foot shock: Young et al., 1993), infrequently presented stimuli as well as physically highly intense and novel stimuli without rewarding nature (Horvitz et al., 1997; Rebec, 1998; Downar et al., 2002). Otherwise, dopaminergic activation is suppressed by events that are associated with diminished arousal or reduced anticipatory excitement, including omission of expected rewards (Schultz et al., 1997, 1998; see Horvitz, 2000 for review). In addition, human striatal activations have been reported following punishment (Knutson et al., 2000; Knutson et al., 2003) and aversive stimuli (Becerra et al., 2001), neutral events when they are unexpected (Zink et al., 2003) as well as novel or infrequent events (Downar et al., 2002). Moreover, Zink et al. (2004) suggested that the role of the mesolimbic system in processing reward is dependent on the saliency modulated by the rewards receipt, rather than value or hedonic feelings. By coding all these events, signaling of mesolimbocortical and nigrostriatal dopamine neurons is hypothesized to promote attention towards important events in order that the 
organism is prepared to adequately react to these events, therewith influencing successful goaldirected behavior (Horvitz, 2000).

\subsection{Experimental methods used in the reported studies}

\subsubsection{Functional magnetic resonance imaging}

FMRI has become the most commonly used method for mapping brain function in humans and animals. Information processing in the brain is mediated by the release of neurotransmitter molecules which lead to changes in the membrane potentials of neurons. This neurotransmitter release is accompanied by metabolic changes in neurons and glia cells giving rise to an enhanced blood flow caused by neural activity leading to an increase in local blood oxygen (e.g. Logothetis et al., 2001; Matthews, 2002). As oxygenated and deoxygenated blood has been shown to exhibit different magnetic properties, the signal measured in fMRI depends on this change in oxygenation and is referred to as the blood oxygenation level dependent (BOLD) signal (Ogawa et al., 1990a, b). Furthermore, the hemodynamic response underlies the basic features of BOLD fMRI and determines how the data must be analyzed. The increase in blood flow that follows neuronal activity is relatively slow and reaches its maximum at about 5 seconds after stimulus onset. After that it rises within 1-2 seconds and returns to baseline by 12-20 seconds after stimulus onset (Hoge and Pike, 2002). It is assumed that the relationship between the neural response and the BOLD signal exhibits linear time invariant properties. For statistical analysis of fMRI data, a general linear model was used which relies critically on the assumption of linearity (Friston et al., 1994). This approach allows analyzing several variables in a linear combination, namely the time courses of activation in each voxel. A vector representing the temporal onset of stimulus presentation (for each stimulus type) was convolved with a canonical hemodynamic response function to create a predicted hemodynamic response to each experimental condition. Afterwards, linear t-contrasts were defined for assessing the specific effects of each condition of interest. For a more detailed review about the principles of fMRI see Huettel et al., 2009 and Poldrack et al., 2011.

\subsubsection{Desire-reason-dilemma paradigm}

In order to investigate neural correlates of reward and saliency processing in the two neuroimaging studies, I applied an event-related fMRI design with the pseudorandomized 
presentation of different stimulus types that allows for the analysis of transient changes in brain activation associated with discrete stimuli (e.g. infrequency effects which cannot be tested in a block-design). For this purpose, I made use of a monetary reward-based decision-making paradigm, the so called desire-reason-dilemma (DRD) paradigm. It has the advantage and was created in order to directly investigate reward-related brain activation depending on the interaction of bottom-up and top-down mechanisms, when previously conditioned rewards had to be accepted or rejected in favor of a superordinate long-term goal. It was further used to examine functional interactions between regions of the brain's reward circuit in situations where also impulse control is needed (Diekhof and Gruber, 2010; Diekhof et al., 2012a, b, c).

\subsubsection{Statistical analysis}

In order to investigate how pathophysiological changes in schizophrenia affect reward-related behavior, performance data of the first study were analyzed using repeated measures ANOVA with group (patients, controls) as between-subject factor and task-context (desire context (DC), reason context $(\mathrm{RC})$ ) as within-subject factor to examine how each group differ in correctly accepting and rejecting the bonus and target stimuli. In addition, in the second study behavioral data were analyzed using repeated measures ANOVA with the factors saliency (trials containing salient stimuli vs. trials containing non-salient stimuli), reward (reward stimuli vs. non-reward stimuli) and task-context (trials containing no target stimuli (desire) vs. trials containing target stimuli (reason)). This was done in order to examine how the experimental manipulation of salience influences performance and reaction times during reward-related decision making.

In both neuroimaging studies I applied a full factorial model to analyze random-effects on group level. Thereby, single-subject contrast images were taken to the second level to assess group effects. Factorial designs permitted testing of overall mean, main effects and interaction effects of all factors of interest (particular contrast, group (patients vs. controls) and saliency) in one analysis. This enabled me to test for specific differences between schizophrenic patients and healthy controls in brain activation modulated by different task context in the first study, and to examine the influence of salient rewarding and neutral events on functional activity in healthy subjects in the second study. A further convenience is that this approach is known to be robust to different sample sizes.

Moreover, I examined the functional connectivity between reward-related brain regions by performing psychophysiological interaction (PPI) analyses (developed by Friston et al., 1997). In particular, PPI analyses sought to identify functional interactions between the vStr and prefrontal brain regions in desire-reason-dilemma situations, when immediate reward contingencies and the 
superordinate task goal competed for action control. Previous studies have reported inhibitory influences of the avPFC on the vStr in healthy subjects using the DRD paradigm (Diekhof and Gruber, 2010; Diekhof et al., 2012a, b). Aims of the first study were to extend these findings by assessing the functional connectivity between these key regions in schizophrenic patients compared to healthy subjects. I assumed disturbed regulative influences of prefrontal brain regions on mesolimbic structures of the reward system in schizophrenic patients. Furthermore, aims of the second study were to modulate the functional connectivity within the reward system through the experimental manipulation of salience. I predicted an increased functional interaction between the VTA and other dopamine-targeted brain regions, since dopamine neurons of the VTA have been shown to predominantly code salience. First, neuronal activity in a seed region that has generated fMRI time courses was estimated. Local activation maxima in specific contrasts of interest were chosen as seed regions serving as physiological vector in the PPI analysis. Second, this estimate was multiplied by the task timing resulting in a prediction of neuronal activity associated with the experimental manipulation defined as specific task conditions in the paradigm serving as psychological vector. This new prediction was then convolved with the fMRI hemodynamic response to generate the predicted PPI BOLD time course. In turn, this time course went into the statistical analysis on single-subject-level to identify activation in other brain regions (Huettel et al., 2009). For more detailed information regarding the PPI analyses in the individual studies please see Chapter 2 and 3.

A critical issue for fMRI analysis is the multiple testing problem. Recently, a debate has arisen about the problem of false-positive findings in the field of neuroimaging, referring to studies using cluster-level inference approaches (Eklund et al., 2016). In the presented studies I reported the main results on a voxel-based-inference level which allows measuring whether there is a significant effect at each individual voxel. By doing this, it enables the observer to make very specific inferences and it was shown to be a more conservative approach in Statistical Parametric Mapping (SPM), the applied software package for analyzing fMRI data in the current studies (Wellcome Trust Centre for Neuroimaging, University College London, UK: http://www.fil.ion.ucl.ac.uk/spm/).

In order to correct for multiple testing, I applied several well reported and proven approaches to the fMRI data in this thesis (e.g. Huettel et al., 2009). Corrections for multiple comparisons were performed using family-wise error (FWE) and false-discovery rate (FDR) at $p<0.05$. In both cases, random field theory was used to estimate the number of independent statistical tests needed, based on the spatial correlation or smoothness of the experimental data (Worsley et al., 1996). Using random field theory, SPM determines the statistical threshold whose expected number of clusters/voxels of significant activation (expected Euler characteristic) corresponds to the alpha value of $p<0.05$. Furthermore, the minimum cluster size was set to be large enough to make it 
unlikely that a cluster of that size would occur by chance. Based on previous fMRI studies, the minimum cluster size was set to 10 voxels. For brain regions with a specific a priori hypothesis based on previous observations, namely the bilateral vStr, VTA and avPFC (Diekhof and Gruber, 2010; Diekhof et al., 2012a, b) small volume corrections were used. A sphere was placed around coordinates taken from previous studies where significant activations in these brain regions were found (e.g. Diekhof and Gruber, 2010). Hence, only a small proportion of voxels were tested which reduced the total number of statistical comparisons. Moreover, activations corrected for small volume are reported at a threshold of $\mathrm{p}<0.05$, FWE-corrected. For illustration purposes, I applied the more lenient criterion of $\mathrm{p}<0.05$, uncorrected, to figures in this thesis.

\subsection{Aims of the present thesis}

The ultimate goal of this study was to examine how pathophysiological changes of the mesocorticolimbic dopamine system in schizophrenia affect the behavior, functional activity and connectivity in a reward-based decision making task, and how the experimental manipulation of salience modulates neural mechanisms of as well as cortico-subcortical functional interactions within the reward circuitry.

In the previous sections, I have summarized several main neurochemical hypotheses and explanatory models assuming specific neurotransmitter systems as key players in the development of schizophrenia. Considering that the dopamine hypothesis in schizophrenia is the most widely accepted framework for explaining the emergence and development of this disorder, the central interest of the present thesis lied on the functioning of the mesocorticolimbic dopamine system in schizophrenia and healthy subjects.

To extend recent findings and to address the aberrant salience hypothesis in schizophrenia (Kapur, 2003) this thesis also focused on the role of the dopaminergic system in processing different forms of biologically significant stimuli, either rewarding and/or behavioral relevant or simply neutral and goal-irrelevant events. It is still not clear how saliency modulates the functional activity and connectivity within the reward circuitry. In order to address this question, I investigated VTA and vStr responses to different salient events in healthy subjects, with the purpose to apply this paradigm to patients with psychosis in the near future.

I made use of the fMRI technique and applied different versions of a reward-based decisionmaking paradigm allowing to systematically investigate reward-related activations and neural mechanisms underlying reward and saliency processing when I experimentally manipulated salience in the task. 
Chapter 2 reports a neuroimaging study where the aim was to investigate neural correlates of reward processing in patients with schizophrenia compared to a group of healthy control subjects. The main question was how schizophrenic patients differ in their functional activity and connectivity in response to previously conditioned reward stimuli in situations when the rewards had to be accepted or rejected depending on different task requirements. These experimental conditions implemented in the paradigm allowed the assessment of bottom-up activation of subcortical brain regions together with their top-down modulation by prefrontal regions when subjects had to resist the previously conditioned rewards to optimize their total outcome. On the basis of a series of previous schizophrenia studies, I expected significantly impaired rewardrelated behavioral responses in schizophrenic patients caused by disturbances in reward learning processes. I further hypothesized that patients with schizophrenia would show altered mesolimbic reward system activation to the reward stimuli in both experimental situations, and impaired topdown control mechanisms, probably due to disturbed influences of PFC to counteract rewardrelated activity in the dopaminergic reward system.

Based on the findings of the first study and to address the aberrant salience hypothesis in schizophrenia, in the study reported in Chapter 3 the impact of the modulation of salience on neural mechanisms and cortico-subcortical functional interactions involved in action control and decision making were examined. For this purpose, I created a modified version of the rewardbased decision making paradigm to systematically vary the salience of specific stimuli in the task. In particular, saliency was manipulated by altering the incidence of reward stimuli and taskirrelevant neutral stimuli. In addition to activations in response to salient rewarding and therefore goal-relevant events, I further investigated human mesolimbic responses to saliency per se without a reward association and behavioral relevance. The aim of this purpose was the modulation of the VTA input to the ventral striatum, since dopamine neurons of the VTA are well-known for coding saliency in general. Additionally, I intended to clarify whether the functional connectivity between subcortical brain regions belonging to the reward system and cortical regions increase or decrease by manipulating salience.

Based on previous studies, I hypothesized that the experimental factor saliency has an influence on behavioral data, in particular that behavioral responses to salient trials will be longer compared to non-salient trials. Moreover, I predicted greater activation of the mesolimbic reward system due to the salient relative to the non-salient events and that the functional connectivity within the reward system would be modulated by saliency.

In Chapter 4 I discuss the results in a broader context, draw conclusions and give future perspectives. 
Overall, the major goal of the present thesis was to contribute to the understanding about the emergence of schizophrenia by investigating the dopaminergic reward system, and to extend the knowledge about the functioning of the mesolimbic reward system in coding salient events in general and the underlying neural mechanisms in the human brain. 


\title{
2 Hyperresponsivity and impaired prefrontal control of the mesolimbic
} reward system in schizophrenia

\author{
Anja Richter ${ }^{\mathrm{a}}$, Aleksandra Petrovic ${ }^{\mathrm{a}}$, Esther K. Diekhof ${ }^{\mathrm{a}, \mathrm{b}}$, \\ Sarah Trost ${ }^{\mathrm{a}}$, Sarah Wolter ${ }^{\mathrm{a}}$, Oliver Gruber ${ }^{\mathrm{a}}$
}

${ }^{\mathrm{a} C e n t e r}$ for Translational Research in Systems Neuroscience and Psychiatry, University Medical Center Göttingen, 37075 Germany

a,b Biocenter Grindel and Zoological Museum, University of Hamburg, 20146 Germany

Journal of Psychiatric Research 71 (2015) 8-15

http://dx.doi.org/10.1016/j.jpsychires.2015.09.005 
Hyperresponsivity and impaired prefrontal control of the mesolimbic reward system in schizophrenia

Full published article can be found under the following link:

http://www.sciencedirect.com/science/article/pii/S0022395615002666 
Influence of ventral tegmental area input on cortico-subcortical networks underlying action control and decision making

\title{
3 Influence of ventral tegmental area input on cortico-subcortical networks underlying action control and decision making
}

\author{
Anja Richter ${ }^{\mathrm{a}, \mathrm{b}}$, Oliver Gruber ${ }^{\mathrm{a}, \mathrm{b}}$

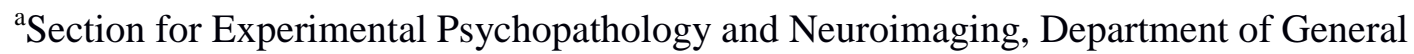 \\ Psychiatry, University of Heidelberg, 69115 Germany \\ ${ }^{\mathrm{b}}$ Center for Translational Research in Systems Neuroscience and Psychiatry, University Medical \\ Center Göttingen, 37075 Germany
}

Prepared for submission 


\begin{abstract}
It is argued that the mesolimbic system possess a more general function in processing all salient events, including and extending beyond rewards. Saliency was defined as an event that is unexpected due to its frequency of occurrence and elicits an attentional-behavioral switch. Using functional magnetic resonance imaging (fMRI), signals were measured in response to the modulation of salience of rewarding and non-rewarding events during a reward-based decision making task, the so called desire-reason dilemma paradigm (DRD). Replicating previous findings, both frequent and infrequent, and therefore salient, reward stimuli elicited reliable activation of the ventral tegmental area (VTA) and ventral striatum (vStr). When immediate reward desiring contradicted the superordinate task-goal, we found an increased activation of the VTA and vStr when the salient reward stimuli were presented compared to the non-salient reward stimuli, indicating a boosting of activation in these brain regions. Moreover, saliency per se without a reward association led to an increased activation of brain regions in the mesolimbic reward system as well as the orbitofrontal cortex (OFC), inferior frontal gyrus (IFG) and anterior cingulate cortex (ACC). Furthermore, we found a significantly increased functional connectivity between the VTA and vStr, confirming the boosting of activation in both brain regions. Finally, findings uncovered multiple increased functional interactions between cortical saliency-processing brain areas and the VTA and vStr underlying detection and processing of salient events and adaptive decision making.
\end{abstract}




\section{Introduction}

Converging evidence suggests a specific role for the mesolimbic reward system and its major dopaminergic input in coding rewards and reward-associated stimuli (Schultz et al., 1998; Schultz et al., 2000). However, it is argued that dopamine-driven signaling may not be selective for rewards, but instead may code all salient events in general. It has been reported that dopamine neurons in the substantia nigra and VTA respond to many different salient environmental events. Human striatal activations have been observed following punishment (Knutson et al., 2000; Knutson et al., 2003) and aversive stimuli (Becerra et al., 2001), neutral events when they are unexpected (Zink et al., 2003) as well as novel or infrequent events (Downar et al., 2002). Moreover, Zink et al. (2004) provided evidence that the mesolimbic system's role in processing reward is dependent on the saliency modulated by the rewards receipt, rather than value or hedonic feelings. Furthermore, it is hypothesized that dopamine becomes a mediator of incentive salience (Berridge and Robinson, 1998; Berridge, 2007) in a way that dopamine signaling may promote attention towards important events in the environment leading to an adjustment of behavioral reactions and therewith influencing goal-directed behavior (Horvitz, 2000).

In this study, the term salience is characterized as remarkable feature of an event that automatically captures the attention of an organism and involuntarily leads to a switch in attentional and behavioral resources (Redgrave et al., 1999). The salience of a given stimulus can either be context-dependent or stimulus-inherent, depending on factors such as frequency of occurrence or novelty (Downar et al., 2002). Thereby, infrequent behaviorally relevant events are salient due to their rarity and automatically provoke a bottom-up stimulus-driven orienting reflex. Furthermore, these behaviorally relevant events require an adjustment of both attentional and behavioral resources to obtain a goal, involving higher order cognitive processes (Corbetta and Shulman, 2002).

The current experiment sought to differentiate human mesolimbic dopamine responses to various salient events. The vStr as key region within the reward circuitry, integrates widespread limbic and cortical inputs, which are in turn under modulatory influence of dopaminergic neurons in the VTA (Haber and Knutson, 2010; Sesack and Grace, 2010). We used fMRI to examine the influence of the modulation of salience on neural mechanisms and cortico-subcortical functional interactions involved in action control of reward-based decision making. For this purpose, we created a modified version of the "desire-reason-dilemma" (DRD) paradigm (Diekhof and Gruber, 2010). This task allowed us to systematically investigate reward-related brain activations resulting from dopaminergic bottom-up mechanisms when previously conditioned reward stimuli had to be collected as well as top-down regulatory mechanisms when these reward stimuli had to be 
Influence of ventral tegmental area input on cortico-subcortical networks underlying action control and decision making

rejected in favor of a superordinate long-term goal. Saliency was manipulated by altering the frequency of occurrence of the reward stimuli. Additionally, we also wanted to investigate human mesolimbic responses to saliency per se without a reward association by manipulating the frequency of occurrence of goal-irrelevant non-rewarding stimuli. In this context, less frequent events are more salient because they are less predictable.

We hypothesized greater activation in the mesolimbic reward system due to the infrequent (high salience) relative to frequent (low salience) stimuli and that the functional connectivity within the reward system would be modulated by saliency.

\section{Materials and Methods}

\section{Subjects}

Twenty-six right-handed, healthy subjects (14 females), ages 20-35 years (mean: 25.27 years, SD: 4.21 years) were recruited from an academic environment. Subjects had normal or corrected-tonormal vision and no history of neurological or psychiatric disorders. Further exclusion criteria were lifetime diagnosis of substance dependence, substance abuse during the last month and cannabis abuse during the last two weeks. Ethical approval from local ethics committee and written informed consent were acquired before investigation. Subjects were paid for participation.

\section{Experimental task}

One day before the fMRI measurement, subjects underwent an operant conditioning task and a training session of the DRD paradigm. In the operant conditioning, nine differently colored squares were presented in a shuffled mode. Each trial consisted of two simultaneously presented colors. By free button choice, subjects were encouraged to explore which of these two presented colors were associated with an immediate reward to maximize their overall outcome by selecting one out of the two colors. Pressing button 1 meant that the left color was selected, whereas button 2 meant that the right color was selected. Two of the nine colors (red and green) always led to an immediate reward of 10 bonus points. Already in the operant conditioning task, red was presented six times less than the green squares. Decisions were immediately followed by a feedback indicating whether the decision for the left or right color led to an immediate reward or not. The overall goal of this procedure was to establish stimulus-response-reward contingencies, relevant for the next phase of the experiment. 
Influence of ventral tegmental area input on cortico-subcortical networks underlying action control and decision making

During this second phase, subjects underwent fMRI while performing a modified version of the DRD paradigm (see also Diekhof and Gruber, 2010). Stimuli were the same as in the operant conditioning phase, but now subjects had to pursue a superordinate long-term goal during task blocks of eight trials to acquire 50 points at the end of each block (see Figure 1). The superordinate task goal of an individual block was indicated by a cue showing the two target colors that had to be selected every time they occurred. When a target stimulus and a conditioned reward stimulus (CR) were presented simultaneously (dilemma situation), subjects always had to select the target stimulus. Otherwise they lost the 50 points and were only able to collect bonus points. In addition, subjects were allowed to select the CR for an immediate bonus, when it was presented together with a non-target stimulus (desire situation). These bonus points were added to the 50 points at the end of each block, if the long-term goal was successfully reached.

Seven of the nine colored squares occurred with same frequency during the whole experiment. The remaining two colors (red and yellow) were presented infrequently. Red was previously conditioned as a reward, served as salient conditioned reward stimulus (sCR) and occurred six times less than the non-salient conditioned reward stimulus (green; CR). Additionally, yellow served as salient control condition for the SCR and was presented six times less than the blue colored square, serving as control condition for the CR. Both yellow and blue were never presented as target stimuli, were never presented simultaneously with a sCR and CR respectively and were always shown as non-target. These stimuli were introduced to create an experimental baseline condition for the subsequent subtraction contrasts and to investigate brain responses to saliency per se without a reward association. Stimuli properties, including location, exact timing as well as preceding and following trials were pseudorandomized and counterbalanced in each run so that the sole manipulation of salience resulted from relative frequency. For more information, see Supplementary data.

\section{Behavioral data analysis}

Behavioral data were analyzed using the software package SPSS (IBM SPSS statistics 24.0). A repeated-measures ANOVA was performed with the 3 factors saliency (trials containing salient stimuli vs. non-salient stimuli), reward (reward stimuli vs. non-reward stimuli), and context (desire vs. reason (trials containing target stimuli)). Error and omission trials were excluded. Normal distribution of performance and reaction time data was tested using the KolmogorovSmirnov test. Differences between individual experimental conditions were subsequently assessed with a Bonferroni post hoc t-test, correcting for multiple comparisons. 


\section{FMRI data acquisition and analysis}

The experiment was performed on a 3 Tesla MRI scanner (Magnetom TIM Trio; Siemens Healthcare, Erlangen, Germany). Thirty-four axial slices parallel to the anterior-posterior commissure were acquired in ascending acquisition order (slice thickness $=3 \mathrm{~mm}$; gap $20 \%$ ) using a T2*-sensitive echo-planar imaging (EPI) sequence (interscan interval $1800 \mathrm{~ms}$; echo time 30 ms; flip angle $70^{\circ}$; field-of-view $192 \mathrm{~mm}$ ). A total of 1527 image volumes were acquired over the course of three functional runs. In the scanner, subjects saw the stimuli through goggles (Resonance Technology, Nothridge, USA) and responded via button presses on a fiber optic computer response device (Current Designs, Philadelphia, USA). Generation of stimuli and triggering of visual stimulation was achieved using the Presentation ${ }^{\circledR}$ Software (Neurobehavioral Systems, Albany, USA). Functional images were preprocessed and analyzed with SPM 8 (Wellcome Trust Centre for Neuroimaging, University College London, London, UK). At single subject level, each experimental condition was convolved with the hemodynamic response function to form regressors for each individual trial type: trials where a non-target was paired with a non-target, non-target paired with a target, CR paired with a non-target and CR paired with a target, each both for the non-salient trials and for the salient trials. The block cues indicating the target stimuli and the block feedback were also modeled as independent regressors, resulting in a total of 10 regressors. Linear t-contrasts were defined for assessing the specific effects of each condition of interest. Single-subject contrast images were taken to the second level to assess group effects with random-effects analyses. Group effects were examined using a full factorial model with the factors saliency (salient trials versus non-salient trials) and experimental trial type (trials combining non-target plus non-target stimulus; non-target plus target; CR plus non-target; CR plus target). For detailed information regarding calculated contrasts see SI.

Statistical effects were determined at a search criterion of $\mathrm{p}<0.005$, uncorrected, with a minimum cluster size of 10 voxels, if not otherwise indicated. Corrections for multiple comparisons were performed using family-wise error $(\mathrm{FWE})$ at $\mathrm{p}<0.05$. For brain regions with $a$ priori hypotheses i.e. for the bilateral VTA and vStr (VTA: \pm 8 -16 -16; 8 mm sphere; vStr: \pm 12 12 -4; 6 mm sphere, coordinates taken from Diekhof and Gruber, 2010) we used small volume corrections (SVC). To illustrate the magnitude of change due to the influence of salience, means of parameter estimate values for the desire and reason contrast were plotted. For this purpose, the MarsBar software (Brett et al., 2002) was used to extract each region of interests (ROIs) mean blood oxygenation level-dependent (BOLD) beta value with a sphere of $6 \mathrm{~mm}$ around the reported peak levels for each participant (see Figures 2 and 3). 


\section{Psychophysiological interaction analysis}

We assessed the functional interaction between the VTA and vStr to reveal the impact of saliency processing on the reward system by performing psychophysiological interaction (PPI) analyses (Friston et al., 1997). As seed regions, individual BOLD signal time courses were extracted from first eigenvariate time series (VOI; sphere of $8 \mathrm{~mm}$ ) of the local activation maxima within the right VTA (MNI coordinates: 9 -16 -17) and left vStr (MNI coordinates: -9 -8), which were the second-level local activation maxima in response to the salient non-target stimuli in the saliency contrast (see Table IV: saliency contrast: salient non-target vs. non-target > non-target vs. nontarget). Because we found increased activations of the bilateral VTA and vStr in the desire saliency contrast (sDC) and reason saliency contrast (sRC), first, we conducted a PPI analysis where the psychological vector consisted of the comparison between the SDC with the DC and second, where the psychological vector consisted of the comparison between the sRC with the $\mathrm{RC}$.

Furthermore, to examine functional interactions between further saliency-processing brain regions, VOIs of the second-level local activation maxima within the left OFC (MNI coordinates: -54 29 -5), left IFG (MNI coordinates: -54 29 1) and bilateral ACC (MNI coordinates: -18 11 25; 12828 ) in response to the salient non-target stimuli in the saliency contrast were extracted (see also Table IV). Based on these four seed regions, we also calculated two PPI analyses in the contrasts comparing the sDC with the DC and comparing the sRC with the RC. For more information, see SI. 
Influence of ventral tegmental area input on cortico-subcortical networks underlying action control and decision making

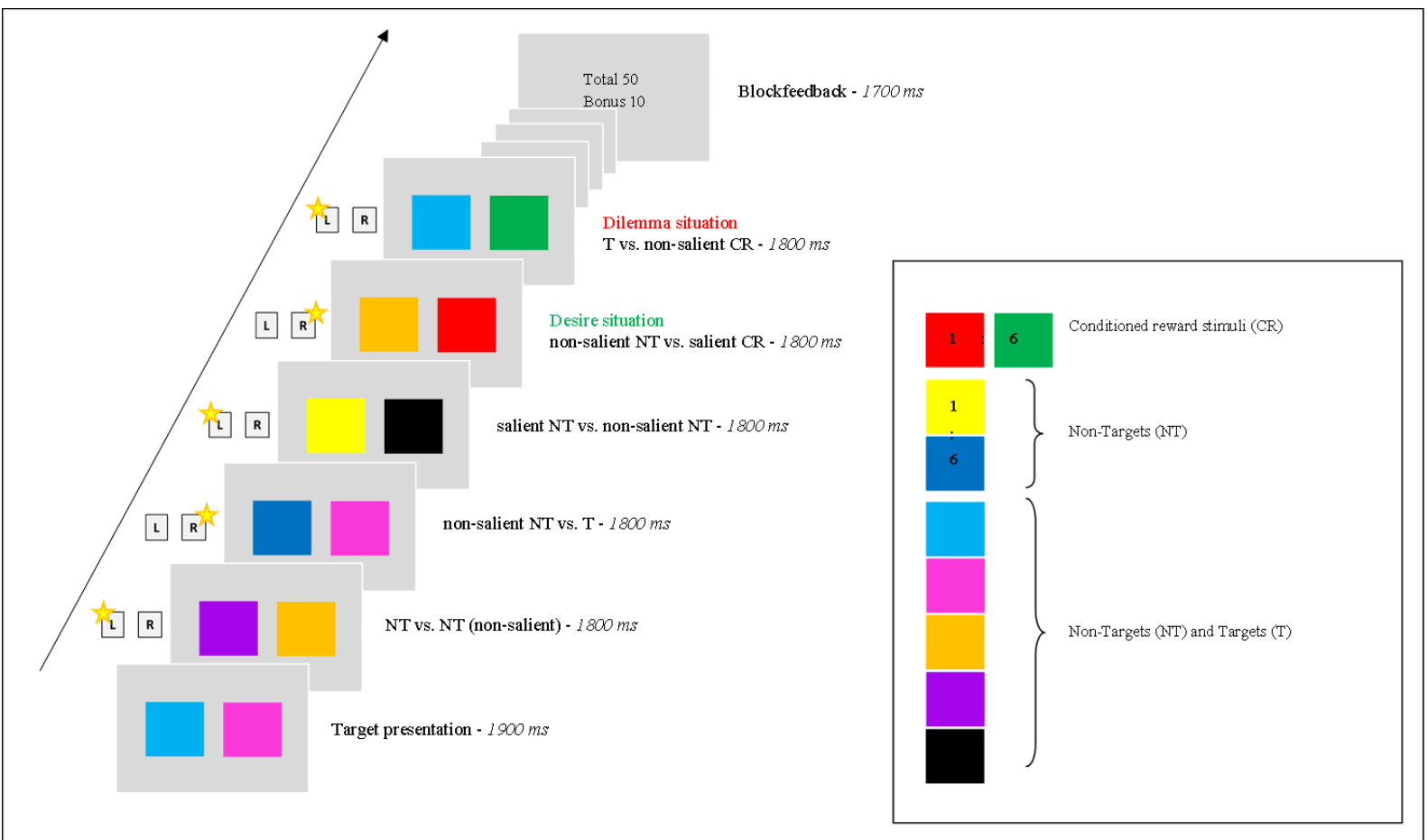

Figure 1. Experimental design of the 'desire-reason dilemma paradigm. The superordinate task goal of each block was to collect all target stimuli and additionally collect all conditioned reward stimuli, when they were presented together with a non-target in the desire situation. However, subjects were forced to reject these stimuli when they were presented together with a target stimulus in the dilemma situation, in order to reach the superordinate task goal of 50 points at the end of each block.

\section{Results}

\section{Behavioral results}

Mean percentages of correct responses and reaction times were compared across salient and nonsalient trials (see Table I for arithmetic mean \pm SEM).

Analysis of performance data revealed significant main effects of salience $\left(F_{(1,25)}=9.486, p=0.005\right)$,

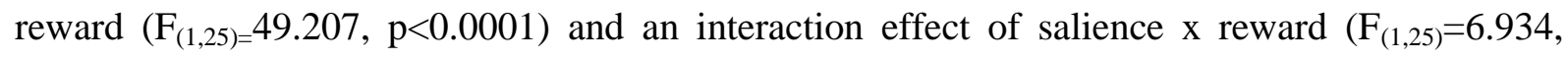
$\mathrm{p}=0.014)$. However, context did not exhibit a significant effect on correct responses $\left(\mathrm{F}_{(1,25)}=2.315\right.$, $\mathrm{p}=0.141$ ). Bonferroni post hoc t-tests revealed significantly higher performance rates for all nonsalient trials compared to salient trials $\left(\mathrm{t}_{(25)}=3.080, \mathrm{p}=0.005\right)$ as well as for all trials without a $\mathrm{CR}$ compared to trials including $\mathrm{CR}\left(\mathrm{t}_{(25)}=7.016, \mathrm{p}<0.0001\right)$.

In addition, reaction time data showed main effects of salience $\left(F_{(1,25)}=30.602, p<0.0001\right)$, reward $\left(\mathrm{F}_{(1,25)}=72.799, \mathrm{p}<0.0001\right)$ and context $\left.\left(\mathrm{F}_{1,25}\right)=213.099, \mathrm{p}<0.0001\right)$, as well as interaction effects of salience $x$ context $\left(\mathrm{F}_{(1,25)}=5.661, \mathrm{p}=0.025\right)$ and reward $\mathrm{x}$ context $\left(\mathrm{F}_{(1,25)}=20.848, \mathrm{p}<0.0001\right)$, 
confirming the successful experimental implementation of saliency in this study. Post hoc t-tests correcting for multiple comparisons uncovered a significant increase of reaction times when subjects responded to the target stimuli presented together with a salient non-target $\left(t_{(25)}=3.836\right.$, $\mathrm{p}=0.001)$ and presented together with a salient $\mathrm{CR}\left(\mathrm{t}_{(25)}=4.245, \mathrm{p}<0.0001\right)$ compared to the corresponding non-salient trials. This may demonstrate enhanced working memory demands in recalling and comparing information regarding the target stimulus and the infrequently presented salient stimuli leading to a prolonged reaction compared to the frequently presented stimuli, replicating previous findings (Gruber et al., 2009; 2010).

\section{FMRI results}

Replicating previous findings (Diekhof and Gruber, 2010), in the desire contrast reliable bottomup activation of the bilateral vStr and VTA (see Table II, (1)) and of an extended bilateral frontoparietal network was found (SI, Table S1). In addition, in the reason contrast simultaneous presentation of $\mathrm{CR}$ and target stimulus led to a significant down-regulation of activation in the bilateral vStr and VTA (see Table III, (4)).

Successful modulation of salience in the present experiment was ensured by implementing both infrequent $\mathrm{CR}$ and infrequent non-targets without a reward association. First, we wanted to examine the effect of infrequent and therewith salient non-targets on the mesolimbic reward system. Indeed, presentation of infrequently presented non-targets compared to the frequently presented non-targets elicited reliable activation of the left vStr, right VTA and further saliencyprocessing brain regions including the left OFC, left IFG and bilateral ACC (Table IV). These brain regions were activated due to saliency per se.

Second, infrequent and therefore salient CR also elicited reward-related activation in the bilateral vStr and VTA (Table II (2); see also SI, Figure S1), as well as in several fronto-parietal brain regions in the desire saliency contrast (SI, Table S2). Further, in the reason saliency contrast we found reduced suppression of reward-related activation in the left vStr and bilateral VTA (Table III, (5)). Interestingly, comparison of the sRC and RC, when it was not allowed to choose the CR (salient or not), revealed an increased activation of the left vStr and bilateral VTA (Figure 2; Table III, (6) left), indicating a boosting of activation in these brain regions due to the saliency of the CR. 


\section{PPI results}

To further examine the effects of the modulation of salience on cortico-subcortical interactions, at first we explored the functional connectivity of the VTA and vStr in the desire saliency and reason saliency contrasts. We observed a significantly increased functional interaction between the right VTA and the right vStr in the sDC compared to the DC (seed: 9 -16-17; Table V (A); see also Figure 3). In addition, we also detected an increased functional coupling between these regions when comparing the sRC with the RC (seed: 9 -16 -17; Table V (B); Figure 3), consistent with the boosting of activation in these brain regions (as shown in Table III). This enhanced functional connectivity was also found between the left vStr and the right vStr as well as between the bilateral VTA in the sDC compared to the DC (seed: -95 -8; Table V (A)). Moreover, both VTA and vStr showed an increased functional coupling with the OFC and the amygdala in the observed contrasts (see Table V (A) + (B)). In a second step, we explored the functional connectivity of further saliency-processing brain regions including the OFC, IFG and ACC, which were additionally activated due to the salient non-target stimuli in the saliency contrast. Once more, we found increased functional interactions between these seed regions and the VTA and/or vStr, in both the SDC > DC and SRC > RC contrasts (see SI, Table S3), demonstrating the existence of multiple increased functional connections between cortical and subcortical brain regions when processing saliency. 
Influence of ventral tegmental area input on cortico-subcortical networks underlying action control and decision making

Table I Percentage of correct responses and reaction times of 26 participants (mean \pm SEM)

\begin{tabular}{|c|c|c|c|}
\hline & & Non-salience & Salience \\
\hline \multirow{2}{*}{ No reward } & $\begin{array}{c}\text { non-target vs. non- } \\
\text { target trials }\end{array}$ & $\begin{array}{c}99.9 \% \pm 0.04 \% \\
749 \mathrm{~ms} \pm 26.3 \mathrm{~ms}\end{array}$ & $\begin{array}{c}99.8 \% \pm 0.2 \% \\
763 \mathrm{~ms} \pm 28.8 \mathrm{~ms}\end{array}$ \\
\hline & $\begin{array}{c}\text { non-target vs. target } \\
\text { trials }\end{array}$ & $\begin{array}{c}98.8 \% \pm 0.2 \% \\
556 \mathrm{~ms} \pm 20.8 \mathrm{~ms}\end{array}$ & $\begin{array}{c}98.1 \% \pm 0.5 \% \\
581 \mathrm{~ms} \pm 19.2 \mathrm{~ms}\end{array}$ \\
\hline \multirow{2}{*}{ Reward } & $\begin{array}{c}C R \text { vs. non-target } \\
\text { trials }\end{array}$ & $\begin{array}{c}93.7 \% \pm 1.0 \% \\
849 \mathrm{~ms} \pm 27.3 \mathrm{~ms}\end{array}$ & $\begin{array}{c}91.2 \% \pm 1.3 \% \\
858 \mathrm{~ms} \pm 27.4 \mathrm{~ms}\end{array}$ \\
\hline & $C R$ vs. target trials & $\begin{array}{c}93.2 \% \pm 0.9 \% \\
605 \mathrm{~ms} \pm 19.0 \mathrm{~ms}\end{array}$ & $\begin{array}{c}91.9 \% \pm 1.3 \% \\
640 \mathrm{~ms} \pm 23.0 \mathrm{~ms}\end{array}$ \\
\hline Overall & & $\begin{array}{c}96.4 \% \pm 0.5 \% \\
690 \mathrm{~ms} \pm 16.4 \mathrm{~ms}\end{array}$ & $\begin{array}{c}95.3 \% \pm 0.6 \% \\
710 \mathrm{~ms} \pm 16.3 \mathrm{~ms}\end{array}$ \\
\hline
\end{tabular}

Table II Reward-related brain activations in the desire contrast (DC), desire saliency contrast (sDC) and the comparison between them

\begin{tabular}{|c|c|c|c|c|}
\hline Region & Desire contrast & $\begin{array}{l}\text { Desire saliency } \\
\text { contrast } \\
\text { (2) }\end{array}$ & $\begin{array}{l}\text { Desire saliency } \\
\text { contrast > } \\
\text { Desire contrast }\end{array}$ & $\begin{array}{l}\text { Desire contrast } \\
\text { Desire saliency } \\
\text { contrast }\end{array}$ \\
\hline & \multicolumn{4}{|c|}{ MNI coordinates ( $t$-value) } \\
\hline $\begin{array}{l}\mathbf{R} \text { dorsal/ventral } \\
\text { Striatum }\end{array}$ & $128-2(4.78)^{*}$ & $1281(4.73)^{* *}$ & n.s. & n.s. \\
\hline $\begin{array}{l}\text { L dorsal/ventral } \\
\text { Striatum }\end{array}$ & $-158-2(4.16)^{*}$ & $-128-2(3.69)^{*}$ & n.s. & $-65-11(2.21)^{+}$ \\
\hline $\begin{array}{l}\text { R } \\
\text { midbrain/VTA }\end{array}$ & $9-19-20(4.53)^{*}$ & $9-19-14(2.81)^{*}$ & n.s. & n.s. \\
\hline $\begin{array}{l}\text { L } \\
\text { midbrain/VTA }\end{array}$ & $-6-22-17(3.82)^{*}$ & $-3-22-26(2.65)$ & n.s. & n.s. \\
\hline
\end{tabular}


Abbreviations: L, left; n.s., not significant; R, right.

Activations are reported at $\mathrm{p}<0.005$, uncorrected; ${ }^{+} \mathrm{p}<0.05$, uncorrected, with a minimum cluster size of 10 voxels; *p<0.05 FWE-corrected for small volume; **p<0.05, FWE-corrected (whole brain).

Table III Reward-related brain activations in the reason contrast (RC), reason saliency contrast (sRC) and the comparison between them

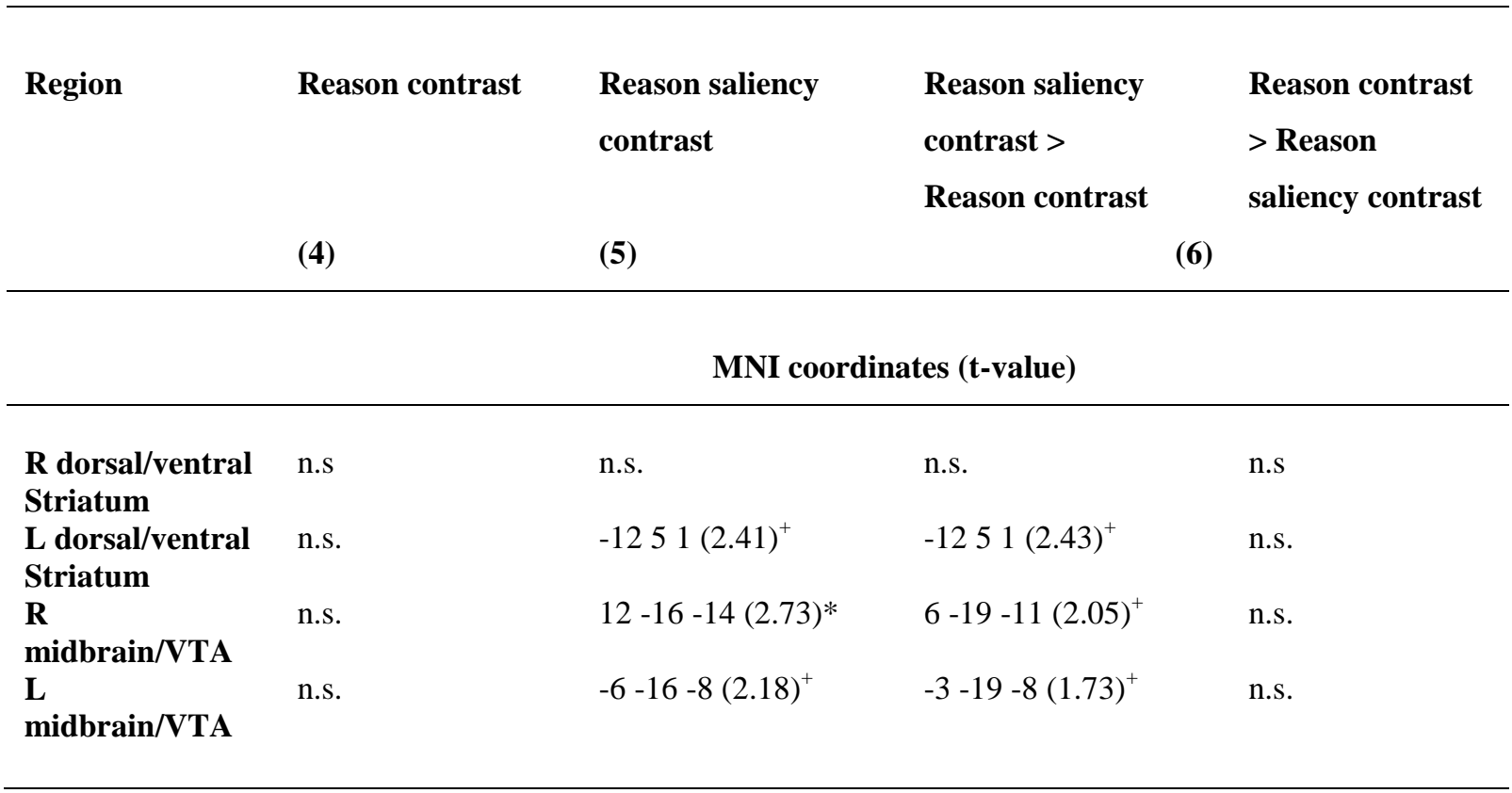

Abbreviations: L, left; n.s., not significant; R, right.

Activations are reported at ${ }^{+} \mathrm{p}<0.05$, uncorrected, with a minimum cluster size of 10 voxels; $* \mathrm{p}<0.05$ FWE-corrected for small volume; $* * \mathrm{p}<0.05$, FWE-corrected (whole brain). 
Influence of ventral tegmental area input on cortico-subcortical networks underlying action control and decision making

Table IV Saliency-related brain activations in the saliency contrast (SC) without a reward association

Region Saliency contrast

MNI coordinates ( $t$-value)

L dorsal/ventral Striatum

R midbrain/VTA

L OFC

L IFG/ pars triangularis

R BA 6/ precentral gyrus

L BA 6/ precentral gyrus

$\mathrm{L}$ fronto-opercular cortex/ anterior insular cortex

$R$ dorsal ACC

$\mathrm{L}$ dorsal ACC

$\mathrm{L}$ intraparietal cortex

$\mathrm{L}$ inferior parietal lobule

$\mathbf{R}$ middle temporal gyrus

$\mathbf{R}$ extrastriate occipital cortex

L extrastriate occipital cortex

L parahippocampal gyrus

L putamen

L/R superior colliculus

$\mathbf{R}$ medial globus pallidus

$$
\begin{aligned}
& -95-8(2.31)^{+} \\
& 9-16-17(2.50)^{*} \\
& -5429-5(1.74)^{+} \\
& -54291(1.81)^{+} \\
& 42-431(2.09)^{+} \\
& -42-731(2.20)^{+} \\
& -21171(2.08)^{+}
\end{aligned}
$$

$$
\begin{aligned}
& 12828(2.52)^{+} \\
& -181125(1.85)^{+} \\
& -21-4640(2.11)^{+} \\
& -51-2540(2.49) \\
& 545-20(2.11)^{+} \\
& 24-10016(2.84) \\
& -15-94-8(2.61) \\
& \begin{array}{lll}
-21 & -52 & -8 \\
(2.28)^{+}
\end{array} \\
& -245-5(2.13)^{+} \\
& 0-311(2.22)^{+}
\end{aligned}
$$

Abbreviations: ACC, anterior cingulate cortex, BA, brodmann area; L, left; n.s., not significant; OFC, orbitofrontal cortex; R, right.

Activations are reported at $\mathrm{p}<0.005$, uncorrected, with a minimum cluster size of 10 voxels; ${ }^{+} \mathrm{p}<0.05$, uncorrected, with a minimum cluster size of 10 voxels; ${ }^{*} \mathrm{p}<0.05$ FWE-corrected for small volume. 
Table V Increased psychophysiological interactions of the right VTA and left vStr in the desire saliency contrast compared to the desire contrast (A) and the reason saliency contrast compared to the reason contrast $(B)$

\begin{tabular}{|c|c|c|}
\hline \multirow[t]{2}{*}{ Region } & Seed area & Seed area \\
\hline & R VTA (9 -16 -17) & L vStr (-9 5 -8 \\
\hline
\end{tabular}

(A)

$\mathbf{S D C}>\mathbf{D C}$
(B)

$\mathbf{S R C}>\mathbf{R C}$
(A)

SDC $>$ DC
(B)

sRC > RC

MNI coordinates (t-value)

$\begin{array}{lllll}\begin{array}{l}\text { R dorsal/ventral } \\ \text { Striatum }\end{array} & 917-8(1.82)^{+} & 926-11(2.45)^{+} & 158-8(2.95)^{*} & \text { n.s. } \\ \begin{array}{l}\text { L dorsal/ventral } \\ \text { Striatum } \\ \text { R midbrain/ }\end{array} & \text { n.s. } & \text { n.s. } & \text { n.s. } & \text { n.s. } \\ \begin{array}{l}\text { VTA } \\ \text { L midbrain/ }\end{array} & \text { n.s. } & 6-10-17(2.20)^{+} & 6-10-14(2.66)^{+} & \text {n.s. } \\ \begin{array}{l}\text { VTA } \\ \text { R anterior/ }\end{array} & \text { n.s. } & -6-10-17(1.86)^{+} & -9-19-8(1.83)^{+} & \text {n.s. } \\ \begin{array}{l}\text { central OFC } \\ \text { L anterior/ } \\ \text { central OFC }\end{array} & \text { n.s. } & 3953-11(2.24) & 2738-8(2.37)^{+} & 1532-11(2.51)^{+} \\ \mathbf{L} \text { amygdala } & \text { n.s. } & -2153-5(2.18)^{+} & -2138-14(2.32)^{+} & -1835-14(3.30)^{*} \\ & & \text { n.s. } & -21-4-14(3.30)^{*} & -30-1-8(3.06)\end{array}$

Abbreviations: L, left; n.s., not significant; OFC, orbitofrontal cortex; R, right.

Activations are reported at $\mathrm{p}<0.005$, uncorrected; ${ }^{+} \mathrm{p}<0.05$, uncorrected; ${ }^{*} \mathrm{p}<0.05$ FWE-corrected for small volume. 


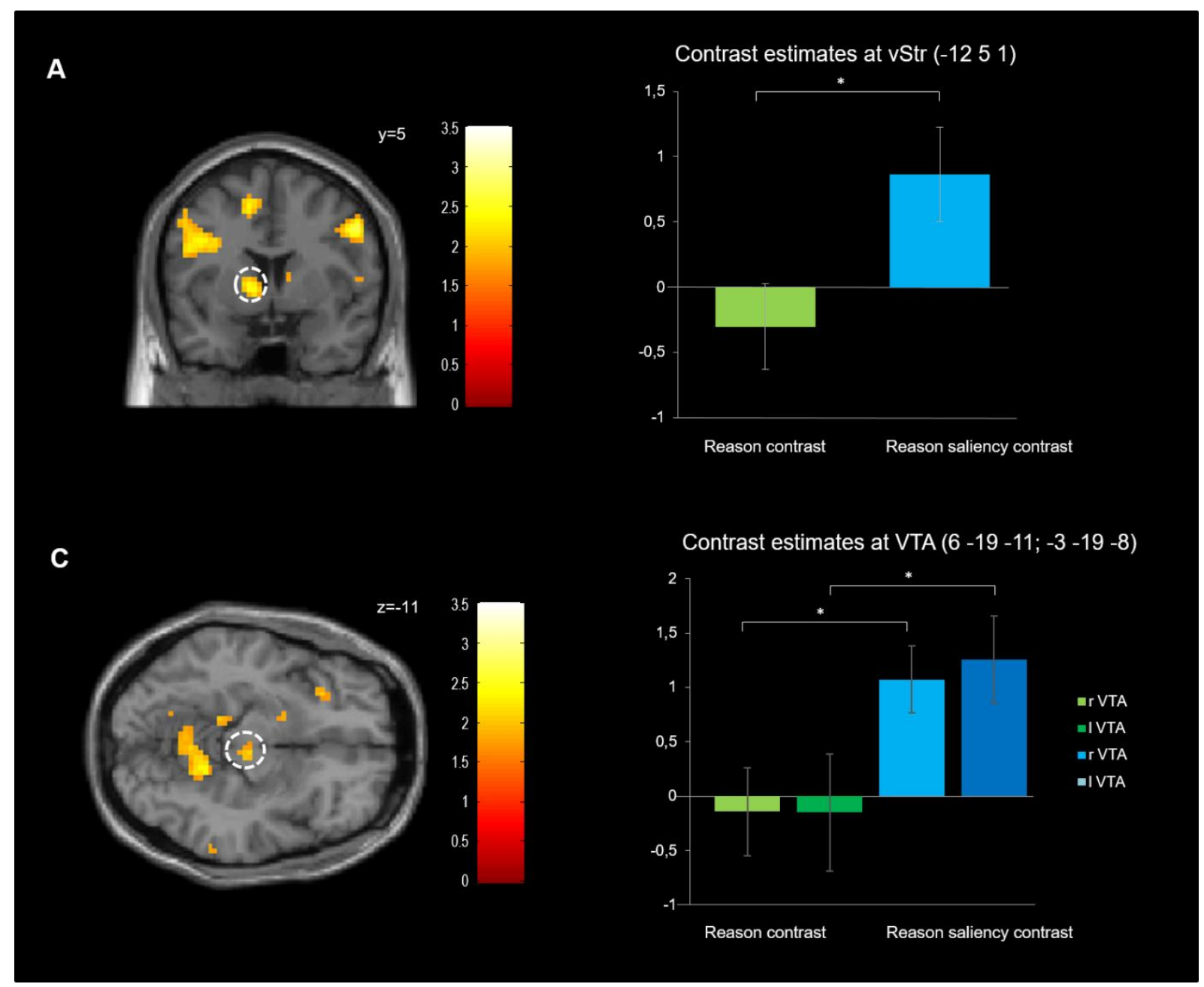

Figure 2. Reward-related brain activations in the comparison of the reason saliency contrast (sRC) vs. reason contrast (RC). A left Increased activation of the left vStr; right contrast estimates at the vStr (mean $\pm \mathrm{SEM} ;{ }^{*} \mathrm{p}<0.05$ ). B left Increased activation of the bilateral VTA; right contrast estimates at the VTA (mean $\pm \mathrm{SEM}$; ${ }^{*} \mathrm{p}<0.05$ ). Activation was thresholded at $\mathrm{p}<0.05$, uncorrected. T-values are indicated by color bars. Regions listed in Table III. For more details see Supplementary Table S2. 


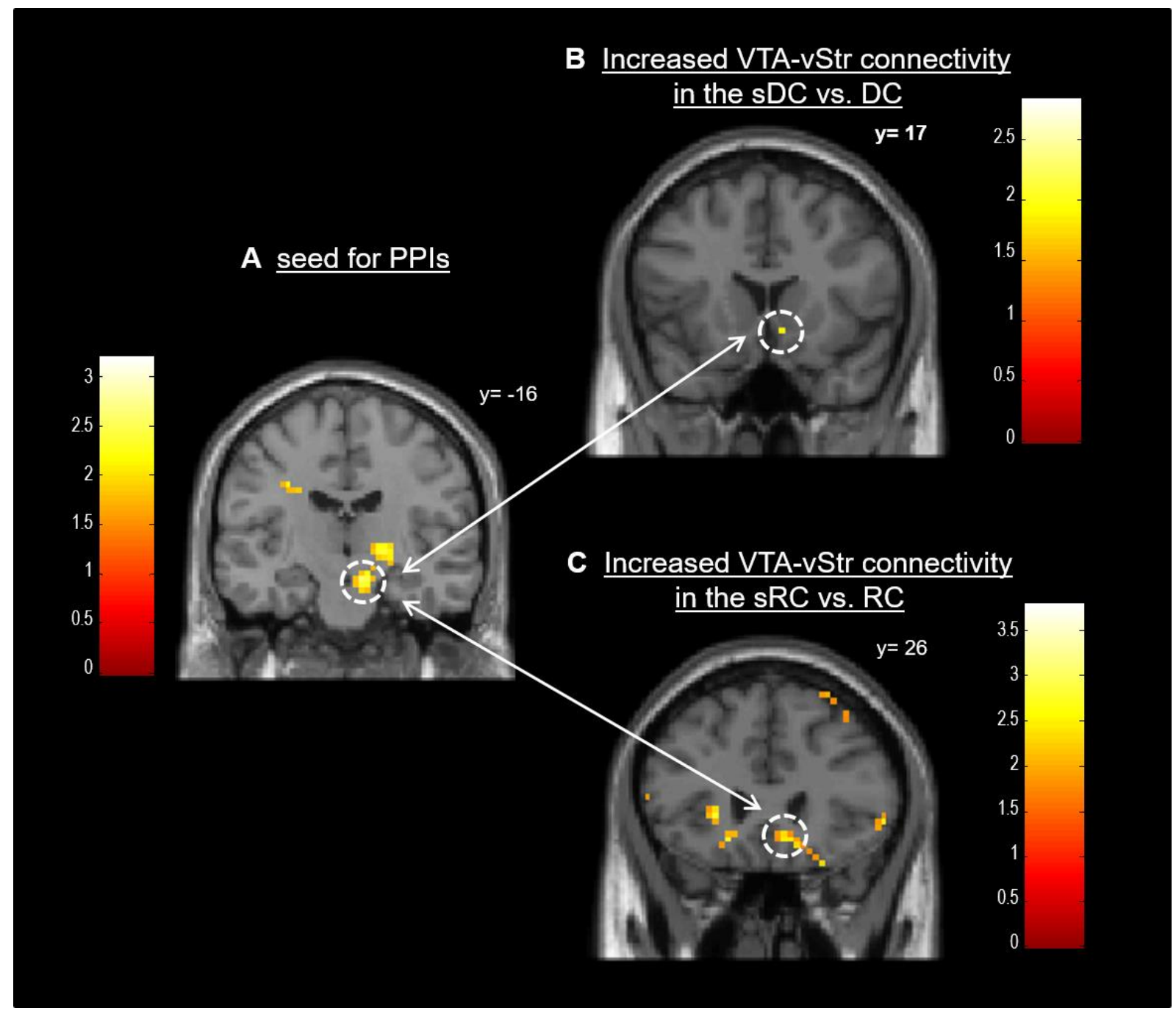

Figure 3. Increased VTA-vStr connectivity. Increased functional interaction between (A) right VTA and right vStr in the direct comparison of the (B) desire saliency contrast vs. desire contrast and $(\mathbf{C})$ reason saliency contrast vs. reason contrast. Activation was thresholded at $\mathrm{p}<0.05$, uncorrected. T-values are indicated by color bars. Regions listed in Table V. 


\section{Discussion}

In the present study we investigated the impact of the modulation of salience on the functional activity and connectivity of the human mesolimbic reward system, focusing on the functional response of the VTA and vStr to infrequent events in a reward-based decision making task.

An important new feature of the applied DRD paradigm is the introduced factor saliency as implemented by the manipulation of both rewarding und neutral stimuli through relative frequency. Infrequent stimuli with long intervals between consecutive events are unexpected and hence more salient. Behavioral results confirmed the successful experimental implementation of saliency in this study, as analyses of reaction time data revealed significant main effects of salience, reward and context. Reaction times for trials including target stimuli plus infrequent reward stimuli were significantly longer than for trials including target stimuli plus frequent reward stimuli. The same applies to presented trials encompassing target stimuli plus infrequent non-target stimuli compared to trials with frequent non-target stimuli. Infrequent stimuli independent of the reward association increased the reaction times to target stimuli, demonstrating the occurrence of contextual mismatch effects that may impose increased demands on cognitive control processes (Gruber et al., 2009).

In line with these behavioral data, neuroimaging findings confirmed the successful experimental implementation of saliency by showing that infrequently presented neutral stimuli led to an increased activation of the VTA and vStr due to saliency per se. Additionally, these salient stimuli elicited reliable activation of the left OFC, left IFG and bilateral ACC. Previous studies have used oddball paradigms to assess brain responses to infrequent salient stimuli without a reward association, by reporting on the one hand no striatal activity (Clark et al., 2000; Kirino et al., 2000) and on the other hand VTA activity in response to infrequent behaviorally relevant deviants in a cued task-switching paradigm (Gruber et al., 2010). We could extend these findings by showing that the VTA as well as the vStr were significantly activated in response to these infrequent stimuli. Moreover, the results of the present study reveal that activity in the VTA and vStr increased in response to both frequent and infrequent rewarding stimuli, replicating previous findings (Diekhof and Gruber, 2010; Diekhof et al., 2012a, b). There is growing consensus that the brain computes and compares value and saliency signals at the time of decision making (Rangel et al., 2008). Value signals provide a measure of the desirability of a stimulus, constituted by the associated amount of reward. Saliency signals, in turn, provide a measure of the importance of the stimulus, relating to motivational and attentional processes in the brain (Rangel et al., 2008). On the biological level, there is evidence for independent dopaminergic processing pathways of reward and saliency leading to the assumption that midbrain dopamine neurons are 
Influence of ventral tegmental area input on cortico-subcortical networks underlying action control and decision making

not homogeneous (Matsumoto and Hikosaka, 2009; Matsumoto and Takada, 2013). It is hypothesized that on the one side, a proportion of neurons respond to rewarding and rewardpredicting stimuli, encoding the motivational value for positive outcomes, engendering value learning and seeking behavior (Berridge, 2012). Alternatively, other neuron populations in the midbrain encode a motivational salience signal by responding to generally salient stimuli, triggering orienting and explorative behavior (Bromberg-Martin et al., 2010).

Comparison of infrequent reward trials and frequent reward trials did not show stronger mesolimbic activation in response to the salient features of the reward stimuli (see SI, Figure S1). One previous study dissociated value and saliency signals at the time of choice and showed that the vStr was modulated by both value and salience (Litt et al., 2011). Furthermore, it was found that the vStr also correlates with both saliency and valence during the anticipation of probabilistic rewards (Cooper and Knutson, 2008). However, the implementation of saliency between these studies and the present study varied widely. In the applied DRD paradigm, infrequent rewards combined both rewarding and salient attributes. It is reasonable that the underlying neural activities may interfere in our study and that a higher spatial resolution of fMRI is needed to disentangle the overlapping activities.

Contrary to previous findings (Diekhof and Gruber, 2010; Diekhof et al. 2012a, b), the mesolimbic reward system showed significantly increased activation in response to the salient reward stimuli when presented together with target stimuli (sRC) as compared to the frequent and therefore less salient reward stimuli (RC), indicating a boosting of activation in the vStr and VTA (see Figure 2). PPI analyses could confirm this assumption by revealing a significantly increased functional connectivity between the VTA and vStr in both the comparison of the desire saliency contrast with the desire contrast and the reason saliency contrast compared to the reason contrast (see Figure 3). A previous study has provided evidence for inhibitory influences of the anteroventral prefrontal cortex (avPFC) on the mesolimbic dopamine system during selfcontrolled decisions (Diekhof and Gruber, 2010). However, the strong functional coupling between VTA and vStr led to the assumption that the saliency-modulated dopamine input from the VTA to the vStr may be stronger compared to inhibitory influences of the avPFC and in turn, that the saliency signal in the VTA apparently was not suppressed by prefrontal regulatory mechanisms (Bromberg-Martin et al., 2010; Macpherson et al., 2014), leading to the boosting of activation in situations where top-down control was needed. In addition to that, the VTA and vStr showed an increased functional coupling with the OFC and the amygdala. Moreover, we could show multiple increased functional interactions between OFC, IFG, ACC and subcortical brain regions. As part of the reward circuit, the OFC has been shown to play a central role in processing of incentive and motivational value in animals (Schultz et al., 2000; Sesack and Grace, 2010), in 
Influence of ventral tegmental area input on cortico-subcortical networks underlying action control and decision making

detecting motivational significant events outside the current focus of attention, and it has been further shown to exhibit an increased functional interaction with the VTA when processing salient events in humans (Diekhof et al., 2009). It was previously hypothesized that dopamine may not signal the motivationally significance of stimuli itself but rather regulate orbitofrontal and amygdalar glutamatergic inputs to striatal regions, which is necessary for adaptive decision making (Horvitz, 2000). Animal studies provided evidence that stimulation of the vStr influenced OFC activity and possibly connectivity (Ewing and Grace, 2013) and in turn, lesions in OFC led to changes in striatal dopamine levels (Clarke et al., 2014), demonstrating the necessity of the interaction between OFC and mesolimbic structures in guiding adaptive behavior. In addition, the dorsal ACC together with the insula constitute the salience network, which is mainly involved in sensory perception and the coordination of behavioral responses (Lamichhane et al., 2016). Furthermore, the ACC has also commonly observed in oddball processing and target detection (Downar et al., 2001; Brázdil et al., 2005) and also in reversal learning studies (Kringelbach and Rolls, 2003). Likewise, activations of the IFG has been found to be evoked by both response conflicts and by contextual mismatches (Gruber et al., 2009) as well as in response inhibition and instrumental learning in go/no-go tasks (Guitart-Masip et al., 2012). Overall, this study provided clear evidence for the importance of increased functional interactions between cortical saliencyprocessing brain regions and mesolimbic structures of the reward system in adaptive decision making.

In conclusion, these findings contribute to the growing understanding of how brain mechanisms may process and integrate the influence of salient and rewarding information on decision making. We could show that coding of infrequent and therefore salient events led to a significant boosting of activation in the VTA and vStr. Specifically, we further revealed significantly increased functional coupling between these key regions of the reward system underlying the boosting of activation. Moreover, our findings highlight the existence of multiple increased functional interactions between brain regions within and beyond the mesolimbic reward system underlying adaptive processing of salient events and successful behavioral decision making. 
Influence of ventral tegmental area input on cortico-subcortical networks underlying action control and decision making

\section{Acknowledgments}

We would like to thank Ilona Pfahlert and Britta Perl as well as the staff of the unit 'MR-Research in Neurology and Psychiatry' at the University Medical Center Göttingen (Germany) for help with data acquisition.

This work was partially funded by a Deutsche Forschungsgemeinschaft (DFG) grant to Oliver Gruber (GR1950/8-1). 


\subsection{Appendix: Supplementary data}

\section{Material and Methods}

\section{Experimental Task}

In order to avoid a systematic bias by temporally correlated trials possibly leading to confounding effects we matched all trials (two colors presented simultaneously) regarding their preceding and following trials. Furthermore, salient trials (including either a salient non-target or a salient CR) were positioned at least 4 trials apart from each other, allowing for a modulation of the blood oxygenation level-dependent (BOLD) response for all salient events. In total, subjects completed 126 blocks over the course of three functional runs. The two relevant target colors for the upcoming task block were shown for $1900 \mathrm{~ms}$ (preceded by a $100 \mathrm{~ms}$ blank screen delay and followed by a $200 \mathrm{~ms}$ blank screen). The relevant target colors changed every task block. Afterwards, individual trials with two colored squares where presented for $1800 \mathrm{~ms}$. At the end of each task block, a feedback indicated the overall outcome together with the collected bonus points. Feedback was presented for $1700 \mathrm{~ms}$, preceded by a $200 \mathrm{~ms}$ blank screen and followed by a $100 \mathrm{~ms}$ blank screen before the next task block began. In addition, at the end of one functional run a total feedback was presented for $1600 \mathrm{~ms}$, which indicated the overall outcome of the run. Failure to implement the superordinate task goal or failure to answer within $1800 \mathrm{~ms}$ led to zero outcome at the end of the current task block. Overall points acquired over the course of the three runs were cashed into real money. Subjects could receive up to $30 €$ based on their task performance which were added to the general allowance of $20 €$ for participation.

\section{FMRI data acquisition and analysis}

At the individual subject level, statistical analyses were carried out using a general linear model, comprising 10 independent regressors (four individual trial types: trials comprising a non-target paired with a non-target (non-target vs. non-target), non-target paired with a target (non-target vs. target), CR paired with a non-target (CR vs. non-target) and CR paired with a target (CR vs. target) each both for the non-salient trials and for the salient trials, the block cues indicating the target stimuli and the block feedback. Only correctly answered trials were included in the analysis of the event-related fMRI study. A vector representing the temporal onset of stimulus presentation was convolved with a canonical hemodynamic response function (hrf) to produce a predicted 
Influence of ventral tegmental area input on cortico-subcortical networks underlying action control and decision making

hemodynamic response to each experimental condition. Linear t-contrasts were defined for assessing the specific effects of each condition of interest. Group effects were assessed by a second level full factorial model (factors saliency and experimental trial type) based on single subject contrast images.

As the analysis of the imaging data pursued the goal of investigating reward circuit activation that occurred both in association with salient and non-salient events with a reward association and with salient events without a reward association, we compared brain activation from the following subtraction contrasts, namely (1) the contrast of CR vs. non-target trials against non-target vs. non-target trials (desire contrast (DC)), (2) the comparison of salient CR vs. non-target trials against salient non-target vs. non-target trials (desire saliency contrast (sDC)), (3) the comparison of DC against sDC and vice versa, (4) the comparison of CR vs. target trials against non-target vs. target trials (reason contrast (RC)), (5) the comparison of salient CR vs. target trials against salient non-target vs. target trials (reason saliency contrast (sRC)), (6) the comparison of RC against sRC and vice versa as well as (7) the comparison of salient non-target vs. non-target trials against nontarget vs. non-target trials, allowing to separately investigate effects that were attributable to infrequency per se without a reward association (saliency contrast (SC)).

\section{Psychophysiological interaction (PPI) analysis}

Using Matlab and SPM8, the hemodynamic signals were first deconvolved using a parametric empirical Bayesian formulation and mean-corrected. Subsequently, the PPI term was built separately by multiplying the deconvolved and mean-corrected BOLD signal with the psychological vector. After convolution with the hrf, mean correction, and orthogonalization, the three regressors (PPI term, physiological vector, and psychological vector) went into the statistical analysis to determine context-dependent changes of functional connectivity over and above any main effect of task or any main effect of activity in the corresponding brain areas. In the PPI contrasts, the PPI term was computed against implicit baseline. Random-effect analyses were performed on single-subject PPI contrast images with a statistical search criterion of $\mathrm{p}<0.05$, uncorrected. Further, small volume correction was performed for the bilateral orbitofrontal cortex (OFC $\pm 1839-18 ; 6 \mathrm{~mm}$ sphere, taken from Diekhof et al., 2011) and for the bilateral amygdala ( $\pm 24-6-18 ; 6$ mm sphere, taken from Krämer and Gruber, 2015). 
Table S1 Reward-related brain activations in the desire contrast (DC), desire saliency contrast ( $\mathrm{sDC}$ ) and the comparison between them

\begin{tabular}{|c|c|c|c|c|}
\hline Region & Desire contrast & $\begin{array}{l}\text { Desire saliency } \\
\text { contrast }\end{array}$ & $\begin{array}{l}\text { Desire saliency } \\
\text { contrast > } \\
\text { Desire contrast }\end{array}$ & $\begin{array}{l}\text { Desire contrast > } \\
\text { Desire saliency } \\
\text { contrast }\end{array}$ \\
\hline
\end{tabular}

MNI coordinates ( $t-v a l u e)$

\begin{tabular}{|c|c|c|c|c|}
\hline $\begin{array}{l}\mathbf{R} \text { dorsal/ventral } \\
\text { Striatum }\end{array}$ & $128-2(4.78)^{*}$ & $1281(4.73)^{* *}$ & n.s. & n.s. \\
\hline $\begin{array}{l}\text { L dorsal/ventral } \\
\text { Striatum }\end{array}$ & $-158-2(4.16)^{*}$ & $-128-2(3.69)^{*}$ & n.s. & $-65-11(2.21)^{+}$ \\
\hline $\begin{array}{l}\text { R } \\
\text { midbrain/VTA }\end{array}$ & $9-19-20(4.53)^{*}$ & $9-19-14(2.81)^{*}$ & n.s. & n.s. \\
\hline $\begin{array}{l}\text { L } \\
\text { midbrain/VTA }\end{array}$ & $-6-28-14(3.82)$ & $-3-22-26(2.65)$ & n.s. & n.s. \\
\hline$R$ avPFC & $2456-8(2.24)^{+}$ & $246510(2.37)^{+}$ & $36411(2.31)^{+}$ & $1841-2(2.64)$ \\
\hline$L$ avPFC & $-2753-5(2.24)^{+}$ & $-30474(3.11)$ & n.s. & n.s. \\
\hline $\mathrm{L}$ anterior OFC & n.s. & $-3653-8(2.11)^{+}$ & $-3944-11(1.79)^{+}$ & n.s \\
\hline $\mathrm{L}$ central OFC & n.s. & $-2132-14(2.19)^{+}$ & n.s. & n.s. \\
\hline R medial OFC & $2141-11(2.42)^{+}$ & $2141-14(3.32)$ & n.s. & n.s. \\
\hline R MFG & $422625(4.03)$ & $482625(5.09)^{* *}$ & n.s. & n.s. \\
\hline L MFG & n.s. & $-452922(4.62)^{* *}$ & n.s. & n.s. \\
\hline $\begin{array}{l}\text { L IFG/ pars } \\
\text { triangularis }\end{array}$ & $-42825(5.84)^{* *}$ & $-421728(4.65)^{* *}$ & n.s. & $-541119(2.24)^{+}$ \\
\hline $\begin{array}{l}\text { R MFG } \\
\text { posterior third/ } \\
\text { premotor cortex }\end{array}$ & 33267 (3.37) & n.s. & n.s. & n.s. \\
\hline $\begin{array}{l}\text { L MFG } \\
\text { posterior third/ } \\
\text { premotor cortex }\end{array}$ & $-27-761(3.40)$ & $-30-752(2.09)^{+}$ & n.s. & n.s. \\
\hline $\begin{array}{l}\text { L primary } \\
\text { motor cortex }\end{array}$ & $-36-1958(3.62)$ & n.s. & n.s. & n.s. \\
\hline $\begin{array}{l}\text { R BA 6/ } \\
\text { precentral gyrus }\end{array}$ & $33-437(2.22)^{+}$ & $24-458(3.00)$ & n.s. & n.s. \\
\hline $\begin{array}{l}\text { L BA } 6 \\
\text { /precentral } \\
\text { gyrus }\end{array}$ & $-36-446(4.78)^{* *}$ & $-30-752(2.09)^{+}$ & n.s. & n.s. \\
\hline $\begin{array}{l}\text { R postcentral } \\
\text { gyrus }\end{array}$ & $45-1028(2.49)^{+}$ & n.s. & n.s. & $42-1028(3.18)$ \\
\hline R IFJ & $42531(3.55)$ & $481152(2.46)^{+}$ & $30840(2.07)^{+}$ & n.s. \\
\hline L IFJ & n.s. & $-45534(4.62)^{* *}$ & $-361443(1.99)^{+}$ & n.s. \\
\hline $\begin{array}{l}\mathbf{R} \text { fronto- } \\
\text { opercular } \\
\text { cortex/ anterior } \\
\text { insular cortex }\end{array}$ & $3026-5(3.22)$ & $3323-5(5.77)^{* *}$ & $3617-11(2.40)^{+}$ & n.s. \\
\hline L fronto- & $-27231(3.45)$ & n.s. & n.s. & $-21204(2.40)^{+}$ \\
\hline
\end{tabular}




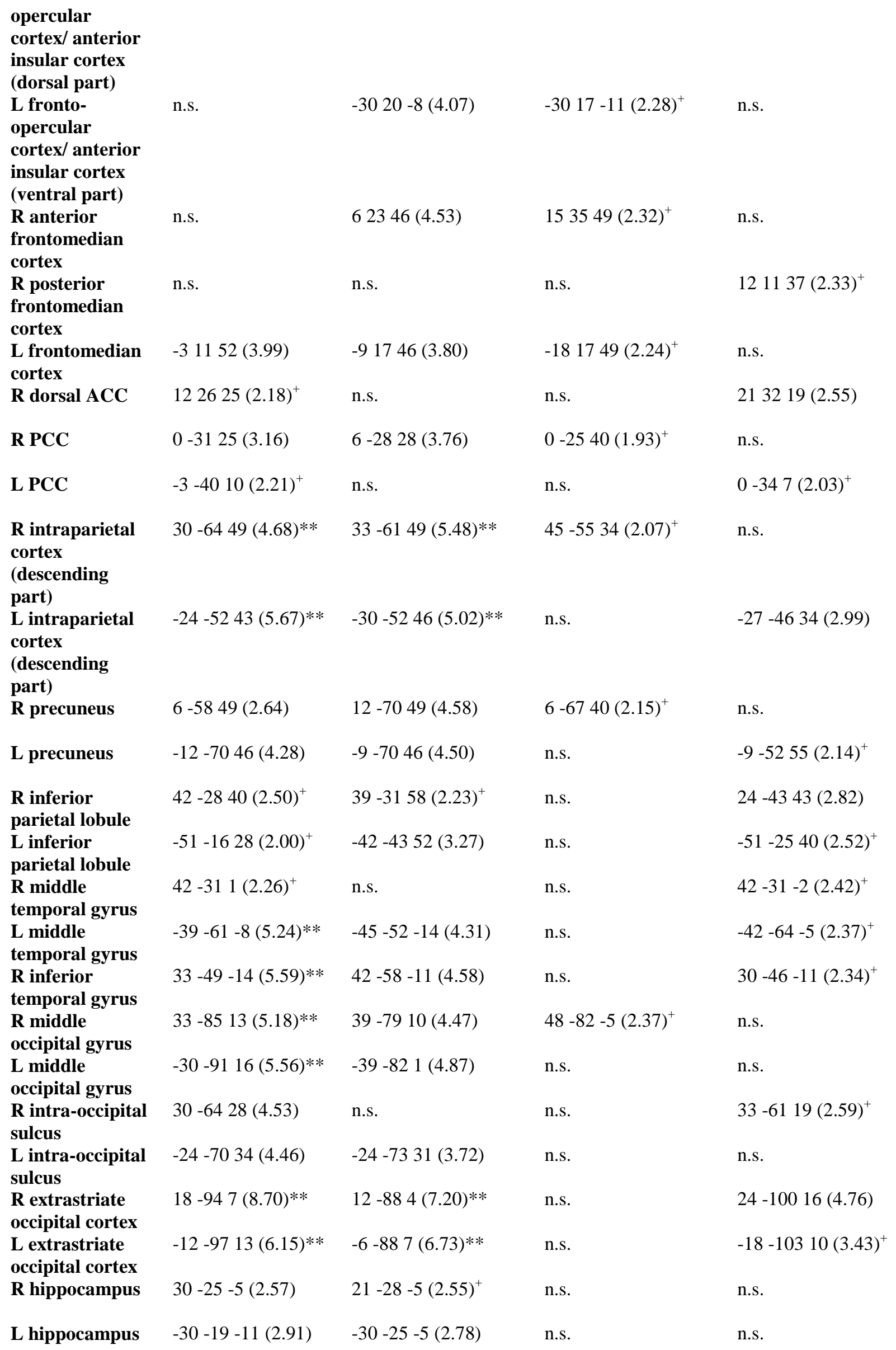


Influence of ventral tegmental area input on cortico-subcortical networks underlying action control and decision making

\begin{tabular}{lllll}
\hline L caudate & $-15-110(5.29)^{* *}$ & $-1287(4.56)$ & n.s. & $-211416(2.47)$ \\
$\mathbf{R}$ thalamus & $12-1013(5.72)^{* *}$ & $12-1610(4.24)$ & n.s. & n.s. \\
L thalamus & $-12-1610(4.07)$ & $-9-131(4.57)$ & n.s. & n.s. \\
\hline
\end{tabular}

Abbreviations: avPFC, anteroventral prefrontal cortex; BA, brodmann area; IFJ, inferior frontal junction; L, left; MFG, middle frontal gyrus; MCC, middle cingulate cortex; n.s., not significant; OFC, orbitofrontal cortex; PCC, posterior cingulate cortex; R, right; SFG, superior frontal gyrus. Activations are reported at $\mathrm{p}<0.005$, uncorrected, with a minimum cluster size of 10 voxels, if not otherwise indicated; ${ }^{+} \mathrm{p}<0.05$, uncorrected, with a minimum cluster size of 10 voxels; ${ }^{*} \mathrm{p}<0.05$ FWE-corrected for small volume (for SVC we used spheres with a radius of 6 and $8 \mathrm{~mm}$, around the maxima reported by Diekhof and Gruber, 2010 and Diekhof et al., 2012a,b); **p<0.05, FWEcorrected (whole brain).

Table S2 Reward-related brain activations in the reason contrast (RC), reason saliency contrast (sRC) and the comparison between them

\begin{tabular}{|c|c|c|c|c|}
\hline Region & Reason contrast & $\begin{array}{l}\text { Reason saliency } \\
\text { contrast }\end{array}$ & $\begin{array}{l}\text { Reason saliency } \\
\text { contrast }> \\
\text { Reason contrast }\end{array}$ & $\begin{array}{l}\text { Reason contrast > } \\
\text { Reason saliency } \\
\text { contrast }\end{array}$ \\
\hline
\end{tabular}

MNI coordinates ( $t-v a l u e)$

\begin{tabular}{|c|c|c|c|c|}
\hline $\begin{array}{l}\mathbf{R} \text { dorsal/ventral } \\
\text { Striatum }\end{array}$ & n.s & n.s. & n.s. & n.s \\
\hline $\begin{array}{l}\text { L dorsal/ventral } \\
\text { Striatum }\end{array}$ & n.s. & $-1251(2.41)^{+}$ & $-1251(2.43)^{+}$ & n.s. \\
\hline $\begin{array}{l}\mathrm{R} \\
\text { midbrain/VTA }\end{array}$ & n.s. & $12-16-14(2.73)^{*}$ & $6-19-11(2.05)^{+}$ & n.s. \\
\hline $\begin{array}{l}\text { L } \\
\text { midbrain/VTA }\end{array}$ & n.s. & $-6-16-8(2.18)^{+}$ & $-3-19-8(1.73)^{+}$ & n.s. \\
\hline$R$ avPFC & n.s. & $12654(2.08)^{+}$ & n.s. & n.s. \\
\hline R MFG & n.s. & $392322(3.68)$ & $452316(2.73)$ & n.s. \\
\hline L MFG & n.s. & -452028 (3.28) & n.s. & n.s. \\
\hline $\begin{array}{l}\text { R MFG/ } \\
\text { premotor cortex }\end{array}$ & $36558(2.61)$ & n.s. & n.s. & n.s. \\
\hline $\begin{array}{l}\text { L MFG/ } \\
\text { premotor cortex }\end{array}$ & $-30-158(2.18)^{+}$ & $-39-437(3.21)$ & $-57-743(1.98)^{+}$ & n.s. \\
\hline $\begin{array}{l}\text { L postcentral } \\
\text { gyrus }\end{array}$ & $-18-3461(2.82)$ & n.s. & n.s. & n.s. \\
\hline R IFJ & n.s. & 51834 (3.14) & $45-437(2.74)$ & n.s. \\
\hline L IFJ & n.s. & $-42231(3.33)$ & $-45828(2.49)^{+}$ & n.s. \\
\hline $\begin{array}{l}\text { L fronto- } \\
\text { opercular } \\
\text { cortex/ anterior } \\
\text { insular cortex }\end{array}$ & n.s. & $-27261(3.51)$ & $-30261(3.50)$ & n.s. \\
\hline
\end{tabular}


Influence of ventral tegmental area input on cortico-subcortical networks underlying action control and decision making

\begin{tabular}{|c|c|c|c|c|}
\hline $\begin{array}{l}\mathrm{R} \text { posterior } \\
\text { frontomedian } \\
\text { cortex }\end{array}$ & n.s. & $91146(3.20)$ & $151134(2.85)$ & n.s. \\
\hline $\begin{array}{l}\text { L posterior } \\
\text { frontomedian } \\
\text { cortex }\end{array}$ & n.s. & $-12852(3.21)$ & $-12552(2.34)^{+}$ & n.s. \\
\hline L MCC & $-15834(2.38)^{+}$ & n.s. & n.s. & n.s. \\
\hline R PCC & $3-2234(2.15)^{+}$ & n.s. & n.s. & n.s. \\
\hline L PCC & $-12-1334(2.55)^{+}$ & n.s. & n.s. & n.s. \\
\hline $\begin{array}{l}\mathrm{R} \text { intraparietal } \\
\text { cortex } \\
\text { (descending } \\
\text { part) }\end{array}$ & $24-6449(2.63)$ & $27-5849(4.46)$ & $39-5246(2.41)^{+}$ & n.s. \\
\hline $\begin{array}{l}\mathrm{L} \text { intraparietal } \\
\text { cortex } \\
\text { (descending } \\
\text { part) }\end{array}$ & n.s. & $-33-4640(4.00)$ & $-36-5849(1.97)^{+}$ & n.s. \\
\hline $\mathbf{R}$ precuneus & $18-6755(2.82)$ & $12-6731(4.60)^{* *}$ & $15-6728(2.88)$ & n.s. \\
\hline $\mathrm{L}$ precuneus & $-12-5870(2.10)^{+}$ & $-9-7349(4.03)$ & $-15-6728(2.37)^{+}$ & n.s. \\
\hline $\begin{array}{l}\mathrm{R} \text { inferior } \\
\text { parietal lobule }\end{array}$ & $39-3434(2.91)$ & n.s. & n.s. & n.s. \\
\hline $\begin{array}{l}\mathrm{L} \text { inferior } \\
\text { parietal lobule }\end{array}$ & $-39-2840(2.54)^{+}$ & $-48-3146(2.74)$ & $-33-4940(1.97)^{+}$ & n.s. \\
\hline $\begin{array}{l}\text { R posterior } \\
\text { superior } \\
\text { temporal gyrus }\end{array}$ & $60-4319(2.50)^{+}$ & n.s. & n.s. & n.s. \\
\hline $\begin{array}{l}\text { L anterior } \\
\text { superior } \\
\text { temporal gyrus }\end{array}$ & $-45-4-14(2.35)^{+}$ & n.s. & n.s. & n.s. \\
\hline $\begin{array}{l}\mathrm{R} \text { middle } \\
\text { temporal gyrus }\end{array}$ & n.s. & $51-55-14(3.59)$ & $63-43-14(2.06)^{+}$ & n.s. \\
\hline $\begin{array}{l}R \text { inferior } \\
\text { temporal gyrus }\end{array}$ & $42-13-23(2.18)^{+}$ & n.s. & n.s. & n.s. \\
\hline $\begin{array}{l}\text { R middle } \\
\text { occipital gyrus }\end{array}$ & $39-8210(4.74)^{* * *}$ & $45-764(3.80)$ & n.s. & n.s. \\
\hline $\begin{array}{l}\text { L middle } \\
\text { occipital gyrus }\end{array}$ & $-36-767(3.74)$ & $-36-8513(3.57)$ & n.s. & n.s. \\
\hline $\begin{array}{l}\text { R extrastriate } \\
\text { occipital cortex }\end{array}$ & $15-9413(5.41)^{* * *}$ & $12-857(6.90)^{* *}$ & n.s. & $18-10016(2.67)$ \\
\hline $\begin{array}{l}\text { L extrastriate } \\
\text { occipital cortex }\end{array}$ & $-9-9110(4.28)$ & $-6-884(5.92)^{* *}$ & n.s. & n.s. \\
\hline $\mathrm{L}$ amygdala & n.s. & $-15-1-14(2.47)^{+}$ & $-15-1-14(2.40)^{+}$ & n.s. \\
\hline R putamen & $272-8(2.40)^{+}$ & n.s. & n.s. & n.s. \\
\hline$R$ thalamus & $9-161(2.32)^{+}$ & n.s. & n.s. & $21-1013(2.08)^{+}$ \\
\hline$L$ thalamus & $-12-164(2.27)^{+}$ & $-6-13-2(2.52)^{+}$ & n.s. & n.s. \\
\hline $\begin{array}{l}\text { R globus } \\
\text { pallidus }\end{array}$ & n.s. & $-12-41(2.57)^{+}$ & n.s. & n.s. \\
\hline
\end{tabular}

Abbreviations: avPFC, anteroventral prefrontal cortex; IFJ, inferior frontal junction; L, left; MFG, middle frontal gyrus; MCC, middle cingulate cortex; n.s., not significant; OFC, orbitofrontal cortex; PCC, posterior cingulate cortex; R, right; SFG, superior frontal gyrus. 
Activations are reported at $\mathrm{p}<0.005$, uncorrected, with a minimum cluster size of 10 voxels; ${ }^{+} \mathrm{p}<0.05$, uncorrected, with a minimum cluster size of 10 voxels; ${ }^{*} \mathrm{p}<0.05$ FWE-corrected for small volume (for SVC we used spheres with a radius of 6 and $8 \mathrm{~mm}$, around the maxima reported by Diekhof and Gruber, 2010 and Diekhof et al., 2012b); **p<0.05 FWE-corrected (whole brain).

Table S3 Increased psychophysiological interactions of further saliency processing brain regions in the desire saliency contrast compared to the desire contrast (A) and the reason saliency contrast compared to the reason contrast $(B)$

\begin{tabular}{|c|c|c|c|c|c|c|c|c|}
\hline \multirow[t]{4}{*}{ Region } & \multicolumn{2}{|c|}{$\begin{array}{l}\text { Seed area } \\
\text { L OFC (-54 29 -5) }\end{array}$} & \multicolumn{2}{|c|}{$\begin{array}{l}\text { Seed area } \\
\text { L IFG (-54 29 1) }\end{array}$} & \multicolumn{2}{|c|}{$\begin{array}{l}\text { Seed area } \\
\text { R ACC (12 } 828)\end{array}$} & \multicolumn{2}{|c|}{$\begin{array}{l}\text { Seed area } \\
\text { L ACC (-18 } 11 \text { 25) }\end{array}$} \\
\hline & (A) & (B) & (A) & (B) & (A) & (B) & (A) & (B) \\
\hline & $\mathbf{s D C}>$ & sRC > & $\mathbf{s D C}>$ & sRC > & $\mathbf{s D C}>$ & sRC > & $\mathbf{s D C}>$ & sRC > \\
\hline & DC & RC & DC & RC & DC & RC & DC & RC \\
\hline \multicolumn{9}{|c|}{ MNI coordinates (t-value) } \\
\hline $\begin{array}{l}\text { R dorsal/ } \\
\text { ventral } \\
\text { Striatum }\end{array}$ & n.s. & n.s. & n.s. & $\begin{array}{l}1226-11 \\
(3.50)^{+}\end{array}$ & n.s. & n.s. & n.s. & n.s. \\
\hline $\begin{array}{l}\text { L dorsal/ } \\
\text { ventral } \\
\text { Striatum }\end{array}$ & n.s. & n.s. & n.s. & $\begin{array}{l}-929-2 \\
(3.46)^{+}\end{array}$ & n.s. & n.s. & $\begin{array}{l}-314-5 \\
(1.72)^{+}\end{array}$ & n.s. \\
\hline $\begin{array}{l}\mathbf{R} \\
\text { midbrain/ } \\
\text { VTA }\end{array}$ & n.s. & $\begin{array}{l}9-22-14 \\
(3.65)^{*}\end{array}$ & n.s. & $\begin{array}{l}9-19-14 \\
(3.90)^{*}\end{array}$ & n.s. & n.s. & $\begin{array}{l}3-19-26 \\
(2.16)^{+}\end{array}$ & n.s. \\
\hline $\begin{array}{l}\text { L } \\
\text { midbrain/ } \\
\text { VTA }\end{array}$ & $\begin{array}{l}-3-10- \\
14 \\
(2.17)^{+}\end{array}$ & $\begin{array}{l}-15-25- \\
11 \\
(2.21)^{+}\end{array}$ & $\begin{array}{l}-6-22- \\
26 \\
(2.05)^{+}\end{array}$ & $\begin{array}{l}-6-22-8 \\
(2.67)^{+}\end{array}$ & $\begin{array}{l}-3-31- \\
14(2.79)\end{array}$ & n.s. & n.s. & n.s. \\
\hline
\end{tabular}

Abbreviations: ACC, anterior cingulate cortex; IFG, inferior frontal gyrus; L, left; n.s., not significant; OFC, orbitofrontal cortex; R, right. Activations are reported at $\mathrm{p}<0.005$, uncorrected; ${ }^{+} \mathrm{p}<0.05$, uncorrected; ${ }^{*} \mathrm{p}<0.05$ FWE-corrected for small volume (for SVC we used spheres with a radius of $8 \mathrm{~mm}$, around the maxima reported by Diekhof and Gruber, 2010). 


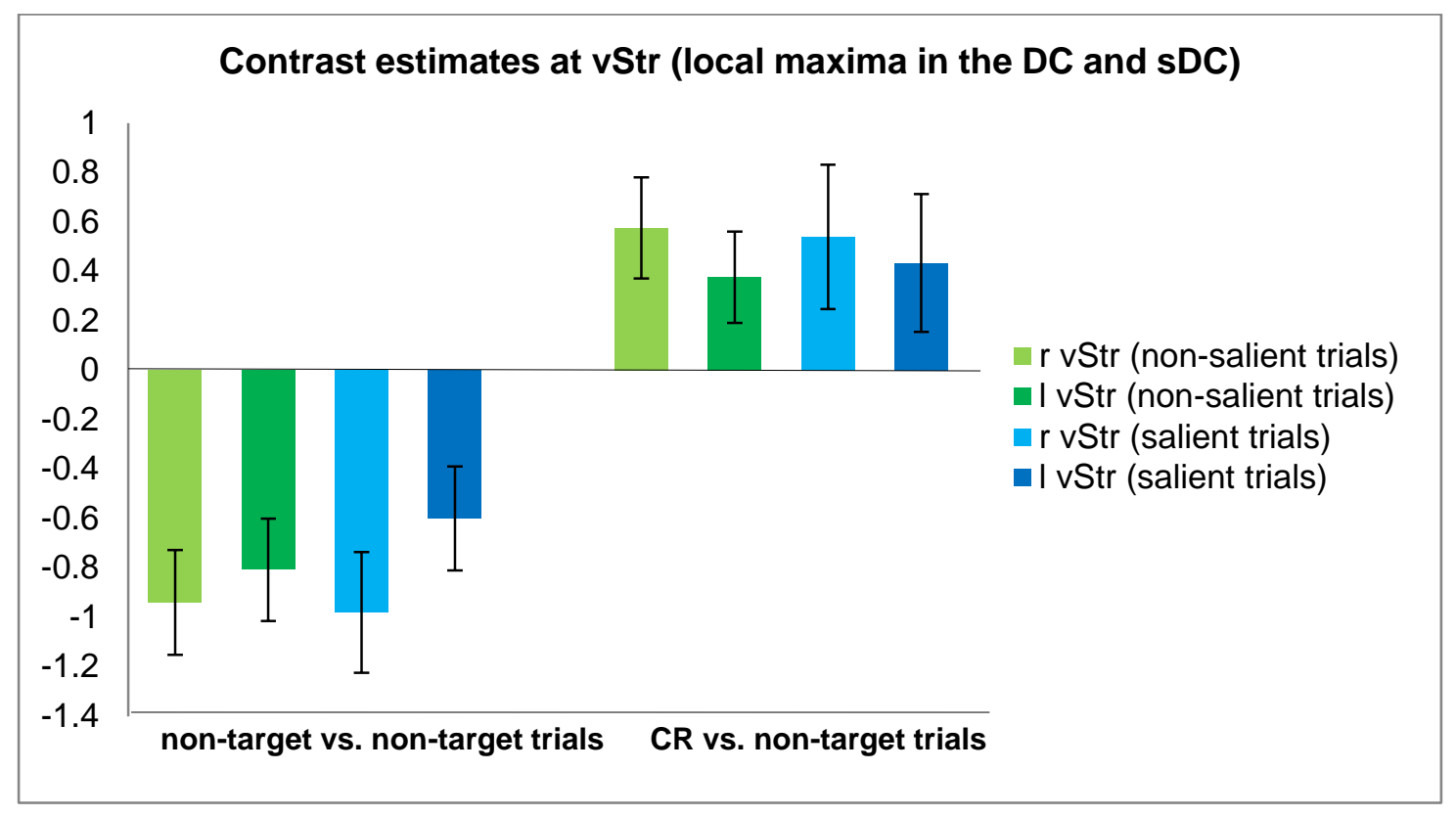

Figure S1. Contrast estimates for experimental trial types reflecting processing of saliency and reward. Contrast estimates at the vStr (mean \pm SEM). Individual local maxima for the right and left vStr were used from the desire contrast (DC) and the desire saliency contrast (sDC). Beta values were extracted from the following trial types: frequent non-target vs. non-target trials, infrequent and therefore salient non-target vs. non-target trials, frequent CR vs. non-target trials, infrequent and therefore salient CR vs. non-target trials. Regions listed in Table II. 


\section{General discussion}

\subsection{Summary of results}

In the present thesis I investigated the functioning of the mesolimbic dopamine system in order to address the main research questions namely how pathophysiological changes in schizophrenic patients affect behavior, functional activity and connectivity during reward processing, and how the experimental manipulation of salience affects the neural mechanisms involved in action control and reward-based decision making.

In the first study, patients with schizophrenia challenged with the DRD paradigm performed significantly worse in accepting and rejecting the reward stimuli independent of task context, and performed significantly worse in overall detecting the target stimuli when compared to healthy subjects. Successful target acceptance was necessary to achieve the superordinate task goal.

On the neural level, a comparison of both groups revealed significantly exaggerated neural responses of the vStr to the previously conditioned reward stimuli when they were allowed to accept in patients with schizophrenia, probably due to an intensified recruitment of this region during increased assignment of salience to these stimuli. This finding might appear to be contrary to previous studies investigating reward processing in schizophrenia and will be discussed later. However, the striatal hyperresponsivity in this thesis is in line with the subcortical hyperdopaminergic state in schizophrenia. Furthermore, the vStr hyperactivation was accompanied by an increase of activation in the middle frontal gyrus (MFG), inferior frontal junction (IFJ), frontal eye field (FEF) and intraparietal cortex, among other cortical brain regions (see Chapter 2, Table 2 and S1), which may reflect an inefficient attempt to compensate for the deficiency of these neural systems in schizophrenia. Contrary to this, significantly reduced reward-related brain activations were found in the right avPFC and left anterior MFG. This is in line with the divergent findings of frontal hypo- and hyperactivation in schizophrenia depending on working memory load and individual capacity limitations. Additionally, in the reason context where decisions counteracted immediate reward desiring, suppressed reward-related activation in the mesolimbic system was found in healthy participants. Group comparisons provided evidence for an attenuated suppression of reward signals in the vStr and VTA, accompanied by abnormal activation of several frontal and parietal brain regions in schizophrenia. Moreover, it has been shown that the reduced down-regulation of activation in the mesolimbic system was associated with an impaired functional connectivity between the vStr and both avPFC and VMPFC in schizophrenia, which is in line with the dysconnection hypothesis of schizophrenia (Friston, 1998). 
In the second study successful experimental implementation of saliency was confirmed by both behavioral and neuroimaging findings. Saliency was implemented by varying the incidence of the conditioned reward stimuli and neutral goal-irrelevant stimuli. Reaction times were significantly longer for trials where a target stimulus was presented simultaneously with an infrequent reward stimulus or an infrequent non-target stimulus compared to the equivalent trial type with a frequent reward stimulus/non-target stimulus. Regardless of the behavioral relevance of the infrequent stimuli, infrequency has been shown to exert a detrimental effect on performance. Furthermore, fMRI data revealed significantly increased activation of the vStr, VTA and further cortical brain regions such as the OFC, IFG and ACC during the presentation of infrequent neutral stimuli. This demonstrates that the mesolimbic reward system was activated in response to saliency per se and provided direct evidence for the role of the mesolimbic dopamine system in processing salient events in general. An increase of vStr and VTA activation has been further shown in response to both frequently and infrequently presented reward stimuli. Contrary to a previous hypothesis, comparison of infrequent to frequent reward trials revealed no further bottom-up activation, probably due to a BOLD ceiling effect. In the dilemma situation where actions required a restraint from immediate rewards, infrequent compared to frequent rewards led to an increased activation of the vStr and VTA, indicating a boosting of activation in both brain regions, probably caused by the salient and rewarding attributes of the stimuli. PPI analyses confirmed this assumption by uncovering an increased functional interaction between the VTA and vStr when the infrequent rewards compared to the frequent ones were presented in the desire and dilemma situation. Additionally, saliency modulated the interaction between different brain regions by increasing the functional connectivity between VTA and vStr and further saliency-processing regions including the OFC, amygdala, IFG and ACC, highlighting a network of various increased functional interactions within and beyond the mesolimbic reward system when processing salient events.

\subsection{Observed findings of reward circuitry dysfunctions in schizophrenia in the present thesis and their implications}

In view of the current research about schizophrenia and reward processing, essential differences in investigating the temporal aspects of processing rewards exist. A variety of studies either has examined neural correlates underlying reward anticipation, reward feedback or reward prediction error in schizophrenia. In the next section the findings of the present schizophrenia study will be discussed in the light of this variation. 
The hyperresponsivity of the vStr is consistent with the regionally specific subcortical hyperdopaminergic state in schizophrenia, proven by e.g. heightened levels of dopamine release (Abi-Dargham et al., 1998) and increased striatal dopamine synthesis capacity in the absence of incoming stimuli (e.g. for meta-analyses see Howes et al. 2012 and Fusar-Poli and MeyerLindenberg, 2013). The vStr and in particular the NAcc plays a central role in reward processing and in integrating widespread excitatory, inhibitory and modulatory afferents from cortical and limbic systems. The increased bottom-up activation of the vStr may result from an intensified recruitment of this region during exaggerated assignment of incentive salience to the conditioned reward stimuli. Kapur (2003) postulated that the aberrant attribution of motivational significance in schizophrenia occurred irrespective of changes in the context, therefore it is reasonable that the patient group was not able to flexibly adopt to the changing task types (DC, RC) leading to the hyperactivation of the vStr not only in the desire situation but also in the dilemma situation. Beyond that, I found reduced performance rates in the patient group in correctly accepting the conditioned reward stimuli in the desire situation and in correctly rejecting them in the dilemma situation in order to receive the maximal points. This is in accordance with proven deficits in delay-discounting tasks when choices between smaller immediate rewards and larger delayed rewards are required. Heerey et al. (2007) found that schizophrenic patients would choose a much smaller immediate reward over a larger delayed reward compared to healthy subjects. It is hypothesized that patients with schizophrenia fail to consider the possibility of losses when making decisions (Heerey et al., 2008). The observed deficits in the current thesis might reflect difficulties in integrating multiple features of a decision in the task as well as working memory capacity limitations. Moreover, schizophrenic patients have been previously shown to exhibit impaired functioning in rapid and reversal learning tasks, particularly in using negative feedback to flexibly alter previous rewarded responses (Waltz and Gold, 2007) whereas gradual learning seems to be intact (Morris et al., 2008). Overall, impairments in correctly rejecting the conditioned reward stimuli may be accounted for by deficits in reversal learning, learning new associations in probabilistic learning tasks and in the adaptation to changes in stimulus-reward contingencies, as this has been previously demonstrated in schizophrenic patients (Waltz and Gold, 2007; Weickert et al., 2009).

The observed hyperactivation of the vStr and also VTA in the desire and dilemma situation might appear to be contrary to previous studies investigating reward anticipation processes. The monetary incentive delay task similar to Knutson et al. (2001a) was mainly used in these studies. In the task subjects were confronted with cues that predicted monetary gain or loss. The outcome depended on their performance on a simple reaction time task at the end of each trial. This involved pressing a button during presentation of a visual target after a varying delay-period 
between cue and target. On the one hand, it has been shown that activation of dopaminergic brain areas during reward anticipation was elevated in schizophrenic patients similar to healthy controls (Abler et al., 2008). On the other hand, several fMRI studies using this task have found reduced vStr activation in response to the reward-indicating cues (e.g. Juckel et al., 2006a; Nielsen et al., 2012). These findings are in contrast to the observed increased activation of the vStr and VTA in this thesis. However, this is not surprising when taking into account that the monetary incentive delay task depends on the appropriate and intact functioning of anticipatory and related motivational processes. In contrast, in the present DRD paradigm participants had been conditioned to specific stimuli associated with a rewarding outcome in an operant conditioning task. This learning of stimulus-response-reward contingencies are based on vStr and VTA learning processes (Jimura et al., 2013). In this way, the paradigm allowed investigating the direct effects of reward stimuli without the need of anticipatory processes, and participants showed an immediate, automated bottom-up response of the dopaminergic reward system to the conditioned reward stimuli. It can therefore be concluded that disturbed anticipatory processes in schizophrenia led to vStr hypoactivation, whereas direct stimulation of the reward system is associated with a hyperactivation of the vStr.

Furthermore, numerous studies investigating reward feedback processing in schizophrenia have also used modified versions of a probabilistic monetary incentive delay task. Previous studies provided evidence that there were no significant differences between schizophrenic patients and healthy subjects in neural activation during receipt of a reward (Abler et al., 2008; Simon et al., 2010) and similar tracking of the valence and magnitude of outcomes in the vStr in patients and controls (Waltz et al., 2010). Additionally, Dowd and Barch (2012) used a passive Pavlovian reward prediction paradigm in which different presented cues varied in their predicted outcome. In this study they found largely intact brain responses to reward receipt in schizophrenic patients, while vStr and VMPFC activation during reward anticipation was reduced in patients with greater anhedonia severity. It is important to note, that the majority of the studies investigating reward receipt in schizophrenia used tasks where rewards were rather predictable and only relied on the correct reaction to a target stimulus. Hence, the main difference between these findings and the results of the present study might be the application of distinct paradigms investigating different processes.

Previous suggestions link aberrant salience to abnormal reward prediction error processing, leading to deficits in reinforcement learning in schizophrenia (for review see Heinz and Schlagenhauf, 2010). Although diverse neural measures have been assumed to reflect aberrant salience attribution in psychosis, most studies concentrated on the processing of reward-indicating cues. Thereby, aberrant salience is measured by response differences between reward-indicating and neutral cues. Morris et al. (2012) applied a reward-related prediction-error task to individuals 
with schizophrenia and found exaggerated neural responses in the vStr to expected rewards while responses to unexpected rewards in the vStr were severely attenuated. This bidirectional activation pattern suggests that the neural responses in the vStr of individuals with schizophrenia are aberrant and that they cannot appropriately differentiate between unexpected and expected events. The increased vStr response to expected rewards corresponds with the hyperactivation of the vStr to conditioned rewards in this thesis and is consistent with the assumption of persistent activity to well-predicted stimuli. Furthermore, functional activity was investigated in a classical passive Pavlovian conditioning task involving aversive events randomly mixed with neutral events. It has been demonstrated that individuals with schizophrenia exhibited a stronger response in the vStr and PFC to neutral events as compared to healthy controls. This evidence of aberrant attribution of salience led to aberrant learning, as shown by an inability to behaviorally distinguish between aversive and neutral stimuli, and was additionally confirmed by galvanic skin responses (Jensen et al., 2008). A previous study using an associative learning task observed an attenuation of the prediction-error-related signal in the PFC in schizophrenia (Corlett et al., 2007). Finally, Murray et al. (2008) applied an instrumental reward conditioning task and demonstrated reduced vStr and midbrain activation for reward-associated prediction errors compared with neutral prediction errors in schizophrenia.

Overall, by using differing paradigms, investigating different aspects of reward processing and varying stimulus or outcome uncertainty some studies found reduced reward-related activation (e.g. Nielsen et al., 2012), others described enhanced neural responses (e.g. Morris et al., 2012), and yet others reported no differences between schizophrenic patients and healthy controls during reward processing (Abler et al., 2008). By contributing to this still divergent current research, the presented findings highlight a pattern of exaggerated activation in the vStr among individuals with schizophrenia during reward-related decision-making.

Accumulating evidence indicates that it is not a dysfunction in the dopamine system itself that drives schizophrenia. Instead, disturbances in regulatory systems that exert afferent control over the dopamine system may contribute to the emergence of this disorder. Most notably deficits in the PFC and hippocampus have been shown to lead to impaired functioning of the dopamine system (see Grace, 2000 for review; Sesack and Carr, 2002).

Importantly, my thesis demonstrated for the first time that attenuated suppression of reward system activation in schizophrenic patients was associated with an impaired top-down control of reward signals in the vStr by prefrontal brain regions, in particular the avPFC and VMPFC. The role of the medial PFC in inhibitory top-down cognitive control has been well documented in various contexts, specifically fronto-striatal projections have been proposed to provide critical inhibitory control over the behavioral output of motivations (for a recently published study see 
Ferenczi et al., 2016). Prefronto-striatal interactions play an important role in the successful implementation of the task and disturbances may account for impairments in behavioral flexibility to achieve the long-term goal. Indeed, the neural finding of disturbed top-down control mechanisms was behaviorally expressed by reduced performance rates in correctly choosing the target color irrespective of the task context in the present thesis.

The impaired prefronto-striatal functional interaction of the current thesis is in line with the dysconnection hypothesis of schizophrenia (Friston, 1998; for review see Stephan et al., 2006) and the prior suggestion of fronto-striatal disruption in psychosis (see Robbins, 1990 for review). Support for this assumption already came from neuropsychological studies (e.g. Elliott et al., 1995; Hutton et al., 1998). A previous PET-study provided further evidence that prefrontal cortex dysfunction may be linked to dopaminergic transmission abnormalities in schizophrenia (MeyerLindenberg et al., 2002). Using resting-state fMRI, reduced connectivity between thalamus and prefrontal-striatal-cerebellar regions has been proven in schizophrenia which may reflect disturbances of sensory gating and top-down control (Anticevic et al., 2014). However, functional neuroimaging studies mainly applied working memory tasks when reporting diminished functional connectivity between PFC and substantia nigra in schizophrenic patients (Yoon et al., 2013) and fronto-striatal hypoactivation when correct information retrieval was necessary (Koch et al., 2008). One study has previously demonstrated abnormally larger connectivity strength from the vStr to the OFC in response to conditioned neutral stimuli compared to appetitive stimuli presented in an appetitive conditioning experiment (Diaconescu et al., 2011). Another study has shown reduced functional coupling between the medial PFC and the vStr during feedback processing in schizophrenia (Schlagenhauf et al., 2009). But it is worth noting that this study investigated activation during a feedback phase in an incentive delay task with gains and losses. I extend the knowledge about reward system dysfunction in schizophrenia by demonstrating a disturbed functional interaction between vStr and prefrontal brain regions during the presentation of conditioned reward stimuli in a reward-related decision making task where top-down control was needed.

Disturbances of functional coupling may be related to the reduction of density of mesoprefrontal dopamine fibers, as it has been revealed in postmortem schizophrenic brain (Akil et al., 1999). It is argued that a deficient control of synaptic plasticity manifests as abnormal functional integration of neural systems in schizophrenia (Stephan et al., 2006). In this thesis, however, it was not intended to identify the underlying cellular disturbances of the impaired functional connectivity in schizophrenic individuals by the use of fMRI. But it can be speculated that this finding results from impairments in synaptic transmission and plasticity. I also cannot rule out that the observed functional coupling might be due to shared interconnections with another area e.g. dopaminergic VTA afferents and further research is necessary to address this issue. 
Besides the displayed functional activation and connectivity abnormalities within the mesocorticolimbic dopamine system in schizophrenia, I also found abnormal neural responses in an extended fronto-parietal cortical network which has been shown to be involved in working memory, attention and cognitive control functions (see Gruber and Goschke, 2004 for review). In the following section I focus on the disparate findings of frontal activation patterns. For more information the interested reader may refer to Chapter 2, where I discuss the findings of the other brain regions in more detail.

Prefontal brain regions have been shown to be especially involved in working memory and executive functions (electrophysiological studies: Fuster et al., 1982; Funahashi et al., 1989; Chafee and Goldman-Rakic, 1998; fMRI studies: Manoach et al., 1997; D’Esposito et al., 2000). Moreover, many clinical and neuropsychological studies implicated prefrontal cortex dysfunction in schizophrenia (e.g. Goldman-Rakic, 1994; Pukrop et al., 2003; for a meta-analysis see Lee and Park, 2005). In the present study increased activation in response to the reward stimuli in the desire situation was found in the MFG. As opposed to this, decreased activation was observed in the avPFC and anterior parts of the MFG in schizophrenic patients. This is in line with the seemingly discrepant findings of previous studies. On the one hand, numerous neuroimaging studies of working memory provided evidence for task-related hypoactivation of prefrontal regions in schizophrenia (e.g. Andreasen et al., 1992; Barch et al., 2001; Menon et al., 2001). On the other hand, a number of studies demonstrated equal or increased prefrontal activity compared to healthy individuals (e.g. Manoach et al., 1999, 2000; Callicott et al., 2000). Based on these findings it must be emphasized that the reduced activation in prefrontal brain regions depends on the patients' symptoms and the complexity of the cognitive task employed. Overall, a number of variables are assumed to influence study outcome and contribute to the divergent activation findings. It is hypothesized that different working memory processes may be mediated by different subregions within prefrontal cortex (Manoach, 2003). Furthermore, it might be reasonably assumed that distinct deficits in specific subregions of PFC in patients with schizophrenia contribute to contrasting findings. Moreover, there is evidence that these activation differences also depend on working memory load and individual working memory capacity limitations in patients. Manoach (2003) proposed a hypothetical model illustrating how prefrontal brain activations within schizophrenic patients that are related to cognitive performance may vary as a function of both working memory load and capacity. It is hypothesized that frontal hyperactivation in schizophrenia might be a reflection of reduced working memory capacity and low to intermediate working memory load conditions as compared to healthy individuals. However, tasks requiring higher working memory performance, thus exceeding individual working memory capacities might elicit frontal hypoactivation in schizophrenic patients, reflecting that specific cognitive processes are more strongly involved (see Kircher and Gauggel, 
2008). The reported frontal hyperactivation in this thesis comply with earlier fMRI studies showing an increased response in the dorsolateral prefrontal cortex (DLPFC) in schizophrenia (Manoach, 1999, 2000). The hypoactivation of more anterior parts of frontal regions is in line with the assumption that prefrontal dysfunction in schizophrenia leads to impaired connectivity between prefrontal cortex and the mesolimbic system. Actually, a disturbed functional coupling between avPFC, VMPFC and the vStr has been shown in the present thesis. The bidirectional prefrontal activation pattern in this thesis lead to the assumption that these different subregions within the prefrontal cortex may have subserved different cognitive processes and may have been differently involved when challenged with the DRD paradigm causing both hyper- and hypoactivation.

Overall, increased activation of a widespread fronto-parietal network in this study probably may reflect an inefficient attempt to compensate for the deficiency of these neural systems subserving working memory, attentional selection and cognitive control processes. Alternatively, the abnormal activation pattern might be caused by an exaggerated attribution of motivational salience to the previously conditioned reward stimuli which may in turn lead to an increased recruitment of this network. Future studies investigating working memory processes in schizophrenia may benefit from a performance matching of the patient and control group and might concentrate on delineating the specific working memory processes and components that are impaired in schizophrenia.

\subsection{The mesolimbic dopamine system and its role in processing different forms of biologically significant events: reward and saliency}

Assuming that the described abnormal mesolimbic and fronto-parietal activation patterns in patients with schizophrenia reflect the aberrant intensified assignment of motivational significance to the conditioned reward stimuli, the question arose as to how generally salient events, rewarding or not, influence functional activity and connectivity within the mesolimbic dopamine system. To answer this question a modified paradigm was first applied to healthy individuals and it is intended to expand the observed findings and apply this paradigm to schizophrenic patients in the future. The novel design of the second study enabled the investigation of neural activation induced by saliency per se and in response to stimuli combining rewarding and salient attributes. Obviously, rewarding events themselves are salient due to their association with a reward. Though, saliency was implemented by the manipulation of both rewarding and neutral stimuli through relative frequency. Infrequent events are salient due to their oddball effect and have commonly been presumed to consistently elicit an orienting reflex. Although this is not the 
classical approach to investigate saliency as discussed below, behavioral and neuroimaging findings confirmed the successful experimental implementation of saliency in this thesis.

The previous assumption that behavioral performance is affected when infrequent and therefore salient stimuli compete with non-salient but behaviorally relevant stimuli for cognitive processing resources was endorsed by both increased reaction times and increased error rates in response to salient stimuli in general. In line with my findings, unexpected auditory pitch deviants that were behaviorally irrelevant led to significant longer reaction times and higher error rates in an auditory target-detection task (Sussman et al., 2003). Furthermore, previous studies reported prolonged reaction times for infrequent targets as compared to frequent standard events (Linden et al., 1999). Besides increased reaction times to infrequent targets, the same applies to responses to infrequent novels compared to frequently presented events (Kirino et al., 2000). This further led to the assumption that regardless of the behavioral relevance of the infrequent events, infrequency has been shown to exert a detrimental effect on performance. This is in line with the increased reaction times for target trials in the current study irrespective of whether the target stimulus was either presented together with an infrequent reward or an infrequent non-rewarding goal-irrelevant stimulus, which may be a result of readjustment in responses driven by conflict.

However, it could be argued that the increased response rates to trials comprising a target and infrequent reward when compared to the same trials including a frequent reward is rather related to learning differences in the operant conditioning of the two rewarded colors (red and green). Already in the beforehand operant conditioning task the red reward stimulus was presented six times less frequently than the green one. Existing studies reported that a stimulus needs to be presented increasingly often to be learned and to elicit an automatic response (for a detailed overview see Shiffrin and Schneider, 1977). In the present study a tradeoff was needed between presentation of infrequent reward stimuli and avoidance of fatigue because of a long-lasting experiment. Moreover, the duration of the operant conditioning task in the current study was based on previous implemented studies where the operant conditioning was successful and led to reliable bottom-up responses in the mesolimbic system. One major advantage of already manipulating the incidence of the stimuli in the conditioning phase was to avoid a "surprise effect", as this might have occurred when subjects would have been conditioned to rewards with the same incidence and afterwards in the fMRI experiment the rewards would have differed in their incidence. Nevertheless, by analyzing performance data of the conditioning task it turned out that there was no significant difference regarding incorrect responses to both frequently and infrequently presented rewarding colors (mean $( \pm$ SEM) of incorrect responses to non-salient rewards $=2.09 \pm 0.51$; mean $( \pm \mathrm{SEM})$ of incorrect responses to salient rewards $=1.26 \pm 0.24 ; \mathrm{t}_{(25)}=2.06$, $\mathrm{p}>0.05)$. 
On the neural level, infrequent neutral events led to a significantly increased activation of the VTA and vStr, providing direct evidence for the role of the mesolimbic system in processing salient events in general. The present paradigm required memory retrieval and alignment of significant information in terms of goal-relevance implemented by target stimuli, rewarding attributes and salient attributes of events. By varying the frequency of occurrence of the neutral stimuli, immediate orienting reactions to these attention attracting stimuli have been provoked. Hence, this activation pattern may be interpreted as representing a mechanism to detect and respond to potentially important changes in the sensory environment, as these infrequent stimuli may hold potentially significant information. The finding coincides well with a previous fMRI study where striatal activation has also been demonstrated in response to neutral events when they were unexpected (Zink et al., 2003). Thereby, salience was manipulated by altering the frequency of distractor occurrence in a visual target detection task in which NAcc activity increased when the distractors were both behaviorally relevant and irrelevant (Zink et al., 2003). However, earlier studies that have focused on salience processing commonly applied oddball paradigms to assess brain responses to rare target or distractor stimuli. There are diverging findings with reports of no striatal activity in a three-stimulus oddball task (Clark et al., 2000) and in a visual oddball target detection task (Kirino et al., 2000). However, a study using a cued task-switching paradigm was able to show that the VTA was activated when infrequent behaviorally relevant deviants where presented (Gruber et al., 2010). Beyond that, in the present study saliency per se activated a cortical network of brain areas involved in arousal, attentional reorienting and processing of visual salience, including OFC, IFG, insula, ACC, intraparietal and occipital cortex (Downar et al., 2000, 2001; Simmons et al., 2004; Huettel et al., 2005). Specifically, the OFC has been shown to be involved in the top-down facilitation of object recognition helping to rapidly detect motivationally significant stimuli in the environment (Bar et al., 2006, Bar, 2007). Furthermore, in previous oddball studies the intraparietal cortex has been observed to be activated in response to both attended targets and unattended distractors and insular activation was also shown in response to novel and infrequent deviant stimuli (Downar et al., 2001; Kiehl et al., 2005).

Overall, my findings extend prior research about saliency processing in classical oddball paradigms by highlighting that activation of both the VTA and vStr were significantly increased in response to infrequent neutral stimuli in a reward-related decision making paradigm.

Both frequently and infrequently presented events with a reward association represented a motivational goal and were probably perceived as highly significant. At the time of decision making the brain computes and compares the value and salient attributes of the stimuli. Value signals provide a measure of the desirability of these stimuli since they are associated with additional bonus points. Saliency signals provide a measure of the importance of the stimuli 
relating to motivational and attentional processes in the brain (for review see Rangel et al., 2008). In this context, correct and fast responses to these events enhanced the chance to gain a reward in addition to the total outcome. My findings showed that reward association exhibits a significant influence on subjects' performance irrespective of the manipulation of salience. Trials including a reward led to higher error rates when compared to trials without a reward. It is arguable that reward trials involve more cognitive control demands regarding recollection of information and alignment of the two simultaneously presented colors, especially in the case of a reward and target color. In every experimental run subjects had to memorize which of the two colors were presented as target color at the beginning of each run. These increased demands on cognitive control processes may give rise to the increased error rates.

The finding of salient rewards activating the vStr is consistent with earlier studies investigating brain regions underlying salience detection in a combinatory visual-auditory classification task (Zink et al., 2006) and during reward prediction (Jensen et al., 2007). Moreover, the findings of the present thesis extend prior research by demonstrating that not only the vStr but also the VTA were significantly activated by the interaction of valence and salience.

Against a former assumption, however, activation of the mesolimbic system showed no further increase in response to the infrequent rewards when compared to the frequently presented rewards. It might be speculated that an explanation for this pattern of results may derive from the so called BOLD ceiling effect (Birn and Bandettini, 2005). The ceiling effect is an important source of nonlinearity in the BOLD response and refers to the phenomenon in which a full oxygenation of hemoglobin has been reached so that even an infinite cerebral blood flow change could still produce only a finite BOLD response, corresponding to removing all deoxyhemoglobin from the voxel (Buxton et al., 2004). In the current study it is arguable that the neural activity of the mesolimbic reward system may be increased by both rewarding and salient attributes but the BOLD response was saturated. Extraction of beta values for the respective trial types supported this assumption (see Chapter 3, SI, Figure S1).

Recent findings provided clear evidence that dopamine neurons respond differently to highly diverse events (e.g. rewarding or aversive events), indicating that dopaminergic neurons are not homogeneous but rather divided into multiple subpopulations, each of them fulfilling distinct roles in motivational control (see Morales and Margolis, 2017). Electrophysiological animal studies (Matsumoto and Hikosaka, 2009; Matsumoto and Takada, 2013) have shown that one neuron population is excited by rewarding events and inhibited by aversive events, encoding motivational value. Hence, it is hypothesized that an appropriate instructive signal is provided by these neurons, engendering seeking, evaluation and value learning (Berridge, 2012). Another population has been observed to be excited by rewarding as well as aversive events in similar 
manners and that the responses to neutral events were weaker, as though encoding motivational salience. By this means, these neurons are assumed to provide signals to learn to adaptively respond to highly important situations, triggering orienting and explorative behavior (BrombergMartin et al., 2010). In the current thesis neither aversive stimuli nor omission of expected rewards were included in the task. Instead, saliency was implemented by variation of the frequency of occurrence of rewarding and neutral events. The majority of dopamine neurons have also been demonstrated to be excited in response to several types of sensory events irrespective of their rewarding or aversive attributes. These sensory events depend on novelty, frequency of occurrence, arousal and attention (Redgrave et al., 1999; Horvitz, 2000; Downar et al., 2002). By varying the frequency of occurrence of different stimuli, immediate orienting reactions to these attention attracting stimuli have been provoked. Bromberg-Martin et al. (2010) introduced a comprehensive model for distinct dopaminergic pathways and hypothesized that such sensory events generate an alerting signal. They further argued that these alerting signals may be assigned to motivational value- and salience-coding dopamine neurons and hence, may affect brain processing and behavior in a similar way to value and salience signals.

There were attempts to dissociate value and saliency signals at the time of decision making by the use of fMRI. Litt et al. (2011) applied a food choice task in which subjects were encouraged to make a decision about whether or not they wanted to eat the current food item at the end of the experiment. Afterwards they were presented with a picture of an item to give information about the strength of preference. In this way, they showed that the vStr was modulated by both value and saliency signals. This partly confirmed the finding of the present study although the implementation of salience varied widely since salience was given by the absolute value of the response (strong vs. low; Litt et al., 2011). In another variant of the monetary incentive delay task it was found that the vStr also correlates with both saliency and valence during the anticipation of probabilistic rewards (Cooper and Knutson, 2008). Again, however, saliency reposed on the variation of the certainty of the outcome, as anticipation of uncertain gains was argued to be more salient than certain losses.

In the current discussed study, due to the limited spatial resolution of fMRI it was improbable to separate the different neural activities relying on probably different midbrain neuron populations which may be in close vicinity to each other. Electrophysiological findings in monkeys further suggested that phasic and tonic activity of midbrain dopamine neurons code different aspects of reward information (e.g. Schultz et al., 1997). Taken these aspects into account, I conclude that the distinct value and salience mechanisms that probably occurred at different time scales may overlap in the current study. 
So far, studies investigating the functional connectivity during saliency processing are comparatively rare. The present thesis uncovered several increased functional interactions between the VTA and vStr as well as the OFC, IFG, amygdala and ACC during the presentation of infrequent rewards in the desire and dilemma situation (see Figure 2). It may be hypothesized that the salience-coding neurons in the VTA were strongly activated by infrequent rewards and conveyed significant information to the vStr leading to a boosting of activation in these brain regions when it was actually not favorable to choose the infrequent reward stimuli in the dilemma situation. My finding of a boosting of activation is contrary to previous studies demonstrating attenuated vStr and VTA activation in the dilemma situation which was related to a negative functional coupling between the vStr and prefrontal brain regions (Diekhof and Gruber, 2010; Diekhof et al., 2012a, b). Hence, it can be reasonably assumed that the saliency-modulated dopamine input from the VTA to the vStr may be stronger than inhibitory influences of the PFC and that the saliency signal in the VTA apparently was not suppressed by prefrontal regulatory mechanisms (Bromberg-Martin et al., 2010). Support for this assumption comes from previous studies which have shown that midbrain dopamine neurons project to the striatum, and in turn, that input to these neurons comes primarily from the striatum (Haber and Knutson, 2010). Projections from the striatum to the midbrain and again back to the striatum have been shown to create a loose topographic organization in primates (Haber et al., 2000). Significant information is hypothesized to be transferred between different functional regions of the striatum through midbrain dopamine neurons, thereby generating a feed forward organization from rewardassociated areas of the striatum to cognitive and motor regions. Especially the cortico-striatomidbrain pathway was thought to play an important role in influencing dopamine neurons and modifying responses to incoming significant and salient stimuli (Haber and Knutson, 2010).

Indeed, the present thesis also demonstrated increased functional couplings between VTA, vStr and the OFC. In addition, the vStr and amygdala also showed an increased functional interaction between each other. A previous human fMRI study also detected an increased functional interaction between the VTA and OFC when processing salient events in a cued task-switching paradigm (Diekhof et al., 2009). The findings of the thesis may be best interpreted in regard to a recent proposal (Horvitz, 2002), arguing that dopamine may not signal the motivationally significance of stimuli itself but rather may regulate orbitofrontal and amygdalar glutamatergic inputs to striatal regions, promoting reward-seeking behavior. The same applies to the input of corticostriatal sensory and motor signals which are necessary for the execution of correct responses. Both the OFC and amygdala are key regions in modulating the reward circuitry. The OFC has been shown to play a central role in processing the incentive and motivational value in animals (Schultz et al., 2000; Sesack and Grace, 2010). Furthermore, previous studies provided evidence that the amygdala was activated in response to all stimuli that were associated with high 
emotional salience independent of the valence (for review see Phan et al., 2004; Lewis et al., 2007). Both the OFC and the amygdala have been assumed to be of particular importance in the detection of general relevance of incoming biologically significant stimuli (Sander et al., 2003; Diekhof et al., 2011a). The constant monitoring and updating of sensory inputs for potential changes in stimulus significance is necessary to flexibly react to significant changes and to maximize the overall benefit. The infrequently presented rewarding stimuli represent such a change in stimulus significance and require allocation of attention processes (Posner and Petersen, 1990), filtering, sensory and behavioral orientation, motivation, action selection and execution (Redgrave et al., 2011). On this account, in the present study it seems convincing that both the OFC and the amygdala interacted with the key structures of the mesolimbic reward system when encoding motivational significant information which are necessary to guide goal-directed behavior. Moreover, in this complex network of multiple interactions an increased functional connectivity between the ACC and the VTA as well as vStr was observed in the present thesis. Both the dorsal ACC and the insula are known as key structures of the salience system (Lamichhane et al., 2016). Besides, the ACC was commonly observed in oddball processing and target detection tasks (Downar et al., 2001; Brázdil et al., 2005) and also in reversal learning studies (Kringelbach and Rolls, 2003). This highlights the important role of the ACC in significance and conflict processing.

Taken together, all these subcortical and cortical brain regions working in collaboration may form a network to ideally regulate selective attention and thus, to enable prioritized processing of salient stimulus attributes leading to adaptive and successful decision making. 


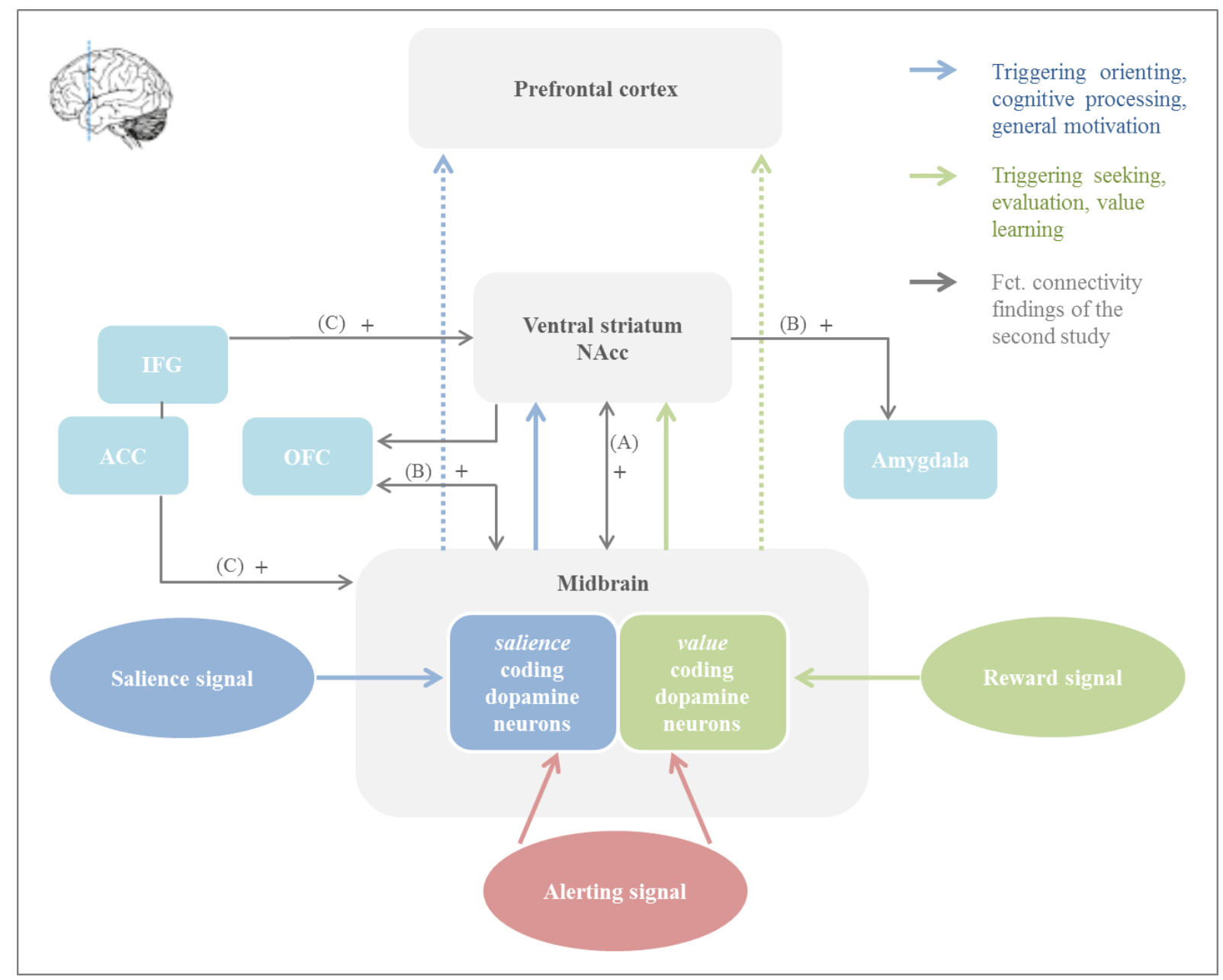

Figure 2. Hypothetical model of distinct dopamine neuron populations coding reward and salience within the mesocorticolimbic system.

This model combines aspects of a proposed model of Bromberg-Martin et al. (2010) (blue, green and red arrows) and the functional connectivity findings of the second study of the present thesis (dark gray arrows). It is supposed that motivational value signals are sent to value-coding dopamine neurons in the midbrain (green arrows), while motivational salience signals are sent to salience-coding dopamine neuron populations (blue arrows). Alerting signals are sent to both neuron groups (red arrows).

Moreover, PPI analyses of the second study using the VTA and vStr as seed regions revealed increased functional interactions (A) between each other and (B) between the VTA and vStr and the OFC and/or amygdala during the presentation of infrequent events in the desire and dilemma situation. Additionally, PPI analyses using the OFC, IFG and ACC as seed regions revealed (C) multiple increased functional interactions to the VTA and vStr, respectively. Important to note: direction of the dark gray arrows only reflects information about which of the brain regions were used as seed regions and which areas showed correlated activations. It does not provide evidence about the information flow between brain regions. More information regarding the detailed findings can be seen in Chapter 3 . 


\subsection{Relevance of the present thesis}

First, the present thesis was dedicated to investigating the mesocorticolimbic dopamine system during reward processing in individuals with schizophrenia in contrast to healthy subjects in order to contribute to a deeper understanding of the dysfunctional neural correlates of reward processing in the disorder. Second, the thesis was dedicated to examining how the brain processes biologically significant and salient events, in order to translate these findings into clinical research in the future (Figure 3).

First, the findings of the current thesis provide clear evidence for a hyperresponsivity of the vStr in schizophrenic patients in response to conditioned rewards irrespective of the task context. Therewith, this thesis gives significant insight into the way how immediate and automated mesolimbic responses to rewards were elicited, expanding the present knowledge about the pathophysiological changes in schizophrenia which was mainly based on the observation of reward-associated anticipatory or feedback-related processes. Moreover, the present results contribute to existing findings of functional connectivity by demonstrating disturbed functional interactions between the vStr and both the avPFC and VMPFC during reward-related decision making, and hence provide further evidence for the dysconnection hypothesis in schizophrenia (e.g. Stephan et al., 2006). Thus, these findings may contribute to a broader comprehension of bottom-up- and top-down-related reward mechanisms in the disorder.

Second, I could identify key structures such as the vStr, VTA, OFC, amygdala, IFG and ACC which are involved in the processing of salience and motivational significance. The findings of the present thesis clearly demonstrated vStr and VTA activation in response to neutral but infrequently presented events and also in response to rewarding events varying by their incidence. Therewith further support was provided for the assumed role of the mesolimbic dopamine system in processing salient events in general irrespective of their value. Furthermore, the present findings highlight the existence of a complex network of increased functional interactions between subcortical and cortical brain regions guiding adaptive processing of biologically significant events. These detected brain regions have been shown to be involved in relevance detection, coding motivational significance and reorienting attentional resources whenever significant environmental changes may occur (e.g. Horvitz, 2000; Downar et al., 2000, 2001; Schultz et al., 1998, 2000; Simmons et al., 2004; Ishikawa et al., 2008; Sesack and Grace, 2010). In sum, these functional activity and connectivity findings may provide a novel characterization of circuitry underlying interactions between the vStr, VTA, OFC, amygdala, IFG and ACC and provide new insight into the way how the modulation of salience affects functional activity and connectivity during decision making. 
The brain mechanisms and functional interactions underlying saliency processing in the healthy brain are of particular importance when these findings will be translated into clinical research. As the aberrant salience hypothesis in schizophrenia was previously introduced to explain the emergence of the disorder (Kapur, 2003), most studies investigated salience processing in schizophrenia based on the assumption that aberrant salience is linked to abnormal reward prediction processing. However, it is not yet clear which aspect of salience is the most critical one that is altered in psychosis (see Winton-Brown et al., 2014 for review). This thesis may reveal another approach to investigate neural correlates of motivational salience processing in patients with schizophrenia by the manipulation of relative frequency of neutral goal-irrelevant stimuli and stimuli which combines rewarding and salient attributes. Using this approach may provide new insight into aberrant salience processing in schizophrenia independent of prediction error processing and therefore may allow to specifically investigating the underlying potential dysfunctional neural mechanisms.

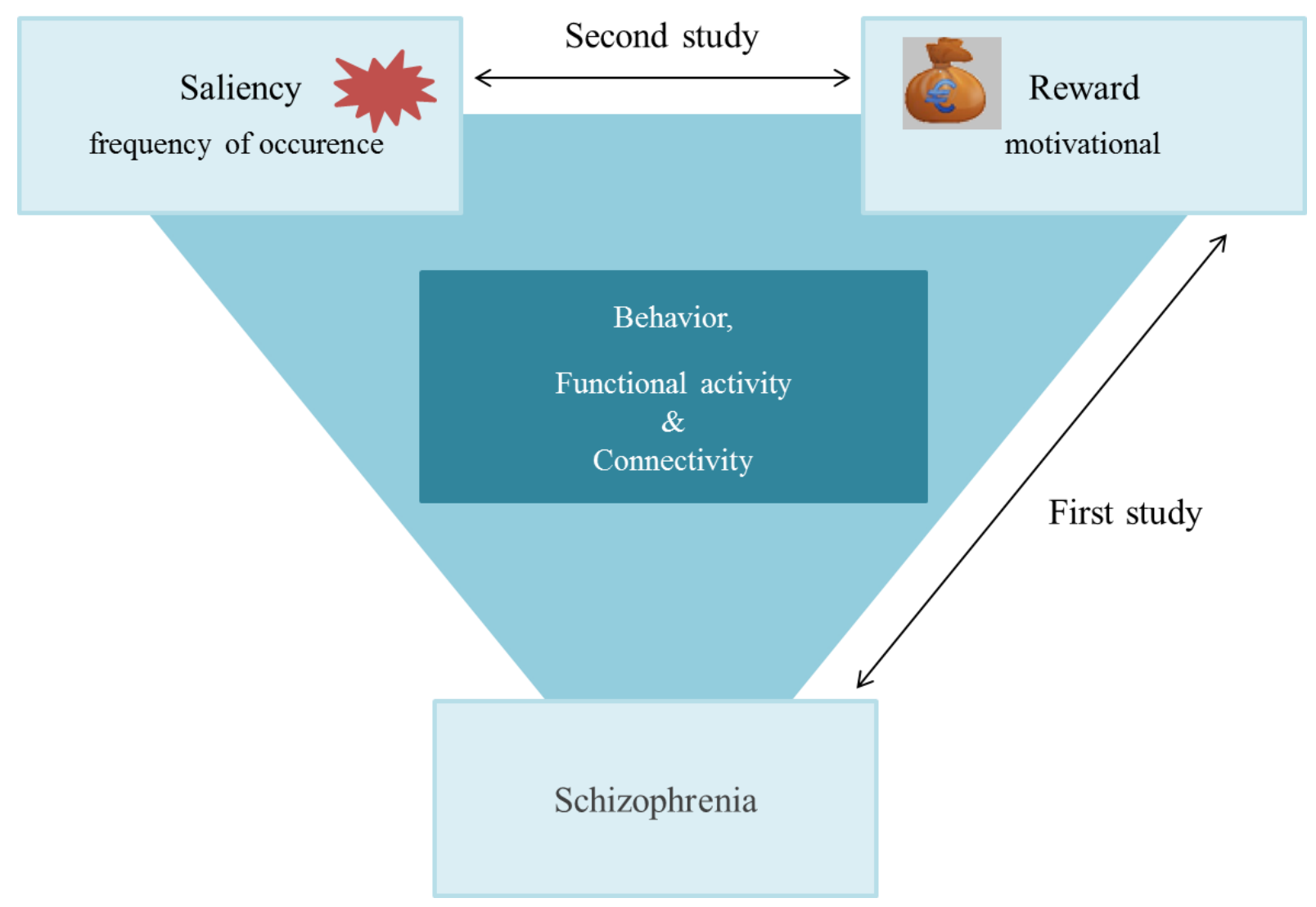

\section{Figure 3. Overview of the investigated mechanisms in this thesis.}

In the first study, I examined reward-related behavior as well as the functional activity and connectivity during reward processing in schizophrenia. In the second study, I investigated the influence of incidence of rewarding and neutral stimuli on the neural activation and functional connectivity in a reward-related decision making paradigm. 


\subsection{Limitations}

In the following section I would like to refer to some limitations and critical remarks on this thesis.

First, in the schizophrenia study all patients were medicated with typical or atypical drugs or both of them. Considering that these drugs are suggested to block dopaminergic pathways this may have an unintended impact on the presented data. However, it can be assumed that the increased reward signal observed in this thesis cannot be accounted for by antidopaminergic effects of antipsychotics because previous studies have demonstrated that typical or atypical antipsychotics led to normal patterns of VTA and vStr activation in schizophrenic patients (Abler et al., 2008). Moreover, neuroleptic-free schizophrenic patients exhibited increased presynaptic dopamine uptake capacity (Hietala et al., 1999; Lindström et al., 1999) and dopamine release (Abi-Dargham et al., 2000) leading to the conclusion that the observed hyperresponsivity of the mesolimbic system in the present study represent a stable neural correlate of the disease independent of medical treatment. Nevertheless, I cannot preclude that antipsychotic medication still has an impact on the observed findings. Patients were too ill to be scanned in a drug-free condition, so I was not able to address the question of whether the observed increased vStr activation was influenced by medication. Under ethical conditions it would be desirable to test at least a small group of unmedicated schizophrenic patients in the future to replicate these findings.

Second, one may note that the sample size of 16 schizophrenic patients is limited. However, this sample size is similar or even larger than the number of patients included in previous published studies providing significant results, such as Juckel et al. (2006a: $n=10)$, Abler et al. (2008: $n=12$ ) and Schlagenhauf et al. (2009: $n=15)$, which speaks in favor of sufficient statistical power.

Third, the included patients displayed both positive and negative symptoms. This might be the reason for the lack of a detected relationship between brain activation in a specific task context and symptom severity. It would be worthwhile to attempt to investigate subgroups of schizophrenic patients with prominent positive symptoms and in another group with negative symptoms to differentiate potential brain activations associated with individual symptoms.

Fourth, genetic and environmental factors might have an influence on the reported findings. Although it was not intended to investigate these factors in the present thesis, future studies should consider collecting these informations and regress or control for them in neuroimaging studies.

There are some methodological limitations regarding the second study that have to be mentioned. Due to the manipulation of incidence of reward stimuli in the modified paradigm the infrequent reward stimuli combined both rewarding and salient attributes. However, by the use of fMRI it 
was not possible to separate the underlying neural processes of value and saliency signals within the relatively small structures of the reward system. The study was not able to distinguish between different attentional, motor preparation or arousal signals due to the limited spatial resolution of the fMRI technique.

Furthermore, several confounders may have an impact on the reported findings. The difference between frequently and infrequently presented events may be accompanied by differences in stimulus familiarity which can influence attention. Previous experiences in making decisions for or against a stimulus can have an impact on the level of motor preparation, and in turn may affect overall levels of arousal. Also learning differences between the frequent and infrequent rewards as well as the infrequent neutral stimuli could have occurred. Although the duration of the operant conditioning task was based on previous studies where reliable bottom-up responses of the mesolimbic system have been reported and no significant differences regarding incorrect responses of both rewarding colors in the prior operant conditioning task could have been detected I cannot entirely preclude that the rare events were learned in a different manner. Potential learning differences could have resulted in different modes of information processing (see Shiffrin and Schneider, 1977). Events that have been well learned would require lower attention demands leading to automatic processing. In contrast, less well learned events that are less familiar would require controlled information processing which is much slower as compared to automatic processes. However, this argument would only apply to responses to infrequent rewards, as infrequent neutral events were not conditioned and present findings demonstrated reliable mesolimbic responses to these stimuli. In addition, it can be argued that by manipulating the incidence of specific stimuli varied in fact the predictability, as an infrequent stimulus is less expected. Overall, all these aspects might have an undesirable influence on the observed findings. Future research should pay more attention to these factors and control for them. Moreover, I would highly recommend to carefully proof the duration of reward conditioning to initially preclude potential confounds such as stimulus familiarity and predictability.

Besides, in order to introduce the factor saliency by means of relative frequency the applied paradigm became relatively complex. It was necessary to prolong the duration of the whole experiment to successfully implement infrequent events within a sequence of frequently presented events. This increase in the duration may have resulted in signs of fatigue and habituation to more often presented experimental conditions influencing neural processing and the measured BOLD signal. Furthermore, the observed neural effects in the studies partly did not survive whole-brain correction for multiple testing. Future experiments are needed to replicate these findings. 


\subsection{Conclusion and outlook}

The research that has been done within the scope of this thesis clearly revealed increased vStr activation in patients with schizophrenia when performing in a reward-based decision making task, consistent with the well-known subcortical hyperdopaminergic state in schizophrenia. Moreover, this thesis provided for the first time direct evidence for a disturbed top-down control of mesolimbic reward signals by prefrontal brain regions in schizophrenia, as proven by an impaired functional connectivity between the vStr and the avPFC as well as VMPFC. Consequently, these findings add to a growing body of literature concerning reward processing abnormalities in schizophrenia. However, the review of previous published research disclosed that recent findings are rather diverse. By applying various paradigms and investigating different aspects of reward processing (anticipation and feedback phase, prediction error processing), previous studies either reported reduced reward-related activation in schizophrenia or enhanced neural responses or rather a lack of activation differences between schizophrenic patients and healthy subjects. Therefore, it is of particular importance to identify and characterize behavioral and biological markers that are intrinsic to this complex disorder. As already described in the introduction of this thesis, schizophrenia is characterized by various altered behavioral and neural responses which are mediated by genetic, neurobiological and psychological processes. Future studies are needed to accurately examine trait markers representing the characteristics of behavioral and biological processes that are assumed to play a causal role in the pathophysiology of schizophrenia (Chen et al., 2006). Despite significant progress in this field it remains challenging to state which of the altered functional responses to rewards represent trait markers in schizophrenia. Identifying these markers may help to better understand the implicated pathomechanisms in schizophrenia.

A more comprehensive approach for future research would be to also raise genetic information as well as information about the environmental experience of adversity during childhood or lifetime for example. Such a combined analysis could account for reward-related functional activity or connectivity findings influenced by a particular genotype or by exposure of life-impairing experiences. Considering that dopamine has been shown to be strongly involved in schizophrenia, this thesis focused on the investigation of the dopaminergic reward system in schizophrenic individuals. Further research will be required to investigate interactions of dopamine with other neurotransmitters such as glutamate and to conclude whether abnormalities in neurotransmission of dopamine or glutamate are primary changes in the development of schizophrenia.

In the second study I demonstrated how the manipulation of salience through relative frequency modulated behavior as well as functional activity and connectivity within the reward circuitry. By 
showing that saliency per se led to an increase of activation, and in addition coding of infrequent rewards gave rise to a boosting of activation in the mesolimbic reward system, these findings contribute to the growing understanding of how brain mechanisms process and integrate the influence of salient and rewarding information on decision making. In future studies it would be interesting to examine how different salient attributes may be separately represented in various parts of the VTA and striatum taking into account the spatial and temporal aspects in processing salience. This could be achieved by the use of a higher spatial resolution of fMRI and a region-ofinterest approach focusing on the key regions of the mesolimbic system.

Furthermore, a significantly increased functional coupling between the VTA and vStr could be revealed. The presented findings also highlight the existence of multiple increased functional interactions between brain regions within and beyond the mesolimbic reward system underlying adaptive processing of salient events and successful behavioral decision making. The functional connectivity findings would benefit from further research making use of a dynamic causal modeling approach investigating the effective connectivity in order to draw conclusions about the information flow between the observed brain regions.

With regard to the aberrant salience hypothesis, a future study applying the modified saliency paradigm to patients with schizophrenia is of particular relevance to contribute to the understanding how infrequent rewarding events and infrequent neutral events are processed in schizophrenic patients. This modified paradigm would allow investigating disorder-specific disturbances regarding the functioning of the mesocorticolimbic system in the context of salience processing and motivation.

Altogether, the presented findings provide new insight into the functioning of the mesocorticolimbic system, into the extent of pathophysiological changes on behavior, functional activity and connectivity in schizophrenia and into the neural correlates of salience processing during reward-related decision making. 


\section{References}

Aalto, S., Ihalainen, J., Hirvonen, J., Kajander, J., Scheinin, H., Tanila, H., Nagren, K., Vilkman, H., Gustafsson, L. L., Syvälahti, E., \& Hietala, J. (2005). Cortical glutamate-dopamine interaction and ketamine-induced psychotic symptoms in man. Psychopharmacology, 182(3), 375-383. https://doi.org/10.1007/s00213-005-0092-6

Abi-Dargham, A., Gil, R., Krystal, J., Baldwin, R. M., Seibyl, J. P., Bowers, M., van Dyck, C. H., Charney, D. S., Innis, R. B., \& Laruelle, M. (1998). Increased striatal dopamine transmission in schizophrenia: confirmation in a second cohort. The American Journal of Psychiatry, 155(6), 761-767. https://doi.org/10.1176/ajp.155.6.761

Abi-Dargham, A., Rodenhiser, J., Printz, D., Zea-Ponce, Y., Gil, R., Kegeles, L. S., Weiss, R., Cooper, T. B., Mann, J. J., Van Heertum, R. L., Gorman, J. M., \& Laruelle, M. (2000). Increased baseline occupancy of D2 receptors by dopamine in schizophrenia. Proceedings of the National Academy of Sciences of the United States of America, 97(14), 8104-8109.

Abi-Dargham, A., Mawlawi, O., Lombardo, I., Gil, R., Martinez, D., Huang, Y., Hwang, D.-R., Keilp, J., Kochan, L., Van Heertum, R., Gorman, J. M., \& Laruelle, M. (2002). Prefrontal dopamine D1 receptors and working memory in schizophrenia. The Journal of Neuroscience: The Official Journal of the Society for Neuroscience, 22(9), 3708-3719. https://doi.org/20026302

Abi-Dargham, A., \& Moore, H. (2003). Prefrontal DA transmission at D1 receptors and the pathology of schizophrenia. The Neuroscientist: A Review Journal Bringing Neurobiology, Neurology and Psychiatry, 9(5), 404-416.

Abler, B., Walter, H., Erk, S., Kammerer, H., \& Spitzer, M. (2006). Prediction error as a linear function of reward probability is coded in human nucleus accumbens. NeuroImage, 31(2), 790-795. https://doi.org/10.1016/j.neuroimage.2006.01.001

Abler, B., Greenhouse, I., Ongur, D., Walter, H., \& Heckers, S. (2008). Abnormal reward system activation in mania. Neuropsychopharmacology: Official Publication of the American College of Neuropsychopharmacology, 33(9), 2217-2227. https://doi.org/10.1038/sj.npp.1301620

Akil, M., Pierri, J. N., Whitehead, R. E., Edgar, C. L., Mohila, C., Sampson, A. R., \& Lewis, D. A. (1999). Lamina-Specific Alterations in the Dopamine Innervation of the Prefrontal Cortex in Schizophrenic Subjects. American Journal of Psychiatry, 156(10), 1580-1589. https://doi.org/10.1176/ajp.156.10.1580

Alim, T. N., Lawson, W. B., Feder, A., Iacoviello, B. M., Saxena, S., Bailey, C. R., Greene, M. S., \& Neumeister, A. (2012). Resilience to meet the challenge of addiction: psychobiology and clinical considerations. Alcohol Research: Current Reviews, 34(4), 506-515. 
Ambroggi, F., Ishikawa, A., Fields, H. L., \& Nicola, S. M. (2008). Basolateral amygdala neurons facilitate reward-seeking behavior by exciting nucleus accumbens neurons. Neuron, 59(4), 648-661. https://doi.org/10.1016/j.neuron.2008.07.004

American Psychiatric Association (1994). Diagnostic and statistical manual of mental disorders. 4th edition. Washington: DSM-IV.

Andreasen, N. C., Rezai, K., Alliger, R., Swayze, V. W., Flaum, M., Kirchner, P., Cohen, G., \& O'Leary, D. S. (1992). Hypofrontality in neuroleptic-naive patients and in patients with chronic schizophrenia. Assessment with xenon 133 single-photon emission computed tomography and the Tower of London. Archives of General Psychiatry, 49(12), 943-958.

Andreasen, N. C., Arndt, S., Alliger, R., Miller, D., \& Flaum, M. (1995). Symptoms of schizophrenia. Methods, meanings, and mechanisms. Archives of General Psychiatry, 52(5), 341-351.

Anticevic, A., Cole, M. W., Repovs, G., Murray, J. D., Brumbaugh, M. S., Winkler, A. M., Savic, A., Krystal, J. H., Pearlson, G. D., \& Glahn, D. C. (2014). Characterizing thalamo-cortical disturbances in schizophrenia and bipolar illness. Cerebral Cortex (New York, N.Y.: 1991), 24(12), 3116-3130. https://doi.org/10.1093/cercor/bht165

Aosaki, T., Kimura, M., \& Graybiel, A. M. (1995). Temporal and spatial characteristics of tonically active neurons of the primate's striatum. Journal of Neurophysiology, 73(3), 12341252.

Arce, E., Leland, D. S., Miller, D. A., Simmons, A. N., Winternheimer, K. C., \& Paulus, M. P. (2006). Individuals with schizophrenia present hypo- and hyperactivation during implicit cueing in an inhibitory task. NeuroImage, 32(2), 704-713. https://doi.org/10.1016/j.neuroimage.2006.04.189

Avsar, K. B., Weller, R. E., Cox, J. E., Reid, M. A., White, D. M., \& Lahti, A. C. (2013). An fMRI investigation of delay discounting in patients with schizophrenia. Brain and Behavior, 3(4), 384-401. https://doi.org/10.1002/brb3.135

Ayalew, M., Le-Niculescu, H., Levey, D. F., Jain, N., Changala, B., Patel, S. D., Winiger, E., Breier, A., Shekhar, A., Amdur, R., Koller, D., Nurnberger, J. I., Corvin, A., Geyer, M., Tsuang, M. T., Salomon, D., Schork, N. J., Fanous, A. H., O’Donovan, M. C. \&Niculescu, A. B. (2012). Convergent functional genomics of schizophrenia: from comprehensive understanding to genetic risk prediction. Molecular Psychiatry, 17(9), 887-905. https://doi.org/10.1038/mp.2012.37

Bar, M., Kassam, K. S., Ghuman, A. S., Boshyan, J., Schmid, A. M., Dale, A. M., Hämäläinen, M. S., Marinkovic, K., Schacter, D. L., Rosen, B. R. \& Halgren, E. (2006). Top-down facilitation of visual recognition. Proceedings of the National Academy of Sciences of the United States of America, 103(2), 449-454. https://doi.org/10.1073/pnas.0507062103 
Bar, M. (2007). The proactive brain: using analogies and associations to generate predictions. Trends in Cognitive Sciences, 11(7), 280-289. https://doi.org/10.1016/j.tics.2007.05.005

Barch, D. M., Carter, C. S., Braver, T. S., Sabb, F. W., MacDonald, A., Noll, D. C., \& Cohen, J. D. (2001). Selective deficits in prefrontal cortex function in medication-naive patients with schizophrenia. Archives of General Psychiatry, 58(3), 280-288.

Barch, D. M., \& Dowd, E. C. (2010). Goal representations and motivational drive in schizophrenia: the role of prefrontal-striatal interactions. Schizophrenia Bulletin, 36(5), 919934. https://doi.org/10.1093/schbul/sbq068

Barnett, J. H., McDougall, F., Xu, M. K., Croudace, T. J., Richards, M., \& Jones, P. B. (2012). Childhood cognitive function and adult psychopathology: associations with psychotic and non-psychotic symptoms in the general population. The British Journal of Psychiatry: The Journal of Mental Science, 201, 124-130. https://doi.org/10.1192/bjp.bp.111.102053

Barrantes-Vidal, N., Aguilera, M., Campanera, S., Fatjó-Vilas, M., Guitart, M., Miret, S., Valero, S., \& Fañanás, L. (2007). Working memory in siblings of schizophrenia patients. Schizophrenia Research, 95(1-3), 70-75. https://doi.org/10.1016/j.schres.2007.06.020

Becerra, L., Breiter, H. C., Wise, R., Gonzalez, R. G., \& Borsook, D. (2001). Reward circuitry activation by noxious thermal stimuli. Neuron, 32(5), 927-946.

Bell, D. S. (1973). The Experimental Reproduction of Amphetamine Psychosis. Archives of General Psychiatry, 29(1), 35-40. https://doi.org/10.1001/archpsyc.1973.04200010020003

Bellgrove, M. A., Hester, R., \& Garavan, H. (2004). The functional neuroanatomical correlates of response variability: evidence from a response inhibition task. Neuropsychologia, 42(14), 1910-1916. https://doi.org/10.1016/j.neuropsychologia.2004.05.007

Benes, F. M. (2009). Neural circuitry models of schizophrenia: is it dopamine, GABA, glutamate, or something else? Biological Psychiatry, 65(12), 1003-1005. https://doi.org/10.1016/j.biopsych.2009.04.006

Bennett, M. R. (2011). Schizophrenia: susceptibility genes, dendritic-spine pathology and gray matter loss. Progress in Neurobiology, 95(3), 275-300. https://doi.org/10.1016/j.pneurobio.2011.08.003

Berns, G. S., McClure, S. M., Pagnoni, G., \& Montague, P. R. (2001). Predictability modulates human brain response to reward. The Journal of Neuroscience: The Official Journal of the Society for Neuroscience, 21(8), 2793-2798.

Berridge, K. C., \& Robinson, T. E. (1998). What is the role of dopamine in reward: hedonic impact, reward learning, or incentive salience? Brain Research. Brain Research Reviews, 28(3), 309-369. 
Berridge, K. C. (2007). The debate over dopamine's role in reward: the case for incentive salience. Psychopharmacology, 191(3), 391-431. https://doi.org/10.1007/s00213-006-0578$\mathrm{x}$

Berridge, K. C. (2012). From prediction error to incentive salience: mesolimbic computation of reward motivation. The European Journal of Neuroscience, 35(7), 1124-1143. https://doi.org/10.1111/j.1460-9568.2012.07990.x

Birn, R. M., \& Bandettini, P. A. (2005). The effect of stimulus duty cycle and "off" duration on BOLD response linearity. NeuroImage, 27(1), 70-82. https://doi.org/10.1016/j.neuroimage.2005.03.040

Bozarth, M. A. (1994). Pleasure systems in the brain. In: Warburton, D. M. (ed) Pleasure: The politics and the reality. John Wiley \& Sons, New York, 5-14.

Braff, D. L., Geyer, M. A., Light, G. A., Sprock, J., Perry, W., Cadenhead, K. S., \& Swerdlow, N. R. (2001). Impact of prepulse characteristics on the detection of sensorimotor gating deficits in schizophrenia. Schizophrenia Research, 49(1-2), 171-178.

Braff, D. L., \& Freedman, R. (2002). Endophenotypes in studies of the genetics of schizophrenia. In: Davis, K. L., Charney, D.S., Coyle, J.T., Nemeroff, C. (eds.) Neuropsychopharmacology: The Fifth Generation of Progress. Lippincott Williams \& Wilkins, Philadelphia, 703-716.

Brázdil, M., Dobsík, M., Mikl, M., Hlustík, P., Daniel, P., Pazourková, M., Krupa, P., \& Rektor, I. (2005). Combined event-related fMRI and intracerebral ERP study of an auditory oddball task. NeuroImage, 26(1), 285-293. https://doi.org/10.1016/j.neuroimage.2005.01.051

Brett, M., Anton, J., Valabregue, R., Poline, J. B. (2002). Region of interest analysis using an SPM toolbox. Neuroimage 16[Suppl 1], 1141.

Bromberg-Martin, E. S., Matsumoto, M., \& Hikosaka, O. (2010). Dopamine in motivational control: rewarding, aversive, and alerting. Neuron, 68(5), 815-834. https://doi.org/10.1016/j.neuron.2010.11.022

Buchsbaum, M. S., Someya, T., Teng, C. Y., Abel, L., Chin, S., Najafi, A., Haier, R. J., Wu, J., \& Bunney, W. E. (1996). PET and MRI of the thalamus in never-medicated patients with schizophrenia. The American Journal of Psychiatry, 153(2), 191-199. https://doi.org/10.1176/ajp.153.2.191

Buxton, R. B., Uludağ, K., Dubowitz, D. J., \& Liu, T. T. (2004). Modeling the hemodynamic response to brain activation. NeuroImage, 23, Supplement 1, S220-S233. https://doi.org/10.1016/j.neuroimage.2004.07.013

Calkins, M. E., \& Iacono, W. G. (2000). Eye movement dysfunction in schizophrenia: a heritable characteristic for enhancing phenotype definition. American Journal of Medical Genetics, 97(1), 72-76. 
Callicott, J. H., Bertolino, A., Mattay, V. S., Langheim, F. J., Duyn, J., Coppola, R., Goldberg, T. E., \& Weinberger, D. R. (2000). Physiological dysfunction of the dorsolateral prefrontal cortex in schizophrenia revisited. Cerebral Cortex (New York, N.Y.: 1991), 10(11), 10781092.

Cannon, M., Caspi, A., Moffitt, T. E., Harrington, H., Taylor, A., Murray, R. M., \& Poulton, R. (2002). Evidence for early-childhood, pan-developmental impairment specific to schizophreniform disorder: results from a longitudinal birth cohort. Archives of General Psychiatry, 59(5), 449-456.

Cannon, T. D., \& Keller, M. C. (2006). Endophenotypes in the genetic analyses of mental disorders. Annual Review of Clinical Psychology, 2, 267-290. https://doi.org/10.1146/annurev.clinpsy.2.022305.095232

Carlsson, A., \& Lindqvist, M. (1963). EFFECT OF CHLORPROMAZINE OR HALOPERIDOL ON FORMATION OF 3METHOXYTYRAMINE AND NORMETANEPHRINE IN MOUSE BRAIN. Acta Pharmacologica Et Toxicologica, 20, 140-144.

Chafee, M. V., \& Goldman-Rakic, P. S. (1998). Matching patterns of activity in primate prefrontal area 8a and parietal area 7ip neurons during a spatial working memory task. Journal of Neurophysiology, 79(6), 2919-2940.

Chen, X., Wang, X., O’Neill, A. F., Walsh, D., \& Kendler, K. S. (2004). Variants in the catecholo-methyltransferase (COMT) gene are associated with schizophrenia in Irish high-density families. Molecular Psychiatry, 9(10), 962-967. https://doi.org/10.1038/sj.mp.4001519

Chen, Y., Bidwell, L. C., \& Norton, D. (2006). Trait vs. State Markers for Schizophrenia: Identification and Characterization through Visual Processes. Current psychiatry reviews, 2(4), 431-438. https://doi.org/10.2174/157340006778699729

Chumbley, J. R., Tobler, P. N., \& Fehr, E. (2014). Fatal attraction: ventral striatum predicts costly choice errors in humans. NeuroImage, 89, 1-9. https://doi.org/10.1016/j.neuroimage.2013.11.039

Clark, V. P., Fannon, S., Lai, S., Benson, R., \& Bauer, L. (2000). Responses to rare visual target and distractor stimuli using event-related fMRI. Journal of Neurophysiology, 83(5), 31333139.

Clarke, H. F., Cardinal, R. N., Rygula, R., Hong, Y. T., Fryer, T. D., Sawiak, S. J., Ferrari, V., Cockcroft, G., Aigbirhio, F. I., Robbins, T. W., \& Roberts, A. C. (2014). Orbitofrontal dopamine depletion upregulates caudate dopamine and alters behavior via changes in reinforcement sensitivity. The Journal of Neuroscience: The Official Journal of the Society for Neuroscience, 34(22), 7663-7676. https://doi.org/10.1523/JNEUROSCI.0718-14.2014

Collier, D. A., \& Li, T. (2003). The genetics of schizophrenia: glutamate not dopamine? European Journal of Pharmacology, 480(1-3), 177-184. 
Cooper, J. C., \& Knutson, B. (2008). Valence and salience contribute to nucleus accumbens activation. NeuroImage, 39(1), 538-547. https://doi.org/10.1016/j.neuroimage.2007.08.009

Corbetta, M., \& Shulman, G. L. (2002). Control of goal-directed and stimulus-driven attention in the brain. Nature Reviews. Neuroscience, 3(3), 201-215. https://doi.org/10.1038/nrn755

Corlett, P. R., Murray, G. K., Honey, G. D., Aitken, M. R. F., Shanks, D. R., Robbins, T. W., Bullmore, E. T., Dickinson, A., \& Fletcher, P. C. (2007). Disrupted prediction-error signal in psychosis: evidence for an associative account of delusions. Brain: A Journal of Neurology, 130(Pt 9), 2387-2400. https://doi.org/10.1093/brain/awm173

Craddock, N., O’Donovan, M. C., \& Owen, M. J. (2005). The genetics of schizophrenia and bipolar disorder: dissecting psychosis. Journal of Medical Genetics, 42(3), 193-204. https://doi.org/10.1136/jmg.2005.030718

Creese, I., Burt, D. R., \& Snyder, S. H. (1976). Dopamine receptor binding predicts clinical and pharmacological potencies of antischizophrenic drugs. Science (New York, N.Y.), 192(4238), 481-483.

Cromwell, H. C., \& Schultz, W. (2003). Effects of expectations for different reward magnitudes on neuronal activity in primate striatum. Journal of Neurophysiology, 89(5), 2823-2838. https://doi.org/10.1152/jn.01014.2002

Crow, T. J. (1980). Molecular pathology of schizophrenia: more than one disease process? British Medical Journal, 280(6207), 66-68.

Davis, K. L., Kahn, R. S., Ko, G., \& Davidson, M. (1991). Dopamine in schizophrenia: a review and reconceptualization. The American Journal of Psychiatry, 148(11), 1474-1486. https://doi.org/10.1176/ajp.148.11.1474

Derrfuss, J., Brass, M., \& von Cramon, D. Y. (2004). Cognitive control in the posterior frontolateral cortex: evidence from common activations in task coordination, interference control, and working memory. NeuroImage, 23(2), 604-612. https://doi.org/10.1016/j.neuroimage.2004.06.007

D’Esposito, M., Postle, B. R., \& Rypma, B. (2000). Prefrontal cortical contributions to working memory: evidence from event-related fMRI studies. Experimental Brain Research, 133(1), 3-11. https://doi.org/10.1007/s002210000395

Diaconescu, A. O., Jensen, J., Wang, H., Willeit, M., Menon, M., Kapur, S., \& McIntosh, A. R. (2011). Aberrant Effective Connectivity in Schizophrenia Patients during Appetitive

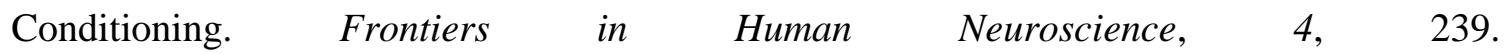
https://doi.org/10.3389/fnhum.2010.00239 
Diekhof, E. K., Falkai, P., \& Gruber, O. (2009). Functional interactions guiding adaptive processing of behavioral significance. Human Brain Mapping, 30(10), 3325-3331. https://doi.org/10.1002/hbm.20754

Diekhof, E. K., \& Gruber, O. (2010). When desire collides with reason: functional interactions between anteroventral prefrontal cortex and nucleus accumbens underlie the human ability to resist impulsive desires. The Journal of Neuroscience: The Official Journal of the Society for Neuroscience, 30(4), 1488-1493. https://doi.org/10.1523/JNEUROSCI.4690-09.2010

Diekhof, E. K., Falkai, P., \& Gruber, O. (2011a). The orbitofrontal cortex and its role in the assignment of behavioural significance. Neuropsychologia, 49(5), 984-991. https://doi.org/10.1016/j.neuropsychologia.2011.01.032

Diekhof, E. K., Geier, K., Falkai, P., \& Gruber, O. (2011b). Fear is only as deep as the mind allows: a coordinate-based meta-analysis of neuroimaging studies on the regulation of negative affect. Neurolmage, 58(1), 275-285. https://doi.org/10.1016/j.neuroimage.2011.05.073

Diekhof, E. K., Kaps, L., Falkai, P., \& Gruber, O. (2012a). The role of the human ventral striatum and the medial orbitofrontal cortex in the representation of reward magnitude - an activation likelihood estimation meta-analysis of neuroimaging studies of passive reward expectancy and outcome processing. Neuropsychologia, 50(7), 1252-1266. https://doi.org/10.1016/j.neuropsychologia.2012.02.007

Diekhof, E. K., Keil, M., Obst, K. U., Henseler, I., Dechent, P., Falkai, P., \& Gruber, O. (2012b). A functional neuroimaging study assessing gender differences in the neural mechanisms underlying the ability to resist impulsive desires. Brain Research, 1473, 63-77. https://doi.org/10.1016/j.brainres.2012.07.010

Diekhof, E. K., Nerenberg, L., Falkai, P., Dechent, P., Baudewig, J., \& Gruber, O. (2012c). Impulsive personality and the ability to resist immediate reward: an fMRI study examining interindividual differences in the neural mechanisms underlying self-control. Human Brain Mapping, 33(12), 2768-2784. https://doi.org/10.1002/hbm.21398

Dowd, E. C., \& Barch, D. M. (2012). Pavlovian reward prediction and receipt in schizophrenia: $\begin{array}{lllll}\text { relationship to } \text { anhedonia. PloS } & \text { One, } 35622 .\end{array}$ https://doi.org/10.1371/journal.pone.0035622

Downar, J., Crawley, A. P., Mikulis, D. J., \& Davis, K. D. (2000). A multimodal cortical network for the detection of changes in the sensory environment. Nature Neuroscience, 3(3), 277283. https://doi.org/10.1038/72991

Downar, J., Crawley, A. P., Mikulis, D. J., \& Davis, K. D. (2001). The effect of task relevance on the cortical response to changes in visual and auditory stimuli: an event-related fMRI study. NeuroImage, 14(6), 1256-1267. https://doi.org/10.1006/nimg.2001.0946 
Downar, J., Crawley, A. P., Mikulis, D. J., \& Davis, K. D. (2002). A cortical network sensitive to stimulus salience in a neutral behavioral context across multiple sensory modalities. Journal of Neurophysiology, 87(1), 615-620.

Eklund, A., Nichols, T. E., \& Knutsson, H. (2016). Cluster failure: Why fMRI inferences for spatial extent have inflated false-positive rates. Proceedings of the National Academy of Sciences of the United States of America, 113(28), 7900-7905. https://doi.org/10.1073/pnas.1602413113

Elliott, R., McKenna, P. J., Robbins, T. W., \& Sahakian, B. J. (1995). Neuropsychological evidence for frontostriatal dysfunction in schizophrenia. Psychological Medicine, 25(3), 619-630.

Elliott, R., Newman, J. L., Longe, O. A., \& William Deakin, J. F. (2004). Instrumental responding for rewards is associated with enhanced neuronal response in subcortical reward systems. NeuroImage, 21(3), 984-990. https://doi.org/10.1016/j.neuroimage.2003.10.010

Ewing, S. G., \& Grace, A. A. (2013). Long-term high frequency deep brain stimulation of the nucleus accumbens drives time-dependent changes in functional connectivity in the rodent limbic system. Brain Stimulation, 6(3), 274-285. https://doi.org/10.1016/j.brs.2012.07.007

Ferenczi, E. A., Zalocusky, K. A., Liston, C., Grosenick, L., Warden, M. R., Amatya, D., Katovich, K., Mehta, H., Patenaude, B., Ramakrishnan, C., Kalanithi, P., Etkin, A., Knutson, B., Glover, G. H., \& Deisseroth, K. (2016). Prefrontal cortical regulation of brainwide circuit dynamics and reward-related behavior. Science, 351(6268), aac9698. https://doi.org/10.1126/science.aac9698

Freedman, R. (2003). Electrophysiological phenotypes. Methods in Molecular Medicine, 77, 215225.

Friston, P., Jezzard, P., Turner, R. (1994). Analysis of functional MRI time-series. Human Brain Mapping, 1, 153-171

Friston, K. J., Buechel, C., Fink, G. R., Morris, J., Rolls, E., \& Dolan, R. J. (1997). Psychophysiological and modulatory interactions in neuroimaging. NeuroImage, 6(3), 218 229. https://doi.org/10.1006/nimg.1997.0291

Friston, K. J. (1998). The disconnection hypothesis. Schizophrenia Research, 30(2), 115-125.

Funahashi, S., Bruce, C. J., \& Goldman-Rakic, P. S. (1989). Mnemonic coding of visual space in the monkey's dorsolateral prefrontal cortex. Journal of Neurophysiology, 61(2), 331-349.

Fusar-Poli, P., Perez, J., Broome, M., Borgwardt, S., Placentino, A., Caverzasi, E., Cortesi, M., Veggiotti, P., Politi, P., Barale, F., \& McGuire, P. (2007). Neurofunctional correlates of vulnerability to psychosis: a systematic review and meta-analysis. Neuroscience and Biobehavioral Reviews, 31(4), 465-484. https://doi.org/10.1016/j.neubiorev.2006.11.006 
Fusar-Poli, P., \& Meyer-Lindenberg, A. (2013). Striatal Presynaptic Dopamine in Schizophrenia, Part II: Meta-Analysis of [18F/11C]-DOPA PET Studies. Schizophrenia Bulletin, 39(1), 3342. https://doi.org/10.1093/schbul/sbr180

Fuster, J. M., Bauer, R. H., \& Jervey, J. P. (1982). Cellular discharge in the dorsolateral prefrontal cortex of the monkey in cognitive tasks. Experimental Neurology, 77(3), 679-694.

Genzel, L., Dresler, M., Cornu, M., Jäger, E., Konrad, B., Adamczyk, M., Friess, E., Steiger, A., Czisch, M., \& Goya-Maldonado, R. (2015). Medial prefrontal-hippocampal connectivity and motor memory consolidation in depression and schizophrenia. Biological Psychiatry, 77(2), 177-186. https://doi.org/10.1016/j.biopsych.2014.06.004

Giakoumaki, S. G., Roussos, P., Pallis, E. G., \& Bitsios, P. (2011). Sustained attention and working memory deficits follow a familial pattern in schizophrenia. Archives of Clinical Neuropsychology: The Official Journal of the National Academy of Neuropsychologists, 26(7), 687-695. https://doi.org/10.1093/arclin/acr060

Gibbons, A. S., Scarr, E., Boer, S., Money, T., Jeon, W.-J., Felder, C., \& Dean, B. (2013). Widespread decreases in cortical muscarinic receptors in a subset of people with schizophrenia. The International Journal of Neuropsychopharmacology, 16(1), 37-46. https://doi.org/10.1017/S1461145712000028

Goldberg, X., Fatjó-Vilas, M., Muñoz, M. J., Campanera, S., Miret, S., Miñano, M. J., Aguilera, M., Miralles, M. L., Navarro, M. E., Lázaro, L., Guitart, M., Barrantes-Vidal, N., \& Fañanás, L. (2012). Increased familiarity of intellectual deficits in early-onset schizophrenia spectrum disorders. The World Journal of Biological Psychiatry: The Official Journal of the World Federation of Societies of Biological Psychiatry, 13(7), 493-500. https://doi.org/10.3109/15622975.2011.585661

Goldman-Rakic, P. S. (1994). Working memory dysfunction in schizophrenia. The Journal of Neuropsychiatry and Clinical Neurosciences, 6(4), 348-357. https://doi.org/10.1176/jnp.6.4.348

Goldman-Rakic, P. S. (1999). The physiological approach: functional architecture of working memory and disordered cognition in schizophrenia. Biological Psychiatry, 46(5), 650-661.

Gottesman, I. I., \& Gould, T. D. (2003). The endophenotype concept in psychiatry: etymology and strategic intentions. The American Journal of Psychiatry, 160(4), 636-645. https://doi.org/10.1176/appi.ajp.160.4.636

Gottfried, J. A., O’Doherty, J., \& Dolan, R. J. (2002). Appetitive and aversive olfactory learning in humans studied using event-related functional magnetic resonance imaging. The Journal of Neuroscience: The Official Journal of the Society for Neuroscience, 22(24), 1082910837. 
Grace, A. A. (1991). Phasic versus tonic dopamine release and the modulation of dopamine system responsivity: a hypothesis for the etiology of schizophrenia. Neuroscience, 41(1), 124.

Grace, A. A. (2000). Gating of information flow within the limbic system and the pathophysiology of schizophrenia. Brain Research. Brain Research Reviews, 31(2-3), 330341.

Grace, A. A., Floresco, S. B., Goto, Y., \& Lodge, D. J. (2007). Regulation of firing of dopaminergic neurons and control of goal-directed behaviors. Trends in Neurosciences, 30(5), 220-227. https://doi.org/10.1016/j.tins.2007.03.003

Grace, A. A. (2016). Dysregulation of the dopamine system in the pathophysiology of schizophrenia and depression. Nature Reviews. Neuroscience, 17(8), 524-532. https://doi.org/10.1038/nrn.2016.57

Green, M. F. (1996). What are the functional consequences of neurocognitive deficits in schizophrenia? The American Journal of Psychiatry, 153(3), 321-330. https://doi.org/10.1176/ajp.153.3.321

Greenwood, T. A., Lazzeroni, L. C., Murray, S. S., Cadenhead, K. S., Calkins, M. E., Dobie, D. J., .. Braff, D. L. (2011). Analysis of 94 candidate genes and 12 endophenotypes for schizophrenia from the Consortium on the Genetics of Schizophrenia. The American Journal of Psychiatry, 168(9), 930-946. https://doi.org/10.1176/appi.ajp.2011.10050723

Grimm, O., Heinz, A., Walter, H., Kirsch, P., Erk, S., Haddad, L., Plichta, M. M., RomanczukSeiferth, N., Pöhland, L., Mohnke, S., Mühleisen, T. W., Mattheisen, M., Witt, S. H., Schäfer, A., Cichon, S., Nöthen, M., Rietschel, M., Tost, H. \& Meyer-Lindenberg, A. (2014). Striatal response to reward anticipation: evidence for a systems-level intermediate phenotype for schizophrenia. JAMA Psychiatry, 71(5), 531-539. https://doi.org/10.1001/jamapsychiatry.2014.9

Gruber, O., \& von Cramon, D. Y. (2003). The functional neuroanatomy of human working memory revisited. Evidence from 3-T fMRI studies using classical domain-specific interference tasks. NeuroImage, 19(3), 797-809.

Gruber, O., \& Goschke, T. (2004). Executive control emerging from dynamic interactions between brain systems mediating language, working memory and attentional processes. Acta Psychologica, 115(2-3), 105-121. https://doi.org/10.1016/j.actpsy.2003.12.003

Gruber, O., Melcher, T., Diekhof, E. K., Karch, S., Falkai, P., \& Goschke, T. (2009). Brain mechanisms associated with background monitoring of the environment for potentially significant sensory events. Brain and Cognition, 69(3), 559-564. https://doi.org/10.1016/j.bandc.2008.11.008 
Gruber, O., Diekhof, E. K., Kirchenbauer, L., \& Goschke, T. (2010). A neural system for evaluating the behavioural relevance of salient events outside the current focus of attention. Brain Research, 1351, 212-221. https://doi.org/10.1016/j.brainres.2010.06.056

Guitart-Masip, M., Huys, Q. J. M., Fuentemilla, L., Dayan, P., Duzel, E., \& Dolan, R. J. (2012). Go and no-go learning in reward and punishment: interactions between affect and effect. NeuroImage, 62(1), 154-166. https://doi.org/10.1016/j.neuroimage.2012.04.024

Guo, N., Hwang, D.-R., Lo, E.-S., Huang, Y.-Y., Laruelle, M., \& Abi-Dargham, A. (2003). Dopamine depletion and in vivo binding of PET D1 receptor radioligands: implications for imaging studies in schizophrenia. Neuropsychopharmacology: Official Publication of the American College of Neuropsychopharmacology, 28(9), 1703-1711. https://doi.org/10.1038/sj.npp.1300224

Gur, R. E., Calkins, M. E., Gur, R. C., Horan, W. P., Nuechterlein, K. H., Seidman, L. J., \& Stone, W. S. (2007). The Consortium on the Genetics of Schizophrenia: neurocognitive endophenotypes. Schizophrenia Bulletin, 33(1), 49-68. https://doi.org/10.1093/schbul/sbl055

Haber, S. N., Fudge, J. L., \& McFarland, N. R. (2000). Striatonigrostriatal pathways in primates form an ascending spiral from the shell to the dorsolateral striatum. The Journal of Neuroscience: The Official Journal of the Society for Neuroscience, 20(6), 2369-2382.

Haber, S. N., \& Knutson, B. (2010). The reward circuit: linking primate anatomy and human imaging. Neuropsychopharmacology: Official Publication of the American College of Neuropsychopharmacology, 35(1), 4-26. https://doi.org/10.1038/npp.2009.129

Haijma, S. V., Van Haren, N., Cahn, W., Koolschijn, P. C. M. P., Hulshoff Pol, H. E., \& Kahn, R. S. (2013). Brain volumes in schizophrenia: a meta-analysis in over 18000 subjects. Schizophrenia Bulletin, 39(5), 1129-1138. https://doi.org/10.1093/schbul/sbs118

Hall, J., Trent, S., Thomas, K. L., O’Donovan, M. C., \& Owen, M. J. (2015). Genetic risk for schizophrenia: convergence on synaptic pathways involved in plasticity. Biological Psychiatry, 77(1), 52-58. https://doi.org/10.1016/j.biopsych.2014.07.011

Haller, C. S., Padmanabhan, J. L., Lizano, P., Torous, J., \& Keshavan, M. (2014). Recent advances in understanding schizophrenia. F1000prime Reports, 6, 57. https://doi.org/10.12703/P6-57

Harrison, P. J., \& Weinberger, D. R. (2005). Schizophrenia genes, gene expression, and neuropathology: on the matter of their convergence. Molecular Psychiatry, 10(1), 40-68; image 5. https://doi.org/10.1038/sj.mp.4001558

Hatzipetros, T., \& Yamamoto, B. K. (2006). Dopaminergic and GABAergic modulation of glutamate release from rat subthalamic nucleus efferents to the substantia nigra. Brain Research, 1076(1), 60-67. 
Heerey, E. A., Robinson, B. M., McMahon, R. P., \& Gold, J. M. (2007). Delay discounting in schizophrenia. Cognitive Neuropsychiatry, 12(3), 213-221. https://doi.org/10.1080/13546800601005900

Heerey, E. A., Bell-Warren, K. R., \& Gold, J. M. (2008). Decision-making impairments in the context of intact reward sensitivity in schizophrenia. Biological Psychiatry, 64(1), 62-69. https://doi.org/10.1016/j.biopsych.2008.02.015

Heinz, A., \& Schlagenhauf, F. (2010). Dopaminergic Dysfunction in Schizophrenia: Salience Attribution Revisited. Schizophrenia Bulletin, 36(3), 472-485. https://doi.org/10.1093/schbul/sbq031

Hibar, D. P., Westlye, L. T., van Erp, T. G. M., Rasmussen, J., Leonardo, C. D., Faskowitz, J., ... Andreassen, O. A. (2016). Subcortical volumetric abnormalities in bipolar disorder. Molecular Psychiatry, 21(12), 1710-1716. https://doi.org/10.1038/mp.2015.227

Hietala, J., Syvälahti, E., Vuorio, K., Räkköläinen, V., Bergman, J., Haaparanta, M., Solin, O., Kuoppamäki, M., Kirvelä, O., Ruotsalainen, U., \& Ruotsalainen, U. (1995). Presynaptic dopamine function in striatum of neuroleptic-naive schizophrenic patients. Lancet (London, England), 346(8983), 1130-1131.

Hietala, J., Syvälahti, E., Vilkman, H., Vuorio, K., Räkköläinen, V., Bergman, J., Haaparanta, M., Solin, O., Kuoppamaki, M., Eronen, E., Ruotsalainen, U., \& Salokangas, R. K. (1999). Depressive symptoms and presynaptic dopamine function in neuroleptic-naive schizophrenia. Schizophrenia Research, 35(1), 41-50.

Hoge, R. D., \& Pike, G. B. (2002). Quantitative measurement using MRI. In : Jezzard, P., Matthews, P. M., Smith, S. (eds.) Functional MRI. An introduction to methods. Oxford University Press, Oxford, 159-174.

Horvitz, J. C., Stewart, T., \& Jacobs, B. L. (1997). Burst activity of ventral tegmental dopamine neurons is elicited by sensory stimuli in the awake cat. Brain Research, 759(2), 251-258.

Horvitz, J. C. (2000). Mesolimbocortical and nigrostriatal dopamine responses to salient nonreward events. Neuroscience, 96(4), 651-656.

Horvitz, J. C. (2002). Dopamine gating of glutamatergic sensorimotor and incentive motivational input signals to the striatum. Behavioural Brain Research, 137(1-2), 65-74.

Howes, O. D., Montgomery, A. J., Asselin, M.-C., Murray, R. M., Valli, I., Tabraham, P., Bramon-Bosch, E., Valmaggia, L., Johns, L., Broome, M., McGuire, P. K., \& Grasby, P. M. (2009). Elevated striatal dopamine function linked to prodromal signs of schizophrenia. Archives of $\quad$ General 13-20. https://doi.org/10.1001/archgenpsychiatry.2008.514 
Howes, O. D., \& Kapur, S. (2009). The dopamine hypothesis of schizophrenia: version III--the final common pathway. Schizophrenia Bulletin, 35(3), 549-562. https://doi.org/10.1093/schbul/sbp006

Howes, O. D., Kambeitz, J., Kim, E., Stahl, D., Slifstein, M., Abi-Dargham, A., \& Kapur, S. (2012). The Nature of Dopamine Dysfunction in Schizophrenia and What This Means for Treatment: Meta-analysis of Imaging Studies. Archives of General Psychiatry, 69(8), 776786. https://doi.org/10.1001/archgenpsychiatry.2012.169

Huettel, S. A., Song, A. W., \& McCarthy, G. (2005). Decisions under Uncertainty: Probabilistic Context Influences Activation of Prefrontal and Parietal Cortices. Journal of Neuroscience, 25(13), 3304-3311. https://doi.org/10.1523/JNEUROSCI.5070-04.2005

Huettel, S. A., Song, A. W., \& McCarthy, G. (2009). Functional magnetic resonance imaging. 2nd edn. Sinauer Associates, Sunderland.

Hutton, S. B., Puri, B. K., Duncan, L. J., Robbins, T. W., Barnes, T. R., \& Joyce, E. M. (1998). Executive function in first-episode schizophrenia. Psychological Medicine, 28(2), 463-473.

Huttunen, J., Heinimaa, M., Svirskis, T., Nyman, M., Kajander, J., Forsback, S., Solin, O., Ilonen, T., Korkeila, J., Ristkari, T., McGlashan, T., Salokangas, R. K. R., \& Hietala, J. (2008). Striatal dopamine synthesis in first-degree relatives of patients with schizophrenia. Biological Psychiatry, 63(1), 114-117. https://doi.org/10.1016/j.biopsych.2007.04.017

Ikemoto, S. (2007). Dopamine reward circuitry: two projection systems from the ventral midbrain to the nucleus accumbens-olfactory tubercle complex. Brain Research Reviews, 56(1), 2778. https://doi.org/10.1016/j.brainresrev.2007.05.004

Ishikawa, A., Ambroggi, F., Nicola, S. M., \& Fields, H. L. (2008). Contributions of the amygdala and medial prefrontal cortex to incentive cue responding. Neuroscience, 155(3), 573-584. https://doi.org/10.1016/j.neuroscience.2008.06.037

Ito, R., Robbins, T. W., Pennartz, C. M., \& Everitt, B. J. (2008). Functional interaction between the hippocampus and nucleus accumbens shell is necessary for the acquisition of appetitive spatial context conditioning. The Journal of Neuroscience: The Official Journal of the Society for Neuroscience, 28(27), 6950-6959. https://doi.org/10.1523/JNEUROSCI.161508.2008

Jablensky, A. (2001). Symptoms of schizophrenia. In: Henn, F., Sartorius, N., Helmchen, H. et al. (eds.) Contemporary Psychiatry. Vol 3, Springer Verlag, Berlin, 6-36

Javitt, D. C. (2007). Glutamate and schizophrenia: phencyclidine, N-methyl-D-aspartate receptors, and dopamine-glutamate interactions. International Review of Neurobiology, 78, 69-108. https://doi.org/10.1016/S0074-7742(06)78003-5 
Jensen, J., Smith, A. J., Willeit, M., Crawley, A. P., Mikulis, D. J., Vitcu, I., \& Kapur, S. (2007). Separate brain regions code for salience vs. valence during reward prediction in humans. Human Brain Mapping, 28(4), 294-302. https://doi.org/10.1002/hbm.20274

Jensen, J., Willeit, M., Zipursky, R. B., Savina, I., Smith, A. J., Menon, M., Crawley, A. P., \& Kapur, S. (2008). The formation of abnormal associations in schizophrenia: neural and behavioral evidence. Neuropsychopharmacology: Official Publication of the American College of Neuropsychopharmacology, 33(3), 473-479. https://doi.org/10.1038/sj.npp.1301437

Jimura, K., Chushak, M. S., \& Braver, T. S. (2013). Impulsivity and Self-Control during Intertemporal Decision Making Linked to the Neural Dynamics of Reward Value Representation. Journal of Neuroscience, 33(1), 344-357. https://doi.org/10.1523/JNEUROSCI.0919-12.2013

Juckel, G., Schlagenhauf, F., Koslowski, M., Wüstenberg, T., Villringer, A., Knutson, B., Wrase, J., \& Heinz, A. (2006a). Dysfunction of ventral striatal reward prediction in schizophrenia. NeuroImage, 29(2), 409-416. https://doi.org/10.1016/j.neuroimage.2005.07.051

Juckel, G., Schlagenhauf, F., Koslowski, M., Filonov, D., Wüstenberg, T., Villringer, A., Knutson, B., Kienast, T., Gallinat, J., Wrase, J., \& Heinz, A. (2006b). Dysfunction of ventral striatal reward prediction in schizophrenic patients treated with typical, not atypical, neuroleptics. Psychopharmacology, 187(2), 222-228. https://doi.org/10.1007/s00213-0060405-4

Kapur, S. (2003). Psychosis as a state of aberrant salience: a framework linking biology, phenomenology, and pharmacology in schizophrenia. The American Journal of Psychiatry, 160(1), 13-23. https://doi.org/10.1176/appi.ajp.160.1.13

Kapur, S., Mizrahi, R., \& Li, M. (2005). From dopamine to salience to psychosis--linking biology, pharmacology and phenomenology of psychosis. Schizophrenia Research, 79(1), 59-68. https://doi.org/10.1016/j.schres.2005.01.003

Karam, C. S., Ballon, J. S., Bivens, N. M., Freyberg, Z., Girgis, R. R., Lizardi-Ortiz, J. E., Markx, S., Lieberman, J. A., \& Javitch, J. A. (2010). Signaling pathways in schizophrenia: emerging targets and therapeutic strategies. Trends in Pharmacological Sciences, 31(8), 381-390. https://doi.org/10.1016/j.tips.2010.05.004

Kendler, K. S., \& Neale, M. C. (2010). Endophenotype: a conceptual analysis. Molecular Psychiatry, 15(8), 789-797. https://doi.org/10.1038/mp.2010.8

Kestler, L. P., Walker, E., \& Vega, E. M. (2001). Dopamine receptors in the brains of schizophrenia patients: a meta-analysis of the findings. Behavioural Pharmacology, 12(5), 355-371. 
Kiehl, K. A., Stevens, M. C., Laurens, K. R., Pearlson, G., Calhoun, V. D., \& Liddle, P. F. (2005). An adaptive reflexive processing model of neurocognitive function: supporting evidence from a large scale $(\mathrm{n}=100)$ fMRI study of an auditory oddball task. NeuroImage, 25(3), 899-915. https://doi.org/10.1016/j.neuroimage.2004.12.035

Kircher, T. T. J., Liddle, P. F., Brammer, M. J., Williams, S. C. R., Murray, R. M., \& McGuire, P. K. (2002). Reversed lateralization of temporal activation during speech production in thought disordered patients with schizophrenia. Psychological Medicine, 32(3), 439-449.

Kircher, T., \& Gauggel, S. (2008). Neuropsychologie der Schizophrenie. Symptome, Kognition, Gehirn. 1st edn. Springer Medizin Verlag, Heidelberg, 243-251

Kirino, E., Belger, A., Goldman-Rakic, P., \& McCarthy, G. (2000). Prefrontal activation evoked by infrequent target and novel stimuli in a visual target detection task: an event-related functional magnetic resonance imaging study. The Journal of Neuroscience: The Official Journal of the Society for Neuroscience, 20(17), 6612-6618.

Kirkpatrick, B., Fenton, W. S., Carpenter, W. T., \& Marder, S. R. (2006). The NIMH-MATRICS consensus statement on negative symptoms. Schizophrenia Bulletin, 32(2), 214-219. https://doi.org/10.1093/schbul/sbj053

Kirov, G., O’Donovan, M. C., \& Owen, M. J. (2005). Finding schizophrenia genes. The Journal of Clinical Investigation, 115(6), 1440-1448. https://doi.org/10.1172/JCI24759

Knutson, B., Westdorp, A., Kaiser, E., \& Hommer, D. (2000). FMRI visualization of brain activity during a monetary incentive delay task. NeuroImage, 12(1), 20-27. https://doi.org/10.1006/nimg.2000.0593

Knutson, B., Adams, C. M., Fong, G. W., \& Hommer, D. (2001a). Anticipation of increasing monetary reward selectively recruits nucleus accumbens. The Journal of Neuroscience: The Official Journal of the Society for Neuroscience, 21(16), RC159.

Knutson, B., Fong, G. W., Adams, C. M., Varner, J. L., \& Hommer, D. (2001b). Dissociation of reward anticipation and outcome with event-related fMRI. Neuroreport, 12(17), 3683-3687.

Knutson, B., Fong, G. W., Bennett, S. M., Adams, C. M., \& Hommer, D. (2003). A region of mesial prefrontal cortex tracks monetarily rewarding outcomes: characterization with rapid event-related fMRI. NeuroImage, 18(2), 263-272.

Knutson, B., Taylor, J., Kaufman, M., Peterson, R., \& Glover, G. (2005). Distributed neural representation of expected value. The Journal of Neuroscience: The Official Journal of the Society for Neuroscience, 25(19), 4806-4812. https://doi.org/10.1523/JNEUROSCI.064205.2005 
Knutson, B., \& Greer, S. M. (2008). Anticipatory affect: neural correlates and consequences for choice. Philosophical Transactions of the Royal Society of London. Series B, Biological Sciences, 363(1511), 3771-3786. https://doi.org/10.1098/rstb.2008.0155

Koch, K., Wagner, G., Nenadic, I., Schachtzabel, C., Schultz, C., Roebel, M., Reichenbach, J. R., Sauer, H., \& Schlösser, R. G. M. (2008). Fronto-striatal hypoactivation during correct information retrieval in patients with schizophrenia: An fMRI study. Neuroscience, 153(1), 54-62. https://doi.org/10.1016/j.neuroscience.2008.01.063

Koch, K., Schachtzabel, C., Wagner, G., Schikora, J., Schultz, C., Reichenbach, J. R., Sauer, H., \& Schlösser, R. G. M. (2010). Altered activation in association with reward-related trialand-error learning in patients with schizophrenia. NeuroImage, 50(1), 223-232. https://doi.org/10.1016/j.neuroimage.2009.12.031

Krämer, B., \& Gruber, O. (2015). Dynamic Amygdala Influences on the Fronto-Striatal Brain Mechanisms Involved in Self-Control of Impulsive Desires. Neuropsychobiology, 72(1), 37 45. https://doi.org/10.1159/000437436

Kringelbach, M. L., \& Rolls, E. T. (2003). Neural correlates of rapid reversal learning in a simple model of human social interaction. NeuroImage, 20(2), 1371-1383. https://doi.org/10.1016/S1053-8119(03)00393-8

Kringelbach, M. L., \& Rolls, E. T. (2004). The functional neuroanatomy of the human orbitofrontal cortex: evidence from neuroimaging and neuropsychology. Progress in Neurobiology, 72(5), 341-372. https://doi.org/10.1016/j.pneurobio.2004.03.006

Krystal, J. H., Karper, L. P., Seibyl, J. P., Freeman, G. K., Delaney, R., Bremner, J. D., Heninger, G. R., Bowers, M. B., Charney, D. S., \& Charney, D. S. (1994). Subanesthetic effects of the noncompetitive NMDA antagonist, ketamine, in humans. Psychotomimetic, perceptual, cognitive, and neuroendocrine responses. Archives of General Psychiatry, 51(3), 199-214.

Lamichhane, B., Adhikari, B. M., \& Dhamala, M. (2016). Salience Network Activity in Perceptual Decisions. Brain Connectivity, 6(7), 558-571. https://doi.org/10.1089/brain.2015.0392

Laruelle, M. (1998). Imaging dopamine transmission in schizophrenia. A review and metaanalysis. The Quarterly Journal of Nuclear Medicine: Official Publication of the Italian Association of Nuclear Medicine (AIMN) [and] the International Association of Radiopharmacology (IAR), 42(3), 211-221.

Laruelle, M., \& Abi-Dargham, A. (1999). Dopamine as the wind of the psychotic fire: new evidence from brain imaging studies. Journal of Psychopharmacology (Oxford, England), 13(4), 358-371. 
Lauder, J. M., \& Bloom, F. E. (1974). Ontogeny of monoamine neurons in the locus coeruleus, Raphe nuclei and substantia nigra of the rat. I. Cell differentiation. The Journal of Comparative Neurology, 155(4), 469-481. https://doi.org/10.1002/cne.901550407

Lawrie, S. M., Whalley, H., Kestelman, J. N., Abukmeil, S. S., Byrne, M., Hodges, A., Rimmington, J. E., Best, J. J. K., Owens, D. G. C. \& Johnstone, E. C. (1999). Magnetic resonance imaging of brain in people at high risk of developing schizophrenia. Lancet (London, England), 353(9146), 30-33. https://doi.org/10.1016/S0140-6736(98)06244-8

Lee, K. H., \& Williams, L. M. (2000). Eye movement dysfunction as a biological marker of risk for schizophrenia. The Australian and New Zealand Journal of Psychiatry, 34 Suppl, S91100.

Lee, J., \& Park, S. (2005). Working memory impairments in schizophrenia: a meta-analysis. Journal of Abnormal Psychology, 114(4), 599-611. https://doi.org/10.1037/0021843X.114.4.599

Leube, D. T., Rapp, A., Buchkremer, G., Bartels, M., Kircher, T. T. J., Erb, M., \& Grodd, W. (2003). Hippocampal dysfunction during episodic memory encoding in patients with schizophrenia-an fMRI study. Schizophrenia Research, 64(1), 83-85.

Lewis, P. A., Critchley, H. D., Rotshtein, P., \& Dolan, R. J. (2007). Neural Correlates of Processing Valence and Arousal in Affective Words. Cerebral Cortex, 17(3), 742-748. https://doi.org/10.1093/cercor/bhk024

Linden, D. E., Prvulovic, D., Formisano, E., Völlinger, M., Zanella, F. E., Goebel, R., \& Dierks, T. (1999). The functional neuroanatomy of target detection: an fMRI study of visual and auditory oddball tasks. Cerebral Cortex (New York, N.Y.: 1991), 9(8), 815-823.

Lindsley, C. W., Shipe, W. D., Wolkenberg, S. E., Theberge, C. R., Williams, D. L., Sur, C., \& Kinney, G. G. (2006). Progress towards validating the NMDA receptor hypofunction hypothesis of schizophrenia. Current Topics in Medicinal Chemistry, 6(8), 771-785.

Lindström, L. H., Gefvert, O., Hagberg, G., Lundberg, T., Bergström, M., Hartvig, P., \& Långström, B. (1999). Increased dopamine synthesis rate in medial prefrontal cortex and striatum in schizophrenia indicated by L-(beta-11C) DOPA and PET. Biological Psychiatry, 46(5), 681-688.

Lisman, J. E., Coyle, J. T., Green, R. W., Javitt, D. C., Benes, F. M., Heckers, S., \& Grace, A. A. (2008). Circuit-based framework for understanding neurotransmitter and risk gene interactions in schizophrenia. Trends in Neurosciences, 31(5), 234-242. https://doi.org/10.1016/j.tins.2008.02.005

Litt, A., Plassmann, H., Shiv, B., \& Rangel, A. (2011). Dissociating valuation and saliency signals during decision-making. Cerebral Cortex (New York, N.Y.: 1991), 21(1), 95-102. https://doi.org/10.1093/cercor/bhq065 
Logothetis, N. K., Pauls, J., Augath, M., Trinath, T., \& Oeltermann, A. (2001). Neurophysiological investigation of the basis of the fMRI signal. Nature, 412(6843), 150157. https://doi.org/10.1038/35084005

Macpherson, T., Morita, M., \& Hikida, T. (2014). Striatal direct and indirect pathways control $\begin{array}{lllll}\text { decision-making behavior. Frontiers in Psychology, 5, } 1301 . & \end{array}$ https://doi.org/10.3389/fpsyg.2014.01301

Maher, B. S., Reimers, M. A., Riley, B. P., \& Kendler, K. S. (2010). Allelic heterogeneity in genetic association meta-analysis: an application to DTNBP1 and schizophrenia. Human Heredity, 69(2), 71-79. https://doi.org/10.1159/000264445

Manoach, D. S., Schlaug, G., Siewert, B., Darby, D. G., Bly, B. M., Benfield, A., Edelman, R. R., \& Warach, S. (1997). Prefrontal cortex fMRI signal changes are correlated with working memory load. Neuroreport, 8(2), 545-549.

Manoach, D. S., Press, D. Z., Thangaraj, V., Searl, M. M., Goff, D. C., Halpern, E., Saper, C. B., \& Warach, S. (1999). Schizophrenic subjects activate dorsolateral prefrontal cortex during a working memory task, as measured by fMRI. Biological Psychiatry, 45(9), 1128-1137.

Manoach, D. S., Gollub, R. L., Benson, E. S., Searl, M. M., Goff, D. C., Halpern, E., Saper, C. B. \& Rauch, S. L. (2000). Schizophrenic subjects show aberrant fMRI activation of dorsolateral prefrontal cortex and basal ganglia during working memory performance. Biological Psychiatry, 48(2), 99-109.

Manoach, D. S. (2003). Prefrontal cortex dysfunction during working memory performance in schizophrenia: reconciling discrepant findings. Schizophrenia Research, 60(2-3), 285-298.

Matsumoto, M., \& Hikosaka, O. (2009). Two types of dopamine neuron distinctly convey positive and negative motivational signals. Nature, 459(7248), 837-841. https://doi.org/10.1038/nature08028

Matsumoto, M., \& Takada, M. (2013). Distinct representations of cognitive and motivational signals in midbrain dopamine neurons. Neuron, 79(5), 1011-1024. https://doi.org/10.1016/j.neuron.2013.07.002

Matthews, P. M. (2002). An introduction to functional magnetic resonance imaging of the brain. In : Jezzard, P., Matthews, P. M., Smith, S. (eds.) Functional MRI. An introduction to methods. Oxford University Press, Oxford, 3-34.

Matthysse, S. (1973). Antipsychotic drug actions: a clue to the neuropathology of schizophrenia? Federation Proceedings, 32(2), 200-205.

McClure, S. M., Laibson, D. I., Loewenstein, G., \& Cohen, J. D. (2004). Separate neural systems value immediate and delayed monetary rewards. Science (New York, N.Y.), 306(5695), 503507. https://doi.org/10.1126/science.1100907 
McGrath, J., Saha, S., Chant, D., \& Welham, J. (2008). Schizophrenia: a concise overview of incidence, prevalence, and mortality. Epidemiologic Reviews, 30, 67-76. https://doi.org/10.1093/epirev/mxn001

McGrath, J. J., \& Murray, R. M. (2011). Environmental risk factors for schizophrenia. In: Weinberger, D. R., Harrison, P.J. (eds.) Schizophrenia, 3rd edn. Wiley-Blackwell, Oxford, 269-288.

Menon, V., Anagnoson, R. T., Mathalon, D. H., Glover, G. H., \& Pfefferbaum, A. (2001). Functional Neuroanatomy of Auditory Working Memory in Schizophrenia: Relation to Positive and Negative Symptoms. NeuroImage, 13(3), 433-446. https://doi.org/10.1006/nimg.2000.0699

Meyer-Lindenberg, A., Miletich, R. S., Kohn, P. D., Esposito, G., Carson, R. E., Quarantelli, M., Weinberger, D. R., \& Berman, K. F. (2002). Reduced prefrontal activity predicts exaggerated striatal dopaminergic function in schizophrenia. Nature Neuroscience, 5(3), 267-271. https://doi.org/10.1038/nn804

Miller, D. W., \& Abercrombie, E. D. (1996). Effects of MK-801 on spontaneous and amphetamine-stimulated dopamine release in striatum measured with in vivo microdialysis in awake rats. Brain Research Bulletin, 40(1), 57-62.

Mirenowicz, J., \& Schultz, W. (1996). Preferential activation of midbrain dopamine neurons by appetitive rather than aversive stimuli. Nature, 379(6564), 449-451. https://doi.org/10.1038/379449a0

Morales, M., \& Margolis, E. B. (2017). Ventral tegmental area: cellular heterogeneity, connectivity and behaviour. Nature Reviews Neuroscience, 18(2), 73-85. https://doi.org/10.1038/nrn.2016.165

Morris, S. E., Heerey, E. A., Gold, J. M., \& Holroyd, C. B. (2008). Learning-related changes in brain activity following errors and performance feedback in schizophrenia. Schizophrenia Research, 99(1-3), 274-285. https://doi.org/10.1016/j.schres.2007.08.027

Morris, R. W., Vercammen, A., Lenroot, R., Moore, L., Langton, J. M., Short, B., Kulkarni, J., Curtis, J., O’Donnell, M., Weickert, C. S., \& Weickert, T. W. (2012). Disambiguating ventral striatum fMRI-related BOLD signal during reward prediction in schizophrenia. Molecular Psychiatry, 17(3), 235, 280-289. https://doi.org/10.1038/mp.2011.75

Munafò, M. R., Attwood, A. S., \& Flint, J. (2008). Neuregulin 1 genotype and schizophrenia. Schizophrenia Bulletin, 34(1), 9-12. https://doi.org/10.1093/schbul/sbm129

Murray, G. K., Corlett, P. R., Clark, L., Pessiglione, M., Blackwell, A. D., Honey, G., Jones, P. B., Bullmore, E. T., Robbins, T. W., \& Fletcher, P. C. (2008). Substantia nigra/ventral tegmental reward prediction error disruption in psychosis. Molecular Psychiatry, 13(3), 239, 267-276. https://doi.org/10.1038/sj.mp.4002058 
Nielsen, M. Ø., Rostrup, E., Wulff, S., Bak, N., Lublin, H., Kapur, S., \& Glenthøj, B. (2012). Alterations of the Brain Reward System in Antipsychotic Naïve Schizophrenia Patients. Biological Psychiatry, 71(10), 898-905. https://doi.org/10.1016/j.biopsych.2012.02.007

O’Doherty, J. P., Deichmann, R., Critchley, H. D., \& Dolan, R. J. (2002). Neural responses during anticipation of a primary taste reward. Neuron, 33(5), 815-826.

O’Doherty, J., Dayan, P., Schultz, J., Deichmann, R., Friston, K., \& Dolan, R. J. (2004). Dissociable roles of ventral and dorsal striatum in instrumental conditioning. Science (New York, N.Y.), 304(5669), 452-454. https://doi.org/10.1126/science.1094285

O'Doherty, J. P. (2004). Reward representations and reward-related learning in the human brain: insights from neuroimaging. Current Opinion in Neurobiology, 14(6), 769-776. https://doi.org/10.1016/j.conb.2004.10.016

O’Donnell, P., \& Grace, A. A. (1995). Synaptic interactions among excitatory afferents to nucleus accumbens neurons: hippocampal gating of prefrontal cortical input. The Journal of Neuroscience: The Official Journal of the Society for Neuroscience, 15(5 Pt 1), 3622-3639.

O’Donovan, M. C., Owen, M. J. (2011). Genetic association in schizophrenia. In: Weinberger, D. R., Harrison, P. J. (eds.) Schizophrenia, 3rd edn. Wiley-Blackwell, Oxford, 269-288.

Ogawa, S., Lee, T. M., Nayak, A. S., \& Glynn, P. (1990a). Oxygenation-sensitive contrast in magnetic resonance image of rodent brain at high magnetic fields. Magnetic Resonance in Medicine, 14(1), 68-78.

Ogawa, S., Lee, T. M., Kay, A. R., \& Tank, D. W. (1990b). Brain magnetic resonance imaging with contrast dependent on blood oxygenation. Proceedings of the National Academy of Sciences of the United States of America, 87(24), 9868-9872.

Olds, J., \& Milner, P. (1954). Positive reinforcement produced by electrical stimulation of septal area and other regions of rat brain. Journal of Comparative and Physiological Psychology, 47(6), 419-427.

Olney, J. W., \& Farber, N. B. (1995). Glutamate receptor dysfunction and schizophrenia. Archives of General Psychiatry, 52(12), 998-1007.

Olney, J. W., Newcomer, J. W., \& Farber, N. B. (1999). NMDA receptor hypofunction model of schizophrenia. Journal of Psychiatric Research, 33(6), 523-533.

Owen, M. J., O’Donovan, M., Gottesman, I. I. (2003). Psychiatric genetics and genomics. Oxford: Oxford University Press, 247-266.

Owens, S. F., Picchioni, M. M., Rijsdijk, F. V., Stahl, D., Vassos, E., Rodger, A. K., Collier, D. A., Murray, R. M., \& Toulopoulou, T. (2011a). Genetic overlap between episodic memory 
deficits and schizophrenia: results from the Maudsley Twin Study. Psychological Medicine, 41(3), 521-532. https://doi.org/10.1017/S0033291710000942

Owens, S. F., Rijsdijk, F., Picchioni, M. M., Stahl, D., Nenadic, I., Murray, R. M., \& Toulopoulou, T. (2011b). Genetic overlap between schizophrenia and selective components of executive function. Schizophrenia Research, 127(1-3), 181-187. https://doi.org/10.1016/j.schres.2010.10.010

Pedersen, C. B., \& Mortensen, P. B. (2001). Evidence of a dose-response relationship between urbanicity during upbringing and schizophrenia risk. Archives of General Psychiatry, 58(11), 1039-1046.

Perälä, J., Suvisaari, J., Saarni, S. I., Kuoppasalmi, K., Isometsä, E., Pirkola, S., Partonen, T., Tuulio-Henriksson, A., Hintikka, J., Kieseppä, T., Härkänen, T., Koskinen, S., \& Lönnqvist, J. (2007). Lifetime prevalence of psychotic and bipolar I disorders in a general population. Archives of General Psychiatry, 64(1), 19-28. https://doi.org/10.1001/archpsyc.64.1.19

Perlstein, W. M., Carter, C. S., Noll, D. C., \& Cohen, J. D. (2001). Relation of prefrontal cortex dysfunction to working memory and symptoms in schizophrenia. The American Journal of Psychiatry, 158(7), 1105-1113. https://doi.org/10.1176/appi.ajp.158.7.1105

Perlstein, W. M., Dixit, N. K., Carter, C. S., Noll, D. C., \& Cohen, J. D. (2003). Prefrontal cortex dysfunction mediates deficits in working memory and prepotent responding in schizophrenia. Biological Psychiatry, 53(1), 25-38.

Phan, K. L., Wager, T. D., Taylor, S. F., \& Liberzon, I. (2004). Functional neuroimaging studies of human emotions. CNS Spectrums, 9(4), 258-266.

Pletnikov, M. V., Ayhan, Y., Nikolskaia, O., Xu, Y., Ovanesov, M. V., Huang, H., Mori, S., Moran, T. H., \& Ross, C. A. (2008). Inducible expression of mutant human DISC1 in mice is associated with brain and behavioral abnormalities reminiscent of schizophrenia. Molecular Psychiatry, 13(2), 173-186, 115. https://doi.org/10.1038/sj.mp.4002079

Poldrack, R. A., Mumford, J. A., Nichols, T. E. (2011). Handbook of functional MRI data analysis. 1st edn. Cambridge University Press, New York.

Posner, M. I., \& Petersen, S. E. (1990). The attention system of the human brain. Annual Review of Neuroscience, 13, 25-42. https://doi.org/10.1146/annurev.ne.13.030190.000325

Pukrop, R., Matuschek, E., Ruhrmann, S., Brockhaus-Dumke, A., Tendolkar, I., Bertsch, A., \& Klosterkötter, J. (2003). Dimensions of working memory dysfunction in schizophrenia. Schizophrenia Research, 62(3), 259-268.

Ragland, J. D., Gur, R. C., Glahn, D. C., Censits, D. M., Smith, R. J., Lazarev, M. G., Alavi, A., \& Gur, R. E. (1998). Frontotemporal cerebral blood flow change during executive and 
declarative memory tasks in schizophrenia: a positron emission tomography study. Neuropsychology, 12(3), 399-413.

Rangel, A., Camerer, C., \& Montague, P. R. (2008). A framework for studying the neurobiology of value-based decision making. Nature Reviews. Neuroscience, 9(7), 545-556. https://doi.org/10.1038/nrn2357

Rebec, G. V. (1998). Real-time assessments of dopamine function during behavior: single-unit recording, iontophoresis, and fast-scan cyclic voltammetry in awake, unrestrained rats. Alcoholism, Clinical and Experimental Research, 22(1), 32-40.

Redgrave, P., Prescott, T. J., \& Gurney, K. (1999). Is the short-latency dopamine response too short to signal reward error? Trends in Neurosciences, 22(4), 146-151.

Redgrave, P., Vautrelle, N., \& Reynolds, J. N. J. (2011). Functional properties of the basal ganglia's re-entrant loop architecture: selection and reinforcement. Neuroscience, 198, 138151. https://doi.org/10.1016/j.neuroscience.2011.07.060

Richter, A., Petrovic, A., Diekhof, E. K., Trost, S., Wolter, S., \& Gruber, O. (2015). Hyperresponsivity and impaired prefrontal control of the mesolimbic reward system in schizophrenia. Journal of Psychiatric Research, 71, 8-15. https://doi.org/10.1016/j.jpsychires.2015.09.005

Riehemann, S., Volz, H. P., Stützer, P., Smesny, S., Gaser, C., \& Sauer, H. (2001). Hypofrontality in neuroleptic-naive schizophrenic patients during the Wisconsin Card Sorting Test--a fMRI study. European Archives of Psychiatry and Clinical Neuroscience, 251(2), 66-71.

Robbins, T. W. (1990). The case of frontostriatal dysfunction in schizophrenia. Schizophrenia Bulletin, 16(3), 391-402.

Roiser, J. P., Stephan, K. E., den Ouden, H. E. M., Barnes, T. R. E., Friston, K. J., \& Joyce, E. M. (2009). Do patients with schizophrenia exhibit aberrant salience? Psychological Medicine, 39(2), 199-209. https://doi.org/10.1017/S0033291708003863

Roiser, J. P., Howes, O. D., Chaddock, C. A., Joyce, E. M., \& McGuire, P. (2013). Neural and behavioral correlates of aberrant salience in individuals at risk for psychosis. Schizophrenia Bulletin, 39(6), 1328-1336. https://doi.org/10.1093/schbul/sbs 147

Romaniuk, L., Honey, G. D., King, J. R. L., Whalley, H. C., McIntosh, A. M., Levita, L., Hughes, M., Johnstone, E. C., Day, M., Lawrie, S. M., \& Hall, J. (2010). Midbrain activation during Pavlovian conditioning and delusional symptoms in schizophrenia. Archives of General Psychiatry, 67(12), 1246-1254. https://doi.org/10.1001/archgenpsychiatry.2010.169

Sananbenesi, F., \& Fischer, A. (2009). The epigenetic bottleneck of neurodegenerative and psychiatric diseases. Biological Chemistry, 390(11), 1145-1153. https://doi.org/10.1515/BC.2009.131 
Sander, D., Grafman, J., \& Zalla, T. (2003). The human amygdala: an evolved system for relevance detection. Reviews in the Neurosciences, 14(4), 303-316.

Schizophrenia Working Group of the Psychiatric Genomics Consortium. (2014). Biological insights from 108 schizophrenia-associated genetic loci. Nature, 511(7510), 421-427. https://doi.org/10.1038/nature13595

Schlagenhauf, F., Juckel, G., Koslowski, M., Kahnt, T., Knutson, B., Dembler, T., Kienast, T., Gallinat, J., Wrase, J., \& Heinz, A. (2008). Reward system activation in schizophrenic patients switched from typical neuroleptics to olanzapine. Psychopharmacology, 196(4), 673-684. https://doi.org/10.1007/s00213-007-1016-4

Schlagenhauf, F., Sterzer, P., Schmack, K., Ballmaier, M., Rapp, M., Wrase, J., Juckel, G., Gallinat, J., \& Heinz, A. (2009). Reward feedback alterations in unmedicated schizophrenia patients: relevance for delusions. Biological Psychiatry, 65(12), 1032-1039. https://doi.org/10.1016/j.biopsych.2008.12.016

Schmaal, L., Hibar, D. P., Sämann, P. G., Hall, G. B., Baune, B. T., Jahanshad, N., ... Veltman, D. J. (2016a). Cortical abnormalities in adults and adolescents with major depression based on brain scans from 20 cohorts worldwide in the ENIGMA Major Depressive Disorder Working Group. Molecular Psychiatry. https://doi.org/10.1038/mp.2016.60

Schmaal, L., Veltman, D. J., van Erp, T. G. M., Sämann, P. G., Frodl, T., Jahanshad, N., ... Hibar, D. P. (2016b). Subcortical brain alterations in major depressive disorder: findings from the ENIGMA Major Depressive Disorder working group. Molecular Psychiatry, 21(6), 806-812. https://doi.org/10.1038/mp.2015.69

Schultz, W., Dayan, P., \& Montague, P. R. (1997). A neural substrate of prediction and reward. Science (New York, N.Y.), 275(5306), 1593-1599.

Schultz, W., Tremblay, L., \& Hollerman, J. R. (1998). Reward prediction in primate basal ganglia and frontal cortex. Neuropharmacology, 37(4-5), 421-429.

Schultz, W., Tremblay, L., \& Hollerman, J. R. (2000). Reward processing in primate orbitofrontal cortex and basal ganglia. Cerebral Cortex (New York, N.Y.: 1991), 10(3), 272-284.

Schumacher, J., Laje, G., Abou Jamra, R., Becker, T., Mühleisen, T. W., Vasilescu, C., Mattheisen, M., Herms, S., Hoffmann, P., Hillmer, A. M., Georgi, A., Herold, C., Schulze, T. G., Propping, P., Rietschel, M., McMahon, F. J., Nöthen, M. M., \& Cichon, S. (2009). The DISC locus and schizophrenia: evidence from an association study in a central European sample and from a meta-analysis across different European populations. Human Molecular Genetics, 18(14), 2719-2727. https://doi.org/10.1093/hmg/ddp204

Seeman, P., \& Lee, T. (1975). Antipsychotic drugs: direct correlation between clinical potency and presynaptic action on dopamine neurons. Science (New York, N.Y.), 188(4194), 12171219. 
Seeman, P., Lee, T., Chau-Wong, M., \& Wong, K. (1976). Antipsychotic drug doses and neuroleptic/dopamine receptors. Nature, 261(5562), 717-719.

Sesack, S. R., \& Carr, D. B. (2002). Selective prefrontal cortex inputs to dopamine cells: implications for schizophrenia. Physiology \& Behavior, 77(4-5), 513-517.

Sesack, S. R., \& Grace, A. A. (2010). Cortico-Basal Ganglia reward network: microcircuitry. Neuropsychopharmacology: Official Publication of the American College of Neuropsychopharmacology, 35(1), 27-47. https://doi.org/10.1038/npp.2009.93

Shiffrin, R. M., \& Schneider, W. (1977). Controlled and automatic human information processing: II. Perceptual learning, automatic attending, and a general theory. Psychological Review, 84, 127-189.

Shifman, S., Bronstein, M., Sternfeld, M., Pisanté, A., Weizman, A., Reznik, I., Spivak, B., Grisaru, N., Karp, L., Schiffer, R., Kotler, M., Strous, R. D., Swartz-Vanetik, M., Knobler, H. Y., Shinar, E., Yakir, B., Zak, N. B., \& Darvasi, A. (2004). COMT: a common susceptibility gene in bipolar disorder and schizophrenia. American Journal of Medical Genetics. Part B, Neuropsychiatric Genetics: The Official Publication of the International Society of Psychiatric Genetics, 128B(1), 61-64. https://doi.org/10.1002/ajmg.b.30032

Shizgal, P. (1997). Neural basis of utility estimation. Current Opinion in Neurobiology, 7(2), 198-208.

Simmons, A., Matthews, S. C., Stein, M. B., \& Paulus, M. P. (2004). Anticipation of emotionally aversive visual stimuli activates right insula. Neuroreport, 15(14), 2261-2265.

Simon, J. J., Biller, A., Walther, S., Roesch-Ely, D., Stippich, C., Weisbrod, M., \& Kaiser, S. (2010). Neural correlates of reward processing in schizophrenia--relationship to apathy and depression. Schizophrenia Research, 118(1-3), 154-161. https://doi.org/10.1016/j.schres.2009.11.007

Snyder, S. H. (1976). The dopamine hypothesis of schizophrenia: focus on the dopamine receptor. The American Journal of Psychiatry, 133(2), 197-202. https://doi.org/10.1176/ajp.133.2.197

Snyder, M. A., \& Gao, W.-J. (2013). NMDA hypofunction as a convergence point for progression and symptoms of schizophrenia. Frontiers in Cellular Neuroscience, 7, 31. https://doi.org/10.3389/fncel.2013.00031

Sommer, J. U., Schmitt, A., Heck, M., Schaeffer, E. L., Fendt, M., Zink, M., Nieselt K., Symons, S., Petroianu, G., Lex, A., Herrera-Marschitz, M., Spanagel, R., Falkai, P., \& GebickeHaerter, P. J. (2010). Differential expression of presynaptic genes in a rat model of postnatal hypoxia: relevance to schizophrenia. European Archives of Psychiatry and Clinical Neuroscience, 260 Suppl 2, S81-89. https://doi.org/10.1007/s00406-010-0159-1 
Stefansson, H., Sigurdsson, E., Steinthorsdottir, V., Bjornsdottir, S., Sigmundsson, T., Ghosh, S., ... Stefansson, K. (2002). Neuregulin 1 and susceptibility to schizophrenia. American Journal of Human Genetics, 71(4), 877-892. https://doi.org/10.1086/342734

Stefansson, H., Ophoff, R. A., Steinberg, S., Andreassen, O. A., Cichon, S., Rujescu, D., ... Collier, D. A. (2009). Common variants conferring risk of schizophrenia. Nature, 460(7256), 744-747. https://doi.org/10.1038/nature08186

Stephan, K. E., Baldeweg, T., \& Friston, K. J. (2006). Synaptic plasticity and dysconnection in $\begin{array}{lll}\text { schizophrenia. } & \text { Biological } & \text { Psychiatry, }\end{array}$ https://doi.org/10.1016/j.biopsych.2005.10.005

Stone, J. M., Morrison, P. D., \& Pilowsky, L. S. (2007). Glutamate and dopamine dysregulation in schizophrenia--a synthesis and selective review. Journal of Psychopharmacology (Oxford, England), 21(4), 440-452. https://doi.org/10.1177/0269881106073126

Straub, R. E., Jiang, Y., MacLean, C. J., Ma, Y., Webb, B. T., Myakishev, M. V., Harris-Kerr, C., Wormley, B., Sadek, H., Kadambi, B., Cesare, A. J., Gibberman, A., Wang, X., O’Neill, F. A., Walsh, D., \& Kendler, K. S. (2002). Genetic variation in the 6p22.3 gene DTNBP1, the human ortholog of the mouse dysbindin gene, is associated with schizophrenia. American Journal of Human Genetics, 71(2), 337-348. https://doi.org/10.1086/341750

Sussman, E., Sheridan, K., Kreuzer, J., \& Winkler, I. (2003). Representation of the standard: stimulus context effects on the process generating the mismatch negativity component of event-related brain potentials. Psychophysiology, 40(3), 465-471.

Sutton, R. S., \& Barto, A.G. (1998). Reinforcement learning: An introduction. MIT press Cambridge, 1312-1998.

Talkowski, M. E., Kirov, G., Bamne, M., Georgieva, L., Torres, G., Mansour, H., Chowdari, K. V., Milanova, V., Wood, J., McClain, L., Prasad, K., Shirts, B., Zhang, J., O’Donovan, M. C., Owen, M. J., Devlin, B. \& Nimgaonkar, V. L. (2008). A network of dopaminergic gene variations implicated as risk factors for schizophrenia. Human Molecular Genetics, 17(5), 747-758. https://doi.org/10.1093/hmg/ddm347

Tobler, P. N., O'doherty, J. P., Dolan, R. J., \& Schultz, W. (2006). Human neural learning depends on reward prediction errors in the blocking paradigm. Journal of Neurophysiology, 95(1), 301-310. https://doi.org/10.1152/jn.00762.2005

Toulopoulou, T., Picchioni, M., Rijsdijk, F., Hua-Hall, M., Ettinger, U., Sham, P., \& Murray, R. (2007). Substantial genetic overlap between neurocognition and schizophrenia: genetic modeling in twin samples. Archives of General Psychiatry, 64(12), 1348-1355. https://doi.org/10.1001/archpsyc.64.12.1348

Toulopoulou, T., Goldberg, T. E., Mesa, I. R., Picchioni, M., Rijsdijk, F., Stahl, D., Cherny, S. S., Sham, P., Faraone, S. V., Tsuang, M., Weinberger, D. R., Seidman, L. J., \& Murray, R. M. 
(2010). Impaired intellect and memory: a missing link between genetic risk and schizophrenia? Archives of General Psychiatry, 67(9), 905-913. https://doi.org/10.1001/archgenpsychiatry.2010.99

van Erp, T. G. M., Hibar, D. P., Rasmussen, J. M., Glahn, D. C., Pearlson, G. D., Andreassen, O. A., ... Turner, J. A. (2016). Subcortical brain volume abnormalities in 2028 individuals with schizophrenia and 2540 healthy controls via the ENIGMA consortium. Molecular Psychiatry, 21(4), 585. https://doi.org/10.1038/mp.2015.118

van Os, J., Rutten, B. P., \& Poulton, R. (2008). Gene-environment interactions in schizophrenia: review of epidemiological findings and future directions. Schizophrenia Bulletin, 34(6), 1066-1082. https://doi.org/10.1093/schbul/sbn117

Voineskos, A. N., Lerch, J. P., Felsky, D., Tiwari, A., Rajji, T. K., Miranda, D., Lobaugh, N. J., Pollock, B. G., Mulsant, B. H., \& Kennedy, J. L. (2011). The ZNF804A gene: characterization of a novel neural risk mechanism for the major psychoses. Neuropsychopharmacology: Official Publication of the American College of Neuropsychopharmacology, 36(9), 1871-1878. https://doi.org/10.1038/npp.2011.72

Vollenweider, F. X., Vontobel, P., Oye, I., Hell, D., \& Leenders, K. L. (2000). Effects of (S)ketamine on striatal dopamine: a [11C]raclopride PET study of a model psychosis in humans. Journal of Psychiatric Research, 34(1), 35-43.

Walter, H., Wunderlich, A. P., Blankenhorn, M., Schäfer, S., Tomczak, R., Spitzer, M., \& Grön, G. (2003). No hypofrontality, but absence of prefrontal lateralization comparing verbal and spatial working memory in schizophrenia. Schizophrenia Research, 61(2-3), 175-184.

Waltz, J. A., \& Gold, J. M. (2007). Probabilistic reversal learning impairments in schizophrenia: further evidence of orbitofrontal dysfunction. Schizophrenia Research, 93(1-3), 296-303. https://doi.org/10.1016/j.schres.2007.03.010

Waltz, J. A., Schweitzer, J. B., Gold, J. M., Kurup, P. K., Ross, T. J., Salmeron, B. J., Rose, E. J., McClure, S. M., \& Stein, E. A. (2009). Patients with schizophrenia have a reduced neural response to both unpredictable and predictable primary reinforcers. Neuropsychopharmacology: Official Publication of the American College of Neuropsychopharmacology, 34(6), 1567-1577. https://doi.org/10.1038/npp.2008.214

Waltz, J. A., Schweitzer, J. B., Ross, T. J., Kurup, P. K., Salmeron, B. J., Rose, E. J., Gold, J. M., \& Stein, E. A. (2010). Abnormal Responses to Monetary Outcomes in Cortex, but not in the Basal Ganglia, in Schizophrenia. Neuropsychopharmacology, 35(12), 2427-2439. https://doi.org/10.1038/npp.2010.126

Waltz, J. A., Frank, M. J., Wiecki, T. V., \& Gold, J. M. (2011). Altered probabilistic learning and response biases in schizophrenia: behavioral evidence and neurocomputational modeling. Neuropsychology, 25(1), 86-97. https://doi.org/10.1037/a0020882 
Watanabe, M. (1996). Reward expectancy in primate prefrontal neurons. Nature, 382(6592), 629632. https://doi.org/10.1038/382629a0

Weickert, T. W., Goldberg, T. E., Callicott, J. H., Chen, Q., Apud, J. A., Das, S., Zoltick, B. J., Egan, M. F., Meeter, M., Myers, C., Gluck, M. A., Weinberger, D. R., \& Mattay, V. S. (2009). Neural Correlates of Probabilistic Category Learning in Patients with Schizophrenia. Journal of Neuroscience, 29(4), 1244-1254. https://doi.org/10.1523/JNEUROSCI.434108.2009

Winton-Brown, T. T., Fusar-Poli, P., Ungless, M. A., \& Howes, O. D. (2014). Dopaminergic basis of salience dysregulation in psychosis. Trends in Neurosciences, 37(2), 85-94. https://doi.org/10.1016/j.tins.2013.11.003

Wise, R. A. (2004). Dopamine, learning and motivation. Nature Reviews. Neuroscience, 5(6), 483-494. https://doi.org/10.1038/nrn1406

Worsley, K. J., Marrett, S., Neelin, P., Vandal, A. C., Friston, K. J., \& Evans, A. C. (1996). A unified statistical approach for determining significant signals in images of cerebral activation. Human Brain Mapping, 4(1), 58-73. https://doi.org/10.1002/(SICI)10970193(1996)4:1<58::AID-HBM4>3.0.CO;2-O

Wright, I. C., Rabe-Hesketh, S., Woodruff, P. W., David, A. S., Murray, R. M., \& Bullmore, E. T. (2000). Meta-analysis of regional brain volumes in schizophrenia. The American Journal of Psychiatry, 157(1), 16-25. https://doi.org/10.1176/ajp.157.1.16

Yoon, J. H., Minzenberg, M. J., Raouf, S., D’Esposito, M., \& Carter, C. S. (2013). Impaired prefrontal-basal ganglia functional connectivity and substantia nigra hyperactivity in $\begin{array}{llll}\text { schizophrenia. } & \text { Biological } & \text { Psychiatry, } & \text { 74(2), }\end{array}$ https://doi.org/10.1016/j.biopsych.2012.11.018

Young, A. M., Joseph, M. H., \& Gray, J. A. (1992). Increased dopamine release in vivo in nucleus accumbens and caudate nucleus of the rat during drinking: a microdialysis study. Neuroscience, 48(4), 871-876.

Young, A. M., Joseph, M. H., \& Gray, J. A. (1993). Latent inhibition of conditioned dopamine release in rat nucleus accumbens. Neuroscience, 54(1), 5-9.

Zink, C. F., Pagnoni, G., Martin, M. E., Dhamala, M., \& Berns, G. S. (2003). Human striatal response to salient nonrewarding stimuli. The Journal of Neuroscience: The Official Journal of the Society for Neuroscience, 23(22), 8092-8097.

Zink, C. F., Pagnoni, G., Martin-Skurski, M. E., Chappelow, J. C., \& Berns, G. S. (2004). Human striatal responses to monetary reward depend on saliency. Neuron, 42(3), 509-517. 
Zink, C. F., Pagnoni, G., Chappelow, J., Martin-Skurski, M., \& Berns, G. S. (2006). Human striatal activation reflects degree of stimulus saliency. NeuroImage, 29(3), 977-983. https://doi.org/10.1016/j.neuroimage.2005.08.006 


\section{Acknowledgments}

First of all, I would like to thank Oliver Gruber, who gave me the opportunity to work on this topic. Thank you for your supervision, all the support and guidance while preparing, conducting and analyzing the present studies as well as your helpful comments and valuable suggestions.

Second, my gratitude goes to the members of my thesis committee Igor Kagan and Andreas Glöckner, for co-supervising this thesis and for the stimulating discussions during my $\mathrm{PhD}$. In addition, I would like to thank Peter Dechent, Roberto Goya-Maldonado and Annekathrin Schacht, who agreed to be part of my examination committee.

I am grateful that I had the opportunity to work with great people. I would like to thank my former and current colleagues for their assistance. Special thanks go to Bernd, Katja, Maria, Sarah T., Sarah W. and Sören, for sharing your professional expertise with me, for your help and discussions. Moreover, I thank you for helping me to immediately feeling home in Göttingen, sharing cookies, chocolate and all the lunch and funny short breaks. I am thankful for the creative and friendly working atmosphere and for your friendship even outside the lab.

My special gratitude goes to Roberto, for your support and encouragement and for your friendship.

I would also like to thank Ilona and Britta as well as the stuff from the MR-department, for their support during data acquisition, the cookies and conversations. I thank all the participants, who take part in my experiments and made the studies possible.

My gratitude goes to all my friends who accompanied me on my way. In particular, I would like to express my special thanks to Denise, for your substantial support and time, and to Katja G.: you were always there for me when it was necessary and helped me to see things through different eyes, thank you for that!

I wish to extend my wholehearted thanks to my dear parents, who always believed in me and supported as well as encouraged me on my way. Thank you for your trust and for always being there when I needed to talk.

Finally, my biggest gratitude goes to Benjamin, for your unshakeable belief in me, for supporting my decisions, enlightening my days, making me laugh whenever it was necessary - Thank you for all! 
